\title{
Fall prevention among people who have sustained an injurious fall : a multidisciplinary appraoch
}

Citation for published version (APA):

Bleijlevens, M. H. C. (2010). Fall prevention among people who have sustained an injurious fall : a multidisciplinary appraoch. [Doctoral Thesis, Maastricht University]. Datawyse / Universitaire Pers Maastricht. https://doi.org/10.26481/dis.20100416mb

Document status and date:

Published: 01/01/2010

DOI:

10.26481/dis.20100416mb

Document Version:

Publisher's PDF, also known as Version of record

\section{Please check the document version of this publication:}

- A submitted manuscript is the version of the article upon submission and before peer-review. There can be important differences between the submitted version and the official published version of record.

People interested in the research are advised to contact the author for the final version of the publication, or visit the DOI to the publisher's website.

- The final author version and the galley proof are versions of the publication after peer review.

- The final published version features the final layout of the paper including the volume, issue and page numbers.

Link to publication

\footnotetext{
General rights rights.

- You may freely distribute the URL identifying the publication in the public portal. please follow below link for the End User Agreement:

www.umlib.nl/taverne-license

Take down policy

If you believe that this document breaches copyright please contact us at:

repository@maastrichtuniversity.nl

providing details and we will investigate your claim.
}

Copyright and moral rights for the publications made accessible in the public portal are retained by the authors and/or other copyright owners and it is a condition of accessing publications that users recognise and abide by the legal requirements associated with these

- Users may download and print one copy of any publication from the public portal for the purpose of private study or research.

- You may not further distribute the material or use it for any profit-making activity or commercial gain

If the publication is distributed under the terms of Article $25 \mathrm{fa}$ of the Dutch Copyright Act, indicated by the "Taverne" license above, 
FALL PREVENTION AMONG PEOPLE WHO HAVE SUSTAINED AN INJURIOUS FALL:

\section{A MULTIDISCIPLINARY APPROACH}


The studies presented in this thesis were performed at the School for Public Health and Primary Care (Caphri) of the Maastricht University. Caphri participates in the Netherlands School of Primary Care Research (CaRe), re-acknowledged by the Royal Netherlands Academy of Arts and Sciences (KNAW) in 2006.

(C) Copyright Michel HC Bleijlevens, Maastricht 2010

Universitaire Pers Maastricht

ISBN 978-90-5278-927-9

Email:m.bleijlevens@zw.unimaas.nl

Maastricht University, Faculty of Health, Medicine and Life Sciences, Department of Health Care and Nursing Science,

School for Public Health and Primary Care (Caphri), P.O.Box 616, 6200 MD, Maastricht, The Netherlands

Cover design and lay-out:

Oburon Design (Robert Nijboer), www.oburondesign.nl

Printed by:

Datawyse bv / Universitaire Pers Maastricht 


\title{
FALL PREVENTION AMONG PEOPLE WHO HAVE SUSTAINED AN INJURIOUS FALL: A MULTIDISCIPLINARY APPROACH
}

\author{
PROEFSCHRIFT \\ ter verkrijging van de graad van doctor \\ aan de Universiteit Maastricht, \\ op gezag van de Rector Magnificus, \\ Prof. mr. G.P.M.F. Mols, \\ volgens het besluit van het College van Decanen, \\ in het openbaar te verdedigen \\ op vrijdag 16 april 2010 om 12.00 uur
}

door

Michel Hubertus Conraad Bleijlevens

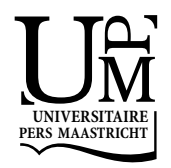




\section{Promotores}

Prof. dr. J.Th.M. van Eijk

Prof. dr. H.F.J.M. Crebolder

\section{Copromotor}

Dr. J.C.M. van Haastregt

\section{Beoordelingscommissie}

Prof. dr. G.J. Dinant (voorzitter)

Dr. J.F.B.M.H. Fiolet (azM)

Prof. dr. P.P. Geussens

Prof. dr. P.T.A.M. Lips (VUmc)

Prof. dr. J.M.G.A. Schols

ZonMw - The Netherlands Organization for Health Research and Development (grant number 945-02-053), the School for Public Health and Primary Care (Caphri), and Faculty of Health, Medicine and Life Sciences of the Maastricht University are gratefully acknowledged for funding the studies in this thesis. 
"OUD WORDEN IS EEN KUNST. SOMMIGE MENSEN HOUDEN VAN KUNST, ANDEREN NIET."

Bouwien Smits-Engelsman (Geriatrie in de fysiotherapeutische praktijk, 1999) 

CHAPTER 2: $\quad$ LACK OF EFFECTIVENESS OF A MULTIDISCIPLINARY FALL PREVENTION PROGRAMME IN ELDERLY PEOPLE AT RISK: A RANDOMISED CONTROLLED TRIAL [IRCTN64716113]

CHAPTER 3 : $\quad$ PROCESS FACTORS EXPLAINING THE INEFFECTIVENESS OF A MULTIDISCIPLINARY FALL PREVENTION PROGRAMME:

A PROCESS EVALUATION

CHAPTER 4 : LESSONS LEARNED FROM A MULTIDISCIPLINARY FALL PREVENTION PROGRAMME: THE OCCUPATIONAL-THERAPY PART

CHAPTER 5: INJURIOUS FALLS: RELATIONSHIP BETWEEN LOCATION AND ACTIVITY

CHAPTER 7 : GENERAL DISCUSSION

CHAPTER 8: $\quad$ SUMMARY

PUBLICATIONS 



\section{GENERAL INTRODUCTION}

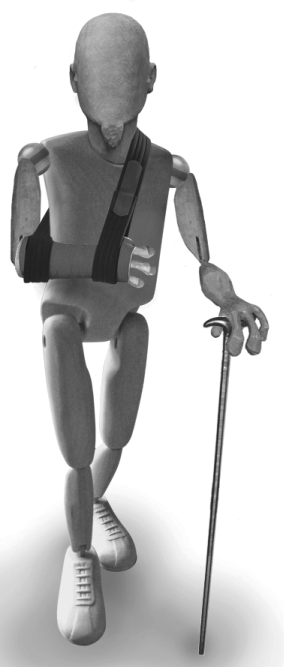




\section{INTRODUCTION}

This thesis describes the results of a study into the prevention of falls among older community-dwelling persons who have sustained an injurious fall. Several aspects of fall prevention among this group of persons are addressed, including effectiveness and feasibility of a multidisciplinary fall prevention programme, classification of injurious falls, and predictors of injurious falls.

In this chapter we describe the epidemiology and consequences of falls, the causes of and risk factors for falls, the prevention of falls, and the aims and outlines of the thesis.

\section{EPIDEMIOLOGY AND CONSEQUENCES OF FALLS}

As people get older the incidence of falls, as well as the severity of the consequences of falls, increases (1-6). In the last decades, several studies have shown that about one third of community-dwelling older people aged 65 years and over fall at least once each year $(3,5,7-12)$. The incidence of fallers who sustained two falls or more is approximately $15 \%(3,5,12-14)$. The falls rate among residents of nursing homes is even higher $(3,13-15)$. Fallers who have sustained a fall are more likely to sustain further falls $(3,8,11,16,17)$.

Falls severely threaten the health of older persons and can have a considerable impact on older persons and their relatives. Furthermore, falls place a heavy burden on healthcare systems in many countries (11). Although not all falls result in some kind of injury, in approximately $20 \%$ of falls medical attention is needed (18). In most cases falls result in minor injuries like lacerations without suture, bruises, sprains, abrasions, and other soft tissue injuries $(3,8,17,19-21)$. About $10 \%$ of the falls experienced by people aged 65 years and over result in major injuries comprising fractures, joint distortions and dislocations, severe head injuries, contusions, and lacerations requiring sutures $(2,5,6,8,11,18,20$, 22-24). Even if a fall does not result in any kind of physical injury, it can have a substantial impact on older people's psychosocial functioning. Several studies reported on psychosocial consequences of falls such as fear of falling and associated activity avoidance (5, 25-29). 
Together, physical and psychosocial consequences of a fall are responsible for an increase in functional limitations, deconditioning, reduced activities of daily living, decreased selfefficacy, and loss of self-confidence initiating a downward spiral (27, 30-34). Eventually, this may lead to increased dependency, increased immobility, and social isolation (27, 30-34). Furthermore, people who have sustained a fall show an increased morbidity and healthcare utilization, resulting in excessive healthcare costs, which are directly related to fall frequency and severity of injuries $(5,7,18,20,22,35-38)$. The largest share of the costs resulting from falls is due to hip fractures, mainly due to subsequent long-term admission to hospital or nursing home (39). Recent cost-of-illness studies confirm that falls are associated with substantial costs $(24,36,39-44)$.

\section{CAUSES AND RISK FACTORS FOR FALLS}

Unfortunately, falls are often regarded as a common, unavoidable, and untreatable consequence of aging. For this reason the focus of many healthcare workers is on the treatment of physical injuries from a fall instead of the prevention of falls (15). However, falls are often a sign or marker of an underlying acute or chronic medical problem and/or mobility impairment that can be amenable to treatment. Therefore, a fall should be viewed as a nonspecific event representative of an underlying problem attributed to specific risk factors $(15,45)$. Due to the multifactorial origin of falls, it is often not possible to determine a single cause for falling $(13,15)$. Therefore, many studies tried to identify risk factors for falls in order to select persons most at risk for falling $(5,8-11,13,17,18,21,22,32,46,47)$. However, it has been difficult to compare the outcomes of these studies due to a number of methodological issues, such as differences in study population, differences in definitions of a fall, differences in length of follow-up periods, differences in statistical techniques, and difficulties with retrospective recall of falls $(11,16,17)$.

Researchers have identified more than 400 possible risk-factors for falls (8). The large number of suggested possible risk factors for falls reflects the complexity of the aetiology of falls. In general, risk factors for falls can be classified into intrinsic and extrinsic risk factors $(11,15,22,46,48)$. Intrinsic risk factors are related to the individual such as demographic characteristics (age, gender, marital status, ethnicity, education, socio-economic status, etc.). 
They also comprises medical conditions, mobility impairments (including muscle weakness, gait disorders, and impaired balance), nutritional deficiencies, visual impairment, foot problems, impaired cognition, psychological status (fear of falling), use of psychotropic drugs, history of falls, and sedentary behaviour (3, 5, 10, 11, 13, 14, 17, 19, 49-51). Extrinsic risk factors refer to situational or environmental risk factors like uneven or slippery floor surfaces, inadequate lighting, loose rugs, and unstable furniture. In addition, fall conditions can be caused by individual belongings worn and/or used by a person like the type of footwear (socks, slippers, shoes, etc) and inappropriate use of walking aids and assistive devices $(11,52)$. The impact of extrinsic risk factors on falls is still doubtful. No convincingly causal relationship between falls and extrinsic risk factors has been found. The presence of extrinsic risk factors alone is not enough to cause falls $(38,52-54)$. It is recognised more and more that falls are the consequence of the interaction between a number of risk factors, both intrinsic and extrinsic $(3,5,14,19,52)$. An extrinsic risk factor may provoke a fall. However, whether or not a person will fall also depends on intrinsic factors. Fall risk increases with the presence of more risk factors $(5,50)$.

\section{FALL PREVENTION STRATEGIES}

In view of the impact and consequences of falls, it is important to develop fall prevention strategies. Developing effective fall prevention programmes requires particular knowledge of risk factors. Several risk factors for falls can be influenced and are amenable to improvement (16-18, 32). During the last decade many successful fall prevention programmes have been developed, mostly based on the identification and treatment of risk factors $(1,18,22$, $55,56)$. In general, fall prevention programmes comprise two different approaches: singleintervention strategies (like strength and balance training, intake of nutritional supplements, and reduction in psychotropic drugs usage) on the one hand and multifactorial strategies, aimed at more than one risk factor simultaneously, on the other hand $(1,18,22)$. Fall prevention strategies need to demonstrate (cost-) effectiveness and feasibility for both participants and providers.

At the time of the start of the study described in the current thesis, fall prevention had been a focus of interest for years and as stated above, many programmes aimed at preventing falls 
have been developed and evaluated. Although many of these turned out to be ineffective, there was considerable evidence for the effectiveness of multidisciplinary and multifactorial interventions (57-59). Multifactorial and multidisciplinary fall prevention programmes screen for health and environmental risk factors and offer interventions, both for the general population of community-dwelling elderly people and for community-dwelling elderly people with a history of falling who are selected because of known risk factors (22). In particular, these programmes seem to be most effective when tailor made and aimed at high-risk populations such as community-dwelling elderly people with a history of (injurious) falls, or who are suffering from mobility impairments $(1,11,60)$. One such multi-faceted programme to prevent falls among elderly people attending the Accident and Emergency (A\&E) department after an injurious fall is the multidisciplinary programme developed by Close and colleagues (58). This programme has been evaluated in a randomised controlled trial (PROFET) in the United Kingdom (UK) and had favourable effects on falls and daily functioning (58). The PROFET study demonstrated that a multidisciplinary intervention programme comprising a medical and occupational-therapy assessment implemented among people at risk was highly effective in reducing the number of recurrent falls and associated injuries in London (United Kingdom) (58). Since characteristics of the participants and healthcare setting appear to be critical, it cannot be automatically assumed that when a fall prevention programme is effective in a specific healthcare setting, this will be also the case in another healthcare setting (22). We therefore adapted the programme developed by Close and colleagues (58) to the Dutch situation, and made adjustments based on recent insights in the literature and recommendations from experts in the care of older persons. The adapted version of the programme was tested in a pilot study $(n=36)(61)$. The final programme included a medical and occupational therapy assessment resulting in recommendations and/or further referrals if indicated. The medical assessment comprised an examination performed by a geriatrician, a geriatric nurse, and a rehabilitation physician to identify and address risk factors for falling. The examination included a comprehensive general examination, but in addition focused on a more detailed assessment of visual acuity, stereoscopic vision, mobility, balance, cognition, affect, use of medication, and examination of feet and footwear. Recommendations or indications for referral resulting from this examination were sent to the patient's general practitioner (GP). Patients were advised to contact their GPs to be informed of the results of the medical assessment and the recommendations and/or referrals resulting from it. After the medical assessments, an occupational therapist visited the patients 
to identify possible risk factors for falling in the home environment. The therapist made recommendations regarding adaptations to the home environment, assistive devices, and behavioural change. Recommendations by the occupational therapist were sent directly to the patients themselves and to their GPs (61-64). After adapting the programme developed by Close and colleagues (58) to the Dutch situation we evaluated the effectiveness of the adapted programme in a randomised controlled trial (RCT) in the Netherlands $(61,64)$.

\section{AIMS AND OUTLINES OF THE THESIS}

\section{Aims}

This thesis explores the effectiveness and feasibility of a medical and occupational-therapy programme to prevent falls and functional decline in elderly people at risk compared to usual healthcare in the Netherlands. In addition it explores opportunities to improve the prevention of injurious falls. The aims of this study are:

1. To assess the effectiveness of a multifactorial medical and occupational-therapy fall prevention programme among elderly people at risk, in terms of falls, functional decline, and a number of secondary outcomes.

2. To assess the feasibility of this fall prevention programme for elderly people as well as the medical and paramedical practitioners who performed the assessments.

3. To assess the role of the occupational-therapy part of the fall prevention programme in preventing new falls and functional decline.

4. To assess whether it is possible to establish a classification of injurious fall types based on fall location and activity up to the moment of the fall.

5. To assess which risk factors predict new falls among a group of community-dwelling injurious fallers aged 65 years or over, in order to achieve a better selection of older people at high risk for falling.

\section{Outline}

Chapter 2 presents the results of an RCT which tested the effectiveness of a multidisciplinary fall prevention programme aimed at people aged 65 years and over living in the community who had visited the A\&E department due to the consequences of a fall. The feasibility of the multifactorial fall prevention programme is studied in Chapter 3 . This chapter reports 
on the results of the process evaluation and focuses mainly on the medical part of the intervention programme. Subsequently, Chapter 4 reports on the results of an in-depth analysis of the occupational-therapy part of the intervention programme, in order to gain insight into the contribution of the occupational-therapy programme towards the reduction in falls and functional decline. Chapter 5 reports on the relationship between location of the fall and activity up to the moment of the fall resulting in a classification of injurious falls. Chapter 6 reports a study assessing which risk factors predict new falls among a group of community-dwelling injurious fallers aged 65 years or over. Based on the outcomes of this study we developed a feasible fall risk screening tool for the healthcare professionals involved in fall prevention. Chapter 7 will discuss the main findings and some theoretical and methodological considerations of the studies presented in this thesis, and will conclude with some implications for clinical practice as well as implications for future research. 


\section{REFERENCES}

1. Chang JT, Morton SC, Rubenstein LZ, Mojica WA, Maglione M, Suttorp MJ, et al. Interventions for the prevention of falls in older adults: systematic review and meta-analysis of randomised clinical trials. BMJ. 2004;328(7441):680.

2. Kannus P, Parkkari J, Koskinen S, Niemi S, Palvanen M, Jarvinen M, et al. Fall-induced injuries and deaths among older adults. JAMA. 1999 May 26;281(20):1895-9.

3. O'Loughlin J, Robitaille Y, Bolvin J-F, Suissa S. Incidence of and risk factors for falls and injurious falls among the community-dwelling elderly. Am J Epidemiol. 1993;137:342-54.

4. Rubenstein LZ, Josephson KR, Robbins AS. Falls in the nursing home. Ann Intern Med. 1994 Sep $15 ; 121(6): 442-51$.

5. Tinetti ME, Speechley M, Ginter SF. Risk factors for falls among elderly persons living in the community. N Engl J Med. 1988;319(26):1701-7.

6. van Weel C, Vermeulen H, van den Bosch W. Falls, a community care perspective. Lancet. 1995 Jun 17;345(8964): 1549-51.

7. Blake AJ, Morgan K, Bendall MJ, Dallosso H, Ebrahim SB, Arie TH, et al. Falls by elderly people at home: prevalence and associated factors. Age Ageing. 1988;17(6):365-72.

8. Masud T, Morris, R.O. Epidemiology of falls. Age Ageing. 2001;30 (S4):3-7.

9. Stalenhoef PA, Diederiks JP, Knottnerus JA, Kester AD, Crebolder HF. A risk model for the prediction of recurrent falls in community-dwelling elderly: a prospective cohort study. J Clin Epidemiol. 2002 Nov;55(11): 1088-94.

10. Tromp AM, Pluijm SM, Smit JH, Deeg DJ, Bouter LM, Lips P. Fall-risk screening test: a prospective study on predictors for falls in community-dwelling elderly. J Clin Epidemiol. 2001 Aug;54(8):837-44.

11. Todd C, Skelton D. What are the main risk factors for falls among older people and what are the most effective interventions to prevent falls? Copenhagen: WHO Regional Office for Europe (Health Evidence Network report); 2004 March 2004. Report No.: E82552.

12. Luukinen H, Koski K, Hiltunen L, Kivela SL. Incidence rate of falls in an aged population in northern Finland. J Clin Epidemiol. 1994 Aug;47(8):843-50.

13. Campbell AJ, Borrie MJ, Spears GF. Risk factors for falls in a community-based prospective study of people 70 years and older. J Gerontol. 1989;44(4):M112-7.

14. Nevitt MC, Cummings, S.R., Kidd, S., Black, D. Risk factor for recurrent nonsyncopal falls. JAMA. 1989;261:2663-8.

15. Bloem BR, Boers, I., Cramer, M., Westendorp, R.G.J., Gerschlager, W. Falls in the elderly: identification of risk factors. Wiener Klinische Wochenschrift. 2001;113:352-62. 
16. Russell MA, Hill KD, Blackberry I, Day LL, Dharmage SC. Falls risk and functional decline in older fallers discharged directly from emergency departments. J Gerontol A Biol Sci Med Sci. 2006 Oct;61(10):1090-5.

17. Stalenhoef P, Crebolder HF, Knottnerus A, F. Hvd. Incidence, risk factors and consequences of falls among elderly subject living in the community. Eur J Pub Health. 1997;7:328-34.

18. Kannus $P$, Sievanen $H$, Palvanen $M$, Jarvinen $T$, Parkkari J. Prevention of falls and consequent injuries in elderly people. Lancet. 2005 Nov 26;366(9500):1885-93.

19. Campbell AJ, Borrie MJ, Spears GF, Jackson SL, Brown JS, Fitzgerald JL. Circumstances and consequences of falls experienced by a community population 70 years and over during a prospective study. Age Ageing. 1990;19(2):136-41.

20. Nevitt MC, Cummings SR, Hudes ES. Risk factors for injurious falls: a prospective study. J Gerontol. 1991;46(5):M164-70.

21. Tromp AM, Smit JH, Deeg DJ, Bouter LM, Lips P. Predictors for falls and fractures in the Longitudinal Aging Study Amsterdam. J Bone Miner Res. 1998 Dec;13(12):1932-9.

22. Gillespie LD, Gillespie WJ, Robertson MC, Lamb SE, Cumming RG, Rowe BH. Interventions for preventing falls in elderly people. Cochrane Database Syst Rev. 2003(4):CD000340.

23. McClure R, Turner C, Peel N, Spinks A, Eakin E, Hughes K. Population-based interventions for the prevention of fall-related injuries in older people. Cochrane Database Syst Rev. 2005(1):CD004441.

24. Carroll NV, Slattum PW, Cox FM. The cost of falls among the community-dwelling elderly. J Manag Care Pharm. 2005 May; 11(4):307-16.

25. Friedman SM, Munoz B, West SK, Rubin GS, Fried LP. Falls and fear of falling: which comes first? A longitudinal prediction model suggests strategies for primary and secondary prevention. J Am Geriatr Soc. 2002 Aug;50(8):1329-35.

26. Tinetti ME, Mendes de Leon CF, Doucette JT, Baker DI. Fear of falling and fall-related efficacy in relationship to functioning among community-living elders. J Gerontol. 1994 May;49(3):M140-7.

27. Vellas BJ, Wayne SJ, Romero LJ, Baumgartner RN, Garry PJ. Fear of falling and restriction of mobility in elderly fallers. Age Ageing. 1997;26:189-93.

28. Howland J, Peterson EW, Levin WC, Fried L, Pordon D, Bak S. Fear of falling among the communitydwelling elderly. J Aging Health. 1993 May;5(2):229-43.

29. Wilson MM, Miller DK, Andresen EM, Malmstrom TK, Miller JP, Wolinsky FD. Fear of falling and related activity restriction among middle-aged African Americans. J Gerontol A Biol Sci Med Sci. 2005 Mar;60(3):355-60.

30. Jorstad EC, Hauer K, Becker C, Lamb SE. Measuring the psychological outcomes of falling: a systematic review. J Am Geriatr Soc. 2005 Mar;53(3):501-10. 
31. Kannus P, Khan KM. Prevention of falls and subsequent injuries in elderly people: a long way to go in both research and practice. CMAJ. 2001;165(5):587-88.

32. Rubenstein LZ. Falls in older people: epidemiology, risk factors and strategies for prevention. Age Ageing. 2006 Sep;35 Suppl 2:ii37-ii41.

33. Vellas B, Cayla F, Bocquet $H$, de Pemille F, Albarede JL. Prospective study of restriction of activity in old people after falls. Age Ageing. 1987 May; 16(3):189-93.

34. ZijJstra GA, van Haastregt JC, van Eijk JT, van Rossum E, Stalenhoef PA, Kempen GI. Prevalence and correlates of fear of falling, and associated avoidance of activity in the general population of communityliving older people. Age Ageing. 2007 May;36(3):304-9.

35. Kiel DP, O'Sullivan P, Teno JM, Mor V. Health care utilization and functional status in the aged following a fall. Med Care. 1991;29(3):221-8.

36. Meerding WJ, Mulder S, van Beeck EF. Incidence and costs of injuries in The Netherlands. Eur J Public Health. 2006 Jun;16(3):272-8.

37. Rizzo JA, Friedkin R, Williams CS, Nabors J, Acampora D, Tinetti ME. Health care utilization and costs in a Medicare population by fall status. Med Care. 1998 Aug;36(8):1174-88.

38. Sattin RW, Rodriguez JG, DeVito CA, Wingo PA. Home environmental hazards and the risk of fall injury events among community-dwelling older persons. J Am Geriatr Soc. 1998;46:669-76.

39. Polder JJ, Takken J, Meerding WJ, Kommer GJ, Stokx LJ. Kosten van Ziekten in Nederland. De zorgeuro ontrafeld. Bilthoven: Rijksinstituut voor Volksgezondheid en Milieu; 2002. Report No.: RIVM rapport 270751005.

40. Meerding WJ, Bonneux L, Polder JJ, Koopmanschap MA, van der Maas PJ. Demographic and epidemiological determinants of healthcare costs in Netherlands: cost of illness study. BMJ. $1998 \mathrm{Jul}$ 11;317(7151):111-5.

41. Newton JL, Kyle P, Liversidge P, Robinson G, Wilton K, Reeve $P$. The costs of falls in the community to the North East Ambulance Service. Emerg Med J. 2006 Jun;23(6):479-81.

42. Roudsari BS, Ebel BE, Corso PS, Molinari NA, Koepsell TD. The acute medical care costs of fall-related injuries among the U.S. older adults. Injury. 2005 Nov;36(11):1316-22.

43. Scuffham P, Chaplin S, Legood R. Incidence and costs of unintentional falls in older people in the United Kingdom. J Epidemiol Community Health. 2003 Sep;57(9):740-4.

44. Stevens JA, Corso PS, Finkelstein EA, Miller TR. The costs of fatal and non-fatal falls among older adults. Inj Prev. 2006 Oct; 12(5):290-5.

45. Rubenstein LZ, Josephson KR. Falls and their prevention in elderly people: what does the evidence show? Med Clin North Am. 2006 Sep; 90(5):807-24. 
46. Cesari M, Landi F, Torre S, Onder G, Lattanzio F, Bernabei R. Prevalence and risk factors for falls in an older community-dwelling population. J Gerontol A Biol Sci Med Sci. 2002 Nov;57(11):M722-6.

47. Stel VS, Pluijm SM, Deeg DJ, Smit JH, Bouter LM, Lips P. A classification tree for predicting recurrent falling in community-dwelling older persons. J Am Geriatr Soc. 2003 Oct;51(10):1356-64.

48. American Geriatrics Society BGS, and American Academy of Orthopaedic Surgeons Panel on Falls Prevention. Guideline for the prevention of falls in older persons. J Am Geriatr Soc. 2001;49(5):664-72.

49. Bueno Cavanillas A, Padilla Ruiz F, Jimenez Moleon JJ, Peinado Alonso CA, Galvez Vargas R. Risk factors in falls among the elderly according to extrinsic and intrinsic precipitating causes. Eur J Epidemiol. 2000;16(9):849-59.

50. Graafmans WC, Ooms ME, Hofstee HM, Bezemer PD, Bouter LM, Lips P. Falls in the elderly: a prospective study of risk factors and risk profiles. Am J Epidemiol. 1996;143(11):1129-36.

51. Cozart HC, Cesario SK. Falls aren't us: state of the science. Crit Care Nurs Q. 2009 Apr-Jun;32(2):116-27.

52. Lord SR, Menz HB, Sherrington C. Home environment risk factors for falls in older people and the efficacy of home modifications. Age Ageing. 2006 Sep;35 Suppl 2:ii55-ii9.

53. Carter SE, Campbell EM, Sanson-Fisher RW, Redman S, Gillespie WJ. Environmental hazards in the homes of older people. Age Ageing. 1997;26:195-202.

54. Stalenhoef P, Diederiks J, Knottnerus A, de Witte L, Crebolder H. How predictive is a home-safety checklist for indoor fall risk in the elderly living in the community? Eur J Gen Pract. 1998;4:114-20.

55. Gates S, Lamb SE, Fisher JD, Cooke MW, Carter YH. Multifactorial assessment and targeted intervention for preventing falls and injuries among older people in community and emergency care settings: systematic review and meta-analysis. BMJ. 2008 Jan 19;336(7636):130-3.

56. Gillespie L. Preventing falls in elderly people. BMJ. 2004 Mar 20;328(7441):653-4.

57. Guideline for the prevention of falls in older persons. American Geriatrics Society, British Geriatrics Society, and American Academy of Orthopaedic Surgeons Panel on Falls Prevention. J Am Geriatr Soc. 2001 May;49(5):664-72.

58. Close J, Ellis M, Hooper R, Glucksman E, Jackson S, Swift C. Prevention of falls in the elderly trial (PROFET): a randomised controlled trial. Lancet. 1999 Jan 9;353(9147):93-7.

59. Feder G, Cryer C, Donovan S, Carter Y. Guidelines for the prevention of falls in people over 65. The Guidelines' Development Group. BMJ. 2000 Oct 21;321(7267):1007-11.

60. Ganz DA, Bao Y, Shekelle PG, Rubenstein LZ. Will my patient fall? JAMA. 2007 Jan 3;297(1):77-86.

61. Hendriks MR, Bleijlevens MH, van Haastregt JC, de Bruijn FH, Diederiks JP, Mulder WJ, et al. A multidisciplinary fall prevention program for elderly persons: a feasibility study. Geriatr Nurs. 2008 MayJun;29(3): 186-96. 
62. Bleijlevens MH, Hendriks MR, van Haastregt JC, van Rossum E, Kempen GI, Diederiks JP, et al. Process factors explaining the ineffectiveness of a multidisciplinary fall prevention programme: a process evaluation. BMC Public Health. 2008 Sep 24;8(1):332.

63. Hendriks MR, Bleijlevens MH, van Haastregt JC, Crebolder HF, Diederiks JP, Evers SM, et al. Lack of effectiveness of a multidisciplinary fall-prevention program in elderly people at risk: a randomized, controlled trial. J Am Geriatr Soc. 2008 Aug;56(8):1390-7.

64. Hendriks MR, van Haastregt JC, Diederiks JP, Evers SM, Crebolder HF, van Eijk JT. Effectiveness and costeffectiveness of a multidisciplinary intervention programme to prevent new falls and functional decline among elderly persons at risk: design of a replicated randomised controlled trial [ISRCTN64716113]. BMC Public Health. 2005 Jan 14;5:6. 


\title{
LACK OF EFFECTIVENESS OF A MULTIDISCIPLINARY
}

\author{
FALL PREVENTION PROGRAMME IN ELDERLY \\ PEOPLE AT RISK: A RANDOMISED CONTROLLED \\ TRIAL [IRCTN64716113]
}

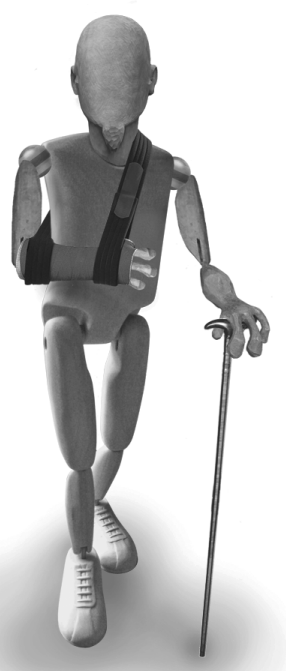

\author{
Marike RC Hendriks \\ Michel HC Bleijlevens \\ Jolanda CM van Haastregt \\ Harry FJM Crebolder \\ Joseph PM Diederiks \\ Silvia MAA Evers \\ Gertrudis IJM Kempen \\ Erik van Rossum \\ Joop M Ruijgrok \\ Paul A Stalenhoef \\ Wubbo J Mulder \\ Jacques ThM van Eijk
}

Journal of the American Geriatrics Society 2008; 56(8):1390-7 


\section{ABSTRACT}

Objective To assess whether a pragmatic multidisciplinary fall prevention programme was more effective than usual care in preventing new falls and functional decline among elderly people.

Design A two-group randomised controlled trial with 12 months of follow-up.

Setting University hospital and home-based intervention, the Netherlands.

\section{Participants}

Three hundred thirty-three community-dwelling Dutch people aged 65 years or over who were seen in an accident and emergency department after a fall.

\section{Intervention}

Participants in the intervention group underwent a detailed medical and occupational-therapy assessment to evaluate and address risk factors for recurrent falls, followed by recommendations and referral if indicated. People in the control group received usual care.

\section{Measurements}

Number of people sustaining a fall (fall calendar) and daily functioning (Frenchay Activity Index).

\section{Results}

Results showed no statistically significant favourable effects on falls (Odds Ratio $=0.86,95 \%$ Confidence Interval $(\mathrm{Cl})=0.50$ to 1.49 ) or daily functioning (Regression Coefficient $=0.37, \mathrm{Cl}=$ -0.90 to 1.63 ) after twelve months of follow-up.

\section{Conclusion}

The multidisciplinary fall prevention programme is not effective in preventing falls and functional decline in this Dutch healthcare setting. Implementing the programme in its present form in the Netherlands is not recommended. This trial shows that there can be considerable discrepancy between the "ideal" (experimental) version of a programme and the implemented version of the same programme. The importance of implementation research in assessing feasibility and effectiveness of such a programme in a specific healthcare setting is therefore stressed. 


\section{INTRODUCTION}

Falls are common occurrences among elderly people worldwide and may have several adverse consequences, such as physical injuries and psychological distress, leading to decreased functioning and quality of life $(1,2)$. Injurious falls in particular are associated with an increase in healthcare utilization (3). The need for effective fall prevention strategies is thus evident. There is now considerable evidence for the effectiveness of multidisciplinary and multifactorial interventions that screen for health and environmental risk factors and address these factors $(1,4,5)$. A medical and occupational-therapy (OT) assessment for elderly persons attending an accident and emergency department in the inner city of London (United Kingdom) after an injurious fall was developed. This programme showed convincing favourable effects on falls and daily functioning, but when a programme has been shown to be effective in an experimental setting, it is important to assess whether it is also effective when implemented as a part of routine healthcare. A version of this programme, adapted to the Dutch heath system, was therefore evaluated to assess its robustness and to ascertain whether it could be recommended for implementation in the Netherlands.

The objective of the current study was to assess whether a multidisciplinary fall prevention programme was more effective than usual care in preventing new falls and functional decline in elderly community-dwelling Dutch people who attended an emergency department (ED) after a fall. A fall was defined as an event that results in a person coming to rest inadvertently on the ground or other lower level (6), and an injurious fall as a fall for which medical care is sought.

\section{METHODS}

\section{Study design and participants}

The study design was a two-group randomised controlled trial with 12 months of follow-up. Outcomes were measured at four and twelve months after baseline. Sample size calculations indicated that 164 persons per group had to be included to detect the same reduction in falls as was found in a previous study ( $52 \%$ fallers in the usual care group and $32 \%$ fallers in the intervention group), with a power of $90 \%$, an alpha of 0.05 , and an expected dropout rate of 
$25 \%$ during 12 months of follow-up $(2,7)$. The current study included community-dwelling people aged 65 years and over who attended ED of the University Hospital Maastricht for the consequences of a fall. Because the General Practitioner's Cooperative (GP Cooperative) provides after-hours emergency care in Maastricht (8), participants were also recruited at the GP Cooperative. People were excluded if they were unable to speak Dutch, were cognitively impaired (a score $<4$ on the Abbreviated Mental Test 4) (9), had been admitted for more than 4 weeks to a hospital or another institution, and/or were permanently wheelchair-dependent or bedridden. An external agency allocated eligible participants who signed the informed consent form and returned a completed baseline questionnaire to the intervention or control group according to computerised alternate random allocation. Participants allocated to the intervention group underwent medical and OT assessments, followed by recommendations or further referral if indicated. The control group received usual care only. No restrictions were placed on co-interventions. To ensure blinding during data collection, measurements by phone were contracted to an independent call centre (Centre for Data and Information Management, MEMIC), whose operators were unaware of group allocation. The Medical Ethics Committee of the Maastricht University and University Hospital approved the design, which is described in detail elsewhere (7).

\section{Intervention}

\section{Fall prevention programme}

A programme develop previously (2) was adapted to make it implementable in the Dutch setting, and its feasibility was subsequently assessed in a pilot study. The feasibility study revealed that some adaptations programme adaptations were necessary to increase the chance of successful implementation in the Netherlands. The adaptation process and feasibility study are described elsewhere (10). Briefly, the adapted programme consisted of structured medical and OT assessments to assess and address potential risk factors for new falls. The medical and OT assessments were described in a protocol. The medical assessments were performed at the Maastricht University Hospital and comprised a comprehensive general examination, and a more detailed assessment of vision, $(11,12)$ sense of hearing, locomotor apparatus, (13) feet and footwear, (14) peripheral nervous system, balance and mobility (Romberg and Get Up and Go Test), 


\section{Table 1 - Medical and occupational-therapy assessments}

\begin{tabular}{|c|c|}
\hline \multicolumn{2}{|l|}{ Medical Assessment } \\
\hline Assessments & Details/Tools/Test Batteries \\
\hline Standard examination & $\begin{array}{l}\text { Anamnesis and fall history } \\
\text { Cardiovascular, respiratory, abdominal } \\
\text { system and neurological system }\end{array}$ \\
\hline Blood pressure & Supine and erect \\
\hline Vision & $\begin{array}{l}\text { Visual acuity (Snellen) (11) } \\
\text { Visual fields (confrontation) } \\
\text { Stereoscopic vision (12) }\end{array}$ \\
\hline Sense of hearing & Whispered voice test \\
\hline $\begin{array}{l}\text { Locomotor apparatus: lumbar spine, upper } \\
\text { and lower extremities }\end{array}$ & $\begin{array}{l}\text { Tone \& Power (MRC Scale) (13) } \\
\text { Joint deformity } \\
\text { Range of movement } \\
\text { Handgrip dynamometry }\end{array}$ \\
\hline Feet and footwear & $\begin{array}{l}\text { Callus, skin ulcers, oedema and arterial } \\
\text { pulsations } \\
\text { Footwear assessment form (14) }\end{array}$ \\
\hline Peripheral nervous system & $\begin{array}{l}\text { Sensation (monofilaments) } \\
\text { Vibration (tuning fork) } \\
\text { Proprioception (great toe) }\end{array}$ \\
\hline Balance and mobility & $\begin{array}{l}\text { Romberg (15) } \\
\text { Get Up and Go Test (16) }\end{array}$ \\
\hline Anthropometric indices & $\begin{array}{l}\text { Body weight (kg) } \\
\text { Height }(\mathrm{m})\end{array}$ \\
\hline Cognition & Mini Mental State Examination (17) \\
\hline Affect & Geriatric Depression Scale (18) \\
\hline Heart & Electrocardiogram \\
\hline Blood tests & If indicated \\
\hline Medication & Prescribed medication list \\
\hline \multicolumn{2}{|l|}{ Occupational Therapy Assessment } \\
\hline Function assessment & Frenchay Activity Index (20) \\
\hline & $\begin{array}{l}\text { Checklist of the local agency deciding on } \\
\text { eligibility for care, aids and devices }(21,22)\end{array}$ \\
\hline Environmental hazards & Dutch version of the Home Checklist (23) \\
\hline Psychological consequences of the fall & Falls Handicap Inventory (24) \\
\hline
\end{tabular}


$(15,16)$ anthropometry, cognition (Mini Mental State Examination), (17) affect (Geriatric Depression Scale), (18) blood tests if indicated and medication use. Table 1 presents details about the assessments.

A geriatrician working in a day hospital who was at the same time the main researcher of the trial evaluating the programme performed the medical assessment in the British programme. In the British setting, the geriatrician could directly refer patients to other relevant disciplines. In the Netherlands, this approach would not fit in with regular care, because the geriatrician is not a member of the ED staff and nonacute referrals to medical specialists have to be made through the participants' GP. Otherwise health insurance companies will not cover the costs of these referrals) (19). In addition, GPs are familiar with the health status of their patients and can act as supervisors to provide the best possible care. It was therefore decided to incorporate the GPs in the programme by sending them a summary of the results of the medical assessments, written by the geriatrician. If necessary, this summary also included recommendations and/or referrals to relevant services. Participants were recommended to contact their GP about these results, recommendations, and/or referrals following the medical assessment. The GPs could then take action if they agreed with the recommendations and/ or referrals. Because geriatric nurses usually assist the geriatrician in the Netherlands, a geriatric nurse was also incorporated to perform the less-complex assessments. In addition, a rehabilitation physician was included, because rehabilitation physicians are more specialised in examining feet and footwear (14) than geriatricians.

After the medical assessment, an occupational therapist visited the participants at home for a structured functional and environmental assessment. Daily functioning was assessed using the 15-item Frenchay Activity Index (FAI) (20) and an OT checklist (21, 22). Environmental hazards were identified and registered by means of a home-safety checklist (23). In addition, the Falls Handicap Inventory (FHI) (24) was used to assess handicaps associated with repeated falls. The participants received recommendations with regard to behavioural change, functional needs and safety within the home environment. Technical aids and adaptations or additional support were directly referred to and delivered by social and community services. To increase compliance, participants were sent a letter with the recommendations and/or referrals from the occupational therapist, by way of reminder. A copy of this letter was sent to the participants' GPs. 
The medical assessment was scheduled to take place in the first month after baseline. Subsequently, the home assessment was scheduled within 1 month after the medical assessment. Afterwards, a summary of the results and recommendations for further referral were sent to the participants' GP. Therefore, it was scheduled to take at approximately 2.5 months (with a maximum 3.5 months) after baseline measurement for all recommendations to reach the participants and be implemented.

\section{Usual care}

Currently, no standard approach to fall risk assessment is available for fallers presenting to the ED and being discharged home (25). In usual care in the Netherlands hospital physicians, specialists and GPs do not systematically record or address medical risks and other risk factors for falls, such as environmental hazards in the home and patients' risk behaviour. Moreover, when people present to the ED or the GP cooperative, no systematic attention is usually given to the specific consequences of injurious falls for the daily functioning of individual patients in their unique situation.

\section{Measurements}

The primary outcome measures were falls (i.e. falls, recurrent falls, injurious falls and time to first fall) and daily functioning. Falls were measured as the percentage of participants sustaining at least one fall during the follow-up, recurrent falls as the percentage of participants sustaining two or more falls during follow-up and injurious falls as the percentage of participants who sought medical care after a fall. Time to the first fall was measured in weeks. Participants recorded their falls continuously on a fall calendar for 12 months after baseline. MEMIC contacted them monthly by telephone to collect information on the falls noted on the calendar. Daily functioning was measured using the FAI (20) in a selfadministered questionnaire at baseline and after 4 and 12 months.

Secondary outcome measures were: recuperation from the index fall (1 item), health complaints (19 items), perceived health (first item of the RAND-36) (26), activities of daily living ( $A D L$ ) and instrumental ADL disability (Groningen Activity Restriction Scale) (27), mental health (Hospital Anxiety and Depression Scale) (28), fear of falling (1 item), activity 
avoidance (1 item), social participation (2 items), and quality of life (EuroQol converted into utilities according to Dolans' tariffs) $(29,30)$. These outcomes were also measured using self-administered questionnaires at baseline and after 4 and 12 months.

At baseline, the following background characteristics were assessed: age, gender, living situation (living alone vs not living alone), level of education (primary school or less vs more than primary school), self-reported weight, type of injury resulting from the index fall (fracture or joint dislocation, i.e. major injury, vs minor injury), handicaps associated with the index fall (Falls Handicap Inventory) (24), and number of illnesses (20 items).

\section{Analysis}

SPSS statistical software (version 13) was used for the analyses (SPSS Inc., Chicago, IL). Baseline characteristics of the intervention and control groups were analysed using descriptive statistics. Short-term and long-term effects were analysed according to the intention-to-treat principle, including all participants with valid data, regardless of whether they received the programme. Differences in outcomes between the groups were analysed using multiple linear and logistic regressions and were adjusted for potential differences in covariates measured at baseline (i.e. the relevant outcome measure, age, gender, living situation, education, injury from the index fall, weight, illnesses, recurrent falls, and psychological consequences of the index fall). Survival analysis using Cox Proportional Hazards regression was used to study the time to first fall for each participant. In addition, per protocol analyses were performed on primary outcomes (at 12 months) for participants in the intervention group who received the complete medical and OT assessments. Subgroup analyses were performed with groups considered to be at higher fall risk $(31,32)$ (people with a history of $>1$ fall in the previous year, people with mobility impairments (defined as reporting some problems with walking, or worse, on the mobility item of the EuroQol) (29), poor ADL functioning (defined as a score $<30$ on the FAI) (20), and older age (80 years and older)). Per-protocol and subgroup analyses were adjusted for the same covariates as the intention-to-treat analysis.

Analyses of falls and recurrent falls included persons for whom at least 9 of the 12 months of the fall calendar data were available. Missing data were replaced using the individual 
mean of valid data. Injurious falls were analysed by means of complete case analysis, because imputation of the data would lead to underreporting of injurious falls. In addition, a sensitivity analysis was performed to determine whether analyses with those participants with complete data on all 12 fall calendar months (complete case analyses) would result in other conclusions about effectiveness.

\section{RESULTS}

\section{Participants}

A detailed overview of the progress of participants during the trial is shown in figure 1 . From January 2003 until March 2004, 2362 persons were recruited after they had attended the ED and/or GP cooperative because of a fall. Thirty-eight percent did not meet the inclusion criteria $(n=774)$ and $26 \%$ refused to participate (e.g., not interested, too time consuming; $\mathrm{n}=531$ ). Finally, 333 participants gave informed consent and were randomly allocated to the intervention $(n=166)$ or control group $(n=167)$. After 12 months of follow-up, $25 \%$ ( $n=42)$ of the participants had dropped out of the intervention group, and $20 \%(n=33)$ out of the control group. The main reason for dropping out of the study was health problems. Reasons for dropout were similar in both groups.

Table 2 shows the baseline characteristics of the study population. Randomization achieved approximately equal balance between the groups.

The 75 participants who withdrew from the study during the 12-month follow-up period were on average older (77.2 vs 74.2 years, $p=0.000$ ), had lower scores on the FAI at baseline (people who withdrew being more inactive, 20.8 vs 24.2, $p=0.002$ ), and had higher scores on the $\mathrm{FHI}$ at baseline (participants who withdrew reporting more impairments associated with repeated falls, 30.7 vs 20.2, $p=0.000$ ). In addition, participants who withdrew reported more illnesses at baseline than those who completed the study (3.5 vs 2.9, $p=0.033$; not tabulated). Education, income, and living situation of dropouts and completers were comparable. After the 12-month follow-up period, the intervention and 


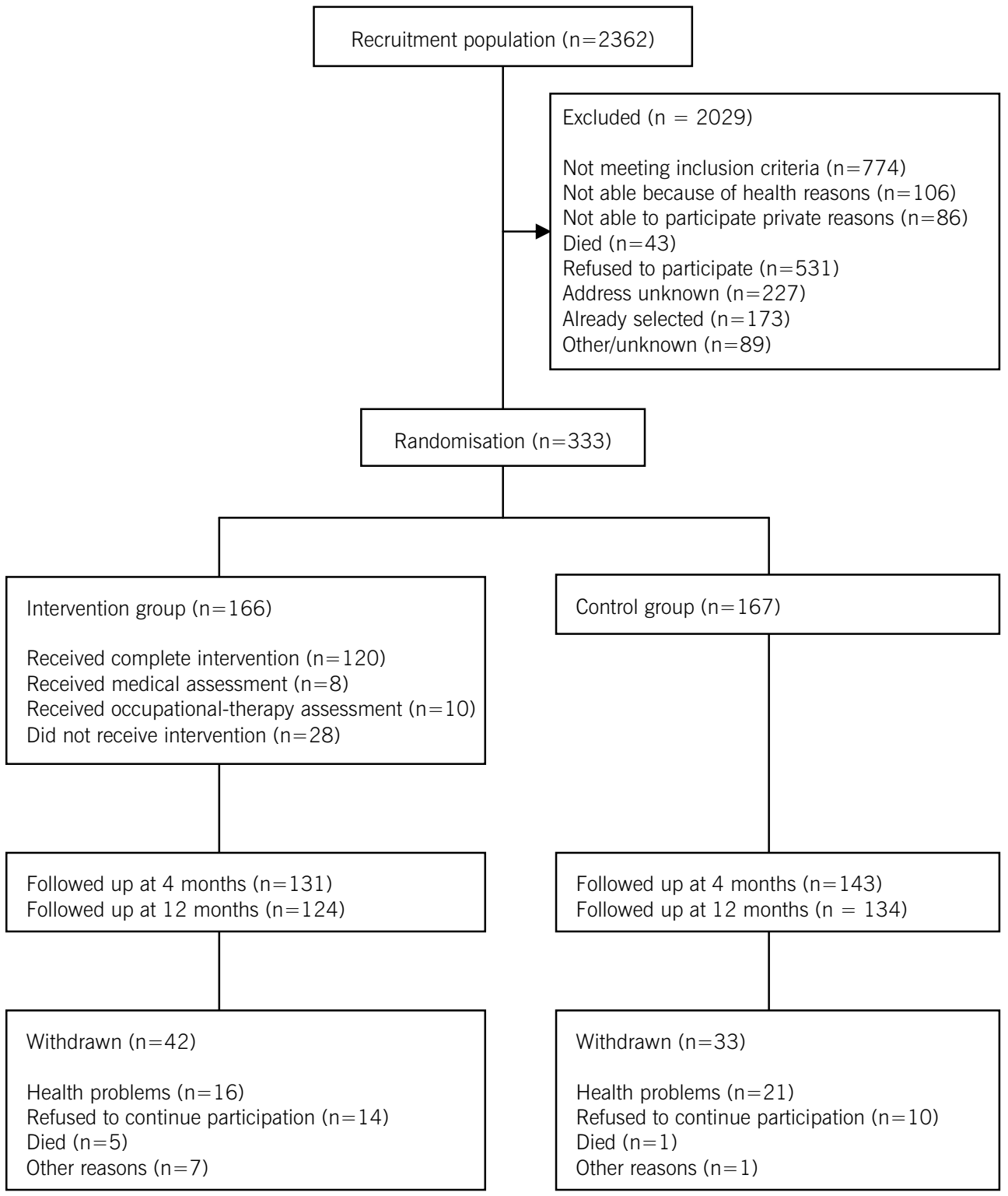

Figure 1 Flow of participants 
control groups remained comparable in terms of background characteristics, except for level of education (data not tabulated). Dropouts from the intervention group had a higher percentage of less-educated participants than completers (38\% vs 21\%). After 4 and 12 months, data on 274 (80\%) and 258 (77\%) participants, respectively, were available for intention-to-treat analysis.

Table 2 - Background characteristics and outcome measures at baseline for the intervention and control groups*

\begin{tabular}{|c|c|c|c|c|}
\hline & \multicolumn{2}{|c|}{$\begin{array}{l}\text { Intervention group } \\
(\mathrm{n}=166)\end{array}$} & \multicolumn{2}{|c|}{$\begin{array}{l}\text { Usual care group } \\
(\mathrm{n}=167)\end{array}$} \\
\hline \multicolumn{5}{|l|}{ Background characteristics } \\
\hline Mean (sd) age & 74.5 & $(5.9)$ & 75.2 & $(6.9)$ \\
\hline Female & 111 & $(66.9)$ & 117 & $(70.1)$ \\
\hline Living alone & 73 & $(44.2)$ & 71 & $(42.5)$ \\
\hline$\leq$ primary school education & 42 & $(25.3)$ & 52 & $(31.1)$ \\
\hline Major injury from index fall (fracture or joint dislocation) & 66 & $(39.8)$ & 60 & $(35.9)$ \\
\hline Mean (sd) self-reported weight & 73.4 & $(13.7)$ & 71.8 & $(12.3)$ \\
\hline $\begin{array}{l}\text { Mean (sd) psychological consequences of the fall } \\
\text { (Falls Handicap Inventory) }\end{array}$ & 22.4 & $(20.7)$ & 22.8 & $(19.6)$ \\
\hline Mean (sd) number of illnesses & 2.8 & $(2.0)$ & 3.2 & $(2.5)$ \\
\hline \multicolumn{5}{|l|}{ Outcome measures } \\
\hline $\begin{array}{l}\text { Persons who had sustained at least one fall in the } \\
\text { previous year }\end{array}$ & 166 & $(100)$ & 167 & $(100)$ \\
\hline $\begin{array}{l}\text { Persons who had sustained more than one fall in the } \\
\text { previous year }\end{array}$ & 82 & $(49.4)$ & 82 & $(49.1)$ \\
\hline Mean (sd) daily functioning (Frenchay Activity Index) & 23.2 & $(8.7)$ & 23.7 & $(8.6)$ \\
\hline
\end{tabular}

*values are number (percentages) unless stated otherwise 


\section{Outcomes}

One hundred and thirty-eight (83\%) of the participants in the intervention group received at least one of the assessments: 120 (72\%) received the medical and OT assessments, 10 only the medical assessment, and eight only the occupational. Ninety-seven percent of the items of both assessments were conducted according to protocol. The medical assessment and the home visit took place within an average of 5 and 10 weeks after baseline, respectively. The results and recommendations were sent to the GPs an average of 3.5 months after baseline. A total of 50 (intended) referrals and 25 recommendations resulted from the medical assessment and were included in the geriatrician's letter to the GP. Of those receiving the medical assessments $(n=130), 56(43 \%)$ received at least one recommendation or referral. Of those receiving the OT assessments $(n=128), 117(91 \%)$ received 456 recommendations. Overall the assessments resulted in at least one referral or recommendation for $89 \%$ ( $n=123$ ) of the participants who received at least one of the assessments $(n=138)$. The process evaluation (33) revealed that only half of the participants asked their GP about the referrals and recommendations, and one-quarter of them did not receive the intended referrals and recommendations from their GP. When the referrals and recommendations reached the participants, self-reported compliance with referrals and recommendations from the medical assessments was $75 \%$. The self-reported compliance with the recommendations the participants reported to have received from the OT assessment was also 75\%.

No significant differences between the two groups were observed in terms of falls or daily functioning (tables 3 and 4 ) after 4 and 12 months. The analysis of falls and recurrent falls for the time after the programme was implemented (5-12 months), and the complete case analysis of falls and recurrent falls showed no significant differences between the groups either (data not tabulated).

Survival analyses showed no differences in time to first fall between the groups for the total follow-up period (1-12 months, $\operatorname{Exp}(B)=1.08 ; p=0.66)$, or for the time after the programme was implemented (5-12 months, $\operatorname{Exp}(B)=0.79 ; p=0.30$, not tabulated).

As regards the secondary outcomes, we found no differences between the groups at 4 or 12 months (tables 3 and 4). No adverse events or side effects were reported. Subsequently, per-protocol comparisons were performed between all participants in the 


\section{Table 3 - Effects on dichotomous outcomes after 4 and 12 months of follow-up}

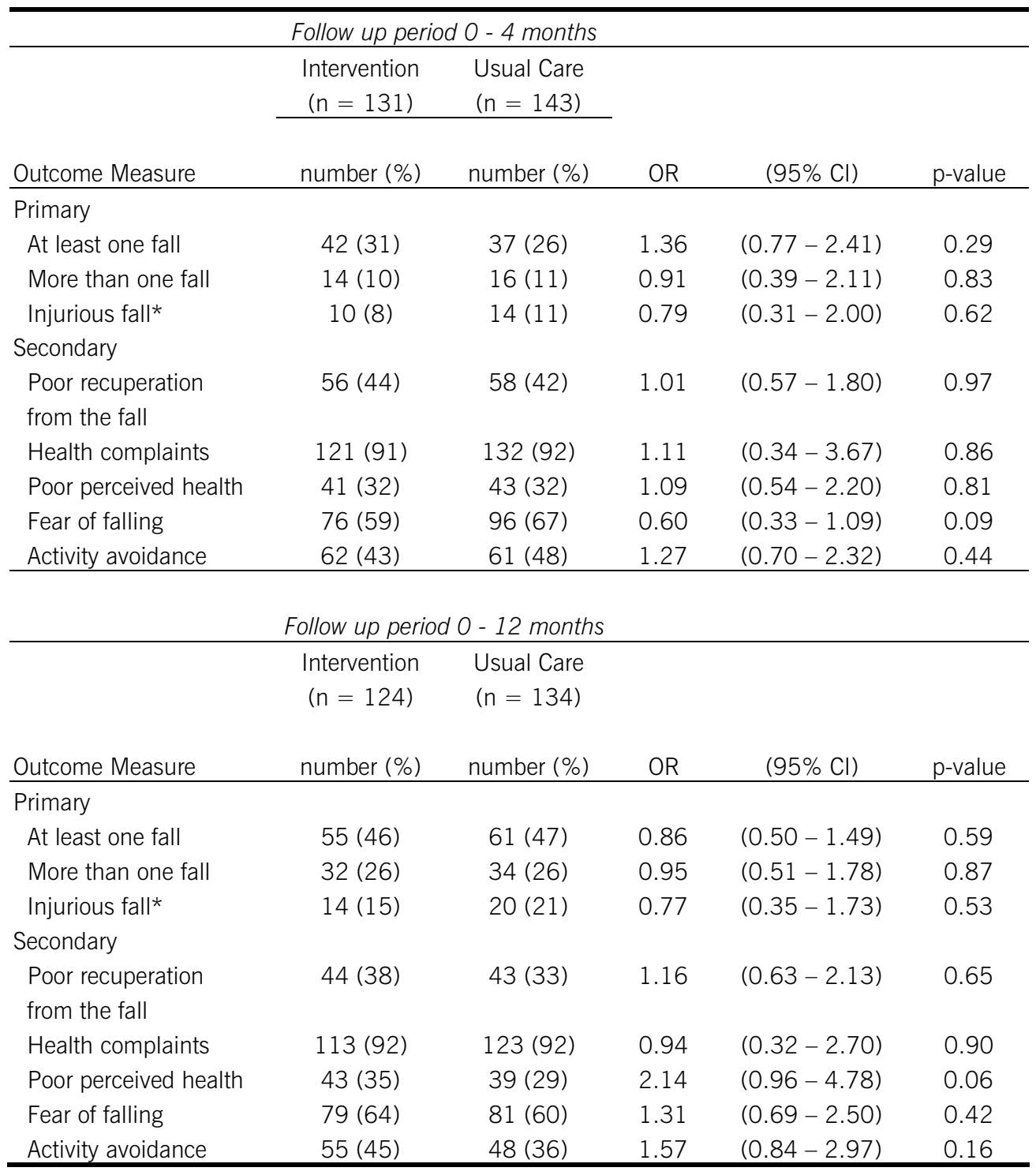

Effects were assessed using multiple logistic regression analysis. Patients who completed at least $75 \%$ of the fall calendar were included in the analysis. Missing calendar months were replaced by individual mean of valid calendar months. * Complete case analysis was performed.

$\mathrm{OR}=$ odds ratio, $\mathrm{Cl}=$ confidence interval 
Table 4 - Effects on continuous outcomes after 4 and 12 months of follow-up

\begin{tabular}{|c|c|c|c|c|c|}
\hline \multirow[b]{3}{*}{ Outcome Measure } & \multicolumn{2}{|c|}{ Follow up period 0 - 4 months } & \multirow[b]{3}{*}{$\mathrm{B}$} & \multirow[b]{3}{*}{$(95 \% \mathrm{Cl})$} & \multirow[b]{3}{*}{$\mathrm{p}$-value } \\
\hline & $\begin{array}{l}\text { Intervention } \\
(\mathrm{n}=131)\end{array}$ & $\begin{array}{l}\text { Usual Care } \\
(\mathrm{n}=143)\end{array}$ & & & \\
\hline & Mean \pm SD & Mean \pm SD & & & \\
\hline \multicolumn{6}{|l|}{ Primary } \\
\hline $\begin{array}{l}\text { Daily functioning (FAI) } \\
(0-45)^{*}\end{array}$ & $25.2 \pm 7.3$ & $24.6 \pm 8.1$ & 0.40 & $(-0.74-1.53)$ & 0.49 \\
\hline \multicolumn{6}{|l|}{ Secondary } \\
\hline Mental Health $(0-42)^{\dagger}$ & $10.2 \pm 7.4$ & $9.9 \pm 7.1$ & 0.59 & $(-0.40-1.57)$ & 0.24 \\
\hline $\begin{array}{l}\text { Activity of daily living } \\
\text { and instrumental } \\
\text { activity of daily living } \\
\text { disability }(11-44)^{\dagger}\end{array}$ & $15.7 \pm 5.5$ & $15.5 \pm 5.5$ & 0.46 & $(-0.22-1.47)$ & 0.18 \\
\hline $\begin{array}{l}\text { Social participation } \\
(2-12)^{\dagger}\end{array}$ & $6.5 \pm 2.3$ & $6.3 \pm 1.9$ & 0.05 & $(-0.36-0.46)$ & 0.80 \\
\hline EuroQol & $0.71 \pm 0.25$ & $0.72 \pm 0.27$ & -0.03 & $(-0.07-0.01)$ & 0.13 \\
\hline \multicolumn{6}{|c|}{ Follow up period $0-12$ months } \\
\hline & $\begin{array}{c}\text { Intervention } \\
(\mathrm{n}=124)\end{array}$ & $\begin{array}{l}\text { Usual Care } \\
(n=134)\end{array}$ & & & \\
\hline Outcome Measure & Mean $\pm \mathrm{SD}$ & Mean $\pm \mathrm{SD}$ & $\mathrm{B}$ & $(95 \% \mathrm{Cl})$ & $\mathrm{p}$-value \\
\hline \multicolumn{6}{|l|}{ Primary } \\
\hline $\begin{array}{l}\text { Daily functioning (FAI) } \\
(0-45)^{*}\end{array}$ & $25.6 \pm 8.0$ & $24.5 \pm 9.1$ & 0.37 & $(-0.90-1.63)$ & 0.57 \\
\hline \multicolumn{6}{|l|}{ Secondary } \\
\hline Mental Health $(0-42)^{\dagger}$ & $10.4 \pm 8.3$ & $10.0 \pm 7.6$ & 0.59 & $(-0.65-1.83)$ & 0.35 \\
\hline $\begin{array}{l}\text { Activity of daily living } \\
\text { and instrumental } \\
\text { activity of daily living } \\
\text { disability }(11-44)^{\dagger}\end{array}$ & $15.2 \pm 1.8$ & $15.4 \pm 5.6$ & -0.03 & $(-0.64-0.64)$ & 0.94 \\
\hline $\begin{array}{l}\text { Social participation } \\
(2-12)^{\dagger}\end{array}$ & $6.3 \pm 2.4$ & $6.1 \pm 2.0$ & -0.07 & $(-0.52-0.37)$ & 0.75 \\
\hline EuroQol & $0.70 \pm 0.25$ & $0.71 \pm 0.28$ & -0.012 & $(-0.06-0.03)$ & 0.59 \\
\hline
\end{tabular}

Outcomes were assessed using multiple linear regression analysis.

* Higher score is the more favourable score. $\dagger$ Lower score is the more favourable score.

$\mathrm{OR}=$ odds ratio, $\mathrm{Cl}=$ confidence interval 
intervention group who had received both the medical and OT assessments ( $n=120 ; 72 \%$ ) and the complete control group. These analyses showed no significant differences on any outcome measure (data not shown). Furthermore, subgroup analyses did not show any differences in terms of falls between the subgroups in the intervention and control groups (data not shown). Only daily functioning (FAI) significantly improved in participants aged 80 years and over allocated to the intervention group (control $n=29$ vs intervention $n=22$; $B$ $=4.134 ; \mathrm{p}=0.019$; not tabulated).

\section{CONCLUSION AND DISCUSSION}

No effect was found of the programme on falls, daily functioning or secondary outcome measures in the intention to treat analyses. The analyses in which the subjects who received the complete intervention were compared with the control group (per-protocol analyses) did not show any effects in falls or daily functioning either. Separate analyses of falls for the time after the programme was implemented (5-12 months), and complete case analyses (including those participants with complete data) did not show any differences either. These findings are in sharp contrast with those from a previous study (2) in the United Kingdom, which found that the multidisciplinary fall prevention programme on which this programme was bases had favourable effects on the number of fallers and daily functioning. Furthermore, the results do not corroborate the conclusion of another study that multifactorial risk assessment and management programmes generally appear to be effective in reducing falls $(1,4,5)$, although that study found that the effects are generally small and mentioned the problem of heterogeneity of results, indicating that differences in the status of the participants, the context, and details of the content and presentation of the intervention can probably explain this heterogeneity (1). Moreover, a recent systematic review on multifactorial assessment and prevention programmes concluded that the evidence of benefit from these assessments and prevention programmes may be smaller than thought (34).

There may be several explanations for the ineffectiveness of this programme and the marked difference between our results and those of the British trial. First, the essential adaptations to the protocol, in order to integrate the programme as much as possible into routine Dutch health care may have influenced the results of this trial. Two disciplines (rehabilitation physician 
and geriatric nurse) were added to the medical assessment team, and GPs were involved in the programme. This resulted in an extended implementation period of approximately 3.5 months. This relatively long time between the fall and completion of the programme may have reduced the effectiveness of the programme, but shortening this period would be a difficult without deviating from routine procedures in the Netherlands.

Second, some of the GPs involved in the trial had patients in both the intervention and the control groups in their practices. Therefore, it is possible that the programme influenced the way these GPs managed falls in their practices, although because the referrals and the recommendations resulted from an individualised assessment, it is likely that this possible contamination effect was negligible. Moreover, the GPs were not aware of which patients participated in the control group.

Third, differences in the trial populations can explain the differences between the outcomes of the two studies. It is possible that patients enrolled in the British trial were more at risk of falls or more underserved and therefore more likely to benefit from the programme than the patients assessed in the current study. Although the same selection procedure was performed, the current study population was on average somewhat younger than the population selected for the British study (74.9 vs 78.2 years) (2). Moreover, more people died in the British study than in the current study ( $n=46,12 \%$ vs $n=6,2 \%$ ) (2), indicating that the British sample consisted of frailer persons. However, the number of recurrent fallers in the control group in the current study was comparable to the findings of the control group of the British and other studies $(2,35,36)$. Subgroup analyses of participants who were older and more at risk did not reveal favourable effects of the programme on falls either. With regard to the available healthcare services, it has been said that London's health services do not sufficiently meet the needs of Londoners (37). This implies that the programme may have added more to routine care in London than to regular care in a Dutch setting, although in the current study, for $11 \%$ of the participants, no further action was recommended, whereas in the British study, $16 \%$ of the participants required no further action. In that study, in only 24 (16\%) of the assessments no further action was required (2). Unfortunately, further comparison of the contents of the referral and recommendations resulting from the two trials is difficult, because the British study does not report on the details of the referrals or recommendations. 
Fourth, deviations from the protocol may have influenced the effectiveness of the programme, although a detailed process evaluation performed alongside the trial revealed that the programme was largely performed according to protocol (with 97\% of the protocol items performed as planned), and those who administered the assessments considered it to be feasible. This makes it highly unlikely that deviations from protocol can explain the absence of favourable effects.

Fifth, a lack of compliance with the programme may have influenced the outcomes. Only half of the participants who underwent the medical assessment called their GP, and one-quarter of them did not receive the intended referrals and recommendations from their GP. Therefore many participants did not receive intended interventions. Recommending to participants that they contact their GP, and subsequently sending them a reminder was apparently not an effective way to make them call their GP. Lack of time or differences of opinion, but also by overlap between the suggestions made by the geriatrician and the care and services already provided to these patients, may have led the GPs not to follow the geriatrician's suggestions in one- quarter of the cases (38-41). The self-reported compliance with the referrals and recommendations that reached the participants was $75 \%$, which seems reasonably good. Because the British study (2) did not report on the compliance rate in their programme, it is not possible to compare the two studies in this respect.

Overall, a multidisciplinary fall prevention programme in community-dwelling older people was not effective in the Dutch healthcare setting. Therefore, implementation of this programme in its present form is not recommended in usual care in the Netherlands. Involving the GP and extending the implementation period was in accordance with routine care in the Netherlands, but it may have reduced the effectiveness of the programme. There are some indications that higher intensity interventions that provide direct action (e.g., treatments) to address fall risk factors may be more effective than interventions that provide information and referral (34). Therefore, searching for possibilities to overcome the problem of nonacute referral is recommended to increase the efficiency and effectiveness of the programme. Furthermore, mechanisms need to be found to enhance compliance of participants with the recommendations. The current study revealed that proven effectiveness of a prevention programme in one healthcare setting is no guarantee for its effectiveness in another setting. 
Moreover, there can be considerable discrepancy between an experimental version of a programme and the implemented version of the same programme. The importance of implementation research assessing feasibility and effectiveness of a programme in specific healthcare settings is stressed. To achieve effective fall prevention in practice, the barriers to implementation need to be studied and these problems overcome (41).

\section{ACKNOWLEDGEMENTS AND FUNDING}

We would like to thank the following persons for their contributions: Charlotte H. MomSchuurmann, CIZ Centre healthcare indications in the Netherlands; MEMIC, Centre for Data and Information Management; Maastricht University Hospital; Municipal authorities of Maastricht. This study was funded by the Netherlands Organization for Health Research and Development (ZonMw), grant number 945-02-053. P.O. Box 93 245, 2509 AE Den Haag, The Netherlands. The funding source had no involvement in the data collection or analyses 


\section{REFERENCES}

1. Gillespie $D$, Gillespie WJ, Robertson MC, Lamb SE, Cumming RG, Rowe BH. Interventions for preventing falls in elderly people. Cochrane Database Syst Rev. 2003(4):CD000340.

2. Close J, Ellis M, Hooper R, Glucksman E, Jackson S, Swift C. Prevention of falls in the elderly trial (PROFET): a randomised controlled trial. Lancet. 1999 Jan 9;353(9147):93-7.

3. Rizzo JA, Friedkin R, Williams CS, Nabors J, Acampora D, Tinetti ME. Health care utilization and costs in a Medicare population by fall status. Med Care. 1998 Aug;36(8):1174-88.

4. Chang JT, Morton SC, Rubenstein LZ, Mojica WA, Maglione M, Suttorp MJ, et al. Interventions for the prevention of falls in older adults: systematic review and meta-analysis of randomised clinical trials. Bmj. 2004;328(7441):680.

5. Kannus P, Sievanen H, Palvanen M, Jarvinen T, Parkkari J. Prevention of falls and consequent injuries in elderly people. Lancet. 2005 Nov 26;366(9500):1885-93.

6. Kellogg International Work Group on the Prevention of Falls by the Elderly. The prevention of falls in later life. Dan Med Bull. 1987;34 Suppl 4:1-24.

7. Hendriks MR, van Haastregt JC, Diederiks JP, Evers SM, Crebolder HF, van Eijk JT. Effectiveness and costeffectiveness of a multidisciplinary intervention program to prevent new falls and functional decline among elderly persons at risk: design of a replicated randomised controlled trial [ISRCTN64716113]. BMC Public Health. 2005 Jan 14;5:6.

8. van Uden CJ, Winkens RA, Wesseling GJ, Crebolder HF, van Schayck CP. Use of out of hours services: a comparison between two organisations. Emerg Med J. 2003;20(2):184-7.

9. Swain DG, Nightingale PG. Evaluation of a shortened version of the Abbreviated Mental Test in a series of elderly patients. Clin Rehabil. 1997;11(3):243-8.

10. Hendriks MR, Bleijlevens MH, van Haastregt JC, de Bruijn FH, Diederiks JP, Mulder WJ, et al. A multidisciplinary fall prevention program for elderly persons: a feasibility study. Geriatr Nurs. 2008 MayJun;29(3):186-96.

11. Hetherington R. The Snellen chart as a test of visual acuity. Psychol Forsch. 1954;24(4):349-57.

12. Walraven J. Amblyopia screening with random-dot stereograms. Am J Ophthalmol. 1975 Nov;80(5):893-900.

13. van der Ploeg RJ, Oosterhuis HJ. Fysische diagnostiek--het meten van spierkracht. [Physical examination-measurement of muscle strength]. Ned Tijdschr Geneeskd. 2001 Jan 6;145(1):19-23.

14. Menz HB, Sherrington C. The Footwear Assessment Form: a reliable clinical tool to assess footwear characteristics of relevance to postural stability in older adults. Clin Rehabil. 2000;14(6):657-64. 
15. Van Vaerenbergh J, Broos P. Positieve Romberg-test en het voorspellen van vallen bij bejaarden. [Positive Romberg test and the probability of falls in the aged]. Tijdschr Gerontol Geriatr. 1990 Apr;21(2):71-4.

16. Mathias S, Nayak US, Isaacs B. Balance in elderly patients: the "get-up and go" test. Arch Phys Med Rehabil. 1986;67(6):387-9.

17. Folstein MF, Folstein SE, McHugh PR. "Mini-mental state". A practical method for grading the cognitive state of patients for the clinician. J Psychiatr Res. 1975 Nov; 12(3):189-98.

18. Brink TL, Yesavage JA, Lum O, Heersema PH, Adey M, Rose TL. Screening tests for geriatric depression. Clin gerontologist. 1982;1(1):37-43.

19. Kulu Glasgow I, Delnoij D, de Bakker D. Self-referral in a gatekeeping system: patients' reasons for skipping the general-practitioner. Health policy. 1998 Sep;45(3):221-38.

20. Schuling J, de Haan R, Limburg M, Groenier KH. The Frenchay Activities Index. Assessment of functional status in stroke patients. Stroke. 1993 Aug;24(8):1173-7.

21. Rooken M, Van der Ven S. Standaard voor het afleggen van een huisbezoek door ergotherapeuten: Stroomschema en formulier. Utrecht: Nederlandse Vereniging voor Ergotherapie; 2001.

22. Ummels C, Lahaye V. Ergotherapeutische registratielijst voor een ADL-zelf-verzorgingsobservatie: Handleiding en formulier. Utrecht: Nederlandse Vereniging voor Ergotherapie; 2001.

23. Stalenhoef P, Diederiks J, Knottnerus A, de Witte L, Crebolder H. How predictive is a home-safety checklist for indoor fall risk in the elderly living in the community? Eur J Gen Pract. 1998;4:114-20.

24. Rai GS, Kiniorns M, Wientjes H. Falls Handicap Inventory (FHI)--an instrument to measure handicaps associated with repeated falls. J Am Geriatr Soc. 1995;43(6):723-4.

25. Russell MA, Hill KD, Blackberry I, Day LL, Dharmage SC. Falls risk and functional decline in older fallers discharged directly from emergency departments. J Gerontol A Biol Sci Med Sci. 2006 Oct;61(10):1090-5.

26. van der Zee KI, Sanderman R. Het meten van de algemene gezondheidstoestand met de RAND-36: een handleiding [Measuring general health with the RAND-36:manual]. Groningen: Noordelijk Centrum voor Gezondheidsvraagstukken, Rijksuniversiteit Groningen; 1993.

27. Kempen GI, Miedema I, Ormel J, Molenaar W. The assessment of disability with the Groningen Activity Restriction Scale. Conceptual framework and psychometric properties. Soc Sci Med. 1996 Dec;43(11):1601-10.

28. Spinhoven P, Ormel J, Sloekers PP, Kempen GI, Speckens AE, Van Hemert AM. A validation study of the Hospital Anxiety and Depression Scale (HADS) in different groups of Dutch subjects. Psychol Med. 1997 Mar;27(2):363-70.

29. Brooks R. Euroqol:the current state of play. Health policy. $1996 \mathrm{Jul} ; 37(1): 53-72$.

30. Dolan P. Modeling valuations for EuroQol health states. Med Care. 1997;35(11):1095-108. 
31. Tinetti ME, Speechley M, Ginter SF. Risk factors for falls among elderly persons living in the community. N Engl J Med. 1988;319(26):1701-7.

32. Campbell AJ, Borrie MJ, Spears GF. Risk factors for falls in a community-based prospective study of people 70 years and older. J Gerontol. 1989;44(4):M112-7.

33. Bleijlevens MH, Hendriks MR, van Haastregt JC, van Rossum E, Kempen GI, Diederiks JP, et al. Process factors explaining the ineffectiveness of a multidisciplinary fall prevention programme: a process evaluation. BMC Public Health. 2008 Sep 24;8(1):332.

34. Gates S, Lamb SE, Fisher JD, Cooke MW, Carter YH. Multifactorial assessment and targeted intervention for preventing falls and injuries among older people in community and emergency care settings: systematic review and meta-analysis. Bmj. 2008 Jan 19;336(7636):130-3.

35. Tinetti ME, Baker DI, McAvay G, Claus EB, Garrett P, Gottschalk M, et al. A multifactorial intervention to reduce the risk of falling among elderly people living in the community. N Eng/ J Med. 1994;331(13):821-7.

36. O'Loughlin J, Robitaille Y, Bolvin J-F, Suissa S. Incidence of and risk factors for falls and injurious falls among the community-dwelling elderly. Am J Epidemiol. 1993;137:342-54.

37. Thorlby R, Dixon J, Dickson N. Health for London: showing England the way? Bmj. 2007 Jul 21;335(7611):108-9.

38. Yarnall KS, Pollak KI, Ostbye T, Krause KM, Michener JL. Primary care: is there enough time for prevention? Am J Public Health. 2003 Apr;93(4):635-41.

39. Fortinsky RH, lannuzzi-Sucich M, Baker DI, Gottschalk M, King MB, Brown CJ, et al. Fall-risk assessment and management in clinical practice: views from healthcare providers. J Am Geriatr Soc. 2004 Sep;52(9):1522-6.

40. Chou WC, Tinetti ME, King MB, Irwin K, Fortinsky RH. Perceptions of physicians on the barriers and facilitators to integrating fall risk evaluation and management into practice. J Gen Intern Med. 2006 Feb;21(2):117-22.

41. Tinetti ME, Gordon C, Sogolow E, Lapin P, Bradley EH. Fall-risk evaluation and management: challenges in adopting geriatric care practices. Gerontologist. 2006 Dec;46(6):717-25. 



\section{PROCESS FACTORS EXPLAINING THE}

INEFFECTIVENESS OF A MULTIDISCIPLINARY FALL

PREVENTION PROGRAMME:

A PROCESS EVALUATION

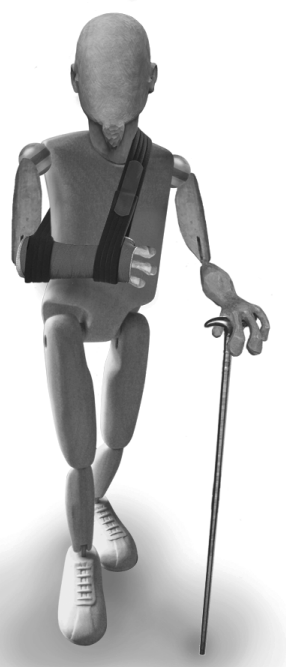

Michel HC Bleijlevens

Marike RC Hendriks

Jolanda CM van Haastregt

Erik van Rossum

Gertrudis IJM Kempen

Joseph PM Diederiks

Harry FJM Crebolder

Jacques ThM van Eijk

BMC Public Health 2008; 8(1):332 


\section{ABSTRACT}

\section{Background}

Falls are a major health threat to older community-dwelling people, and initiatives to prevent falls should be a public health priority. We evaluated a Dutch version of a successful British fall prevention programme. Results of this Dutch study showed no effects on falls or daily functioning. In parallel to the effect evaluation, we carried out a detailed process evaluation to assess the feasibility of our multidisciplinary fall prevention programme. The present study reports on the results of this process evaluation.

\section{Methods}

Our fall prevention programme comprised a medical and occupational-therapy assessment, resulting in recommendations and/or referrals to other services if indicated. We used self-administered questionnaires, structured telephone interviews, structured recording forms, structured face-to-face interviews and a plenary group discussion to collect data from participants allocated to the intervention group $(n=166)$ and from all practitioners who performed the assessments $(n=8)$. The following outcomes were assessed: the extent to which the multidisciplinary fall prevention programme was performed according to protocol, the nature of the recommendations and referrals provided to the participants, participants' self-reported compliance and participants' and practitioners' opinions about the programme.

\section{Results}

Both participants and practitioners judged the programme to be feasible. The programme was largely performed according to protocol. The number of referrals and recommendations ensuing from the medical assessment was relatively small. Participants' self-reported compliance as regards contacting their GP to be informed of the recommendations and/or referrals was low to moderate. However, self-reported compliance with such referrals and recommendations was reasonable to good. A large majority of participants reported they had benefited from the programme.

\section{Conclusion}

The results of the present study show that the programme was feasible for both practitioners and participants. Main factors that seem to be responsible for the lack of effectiveness are the relatively low number of referrals and recommendations ensuing from the medical assessments and participants' low compliance as regards contacting their GP about the results of the medical assessment. We do not recommend implementing the programme in its present form in regular care. 


\section{BACKGROUND}

Falls are a major health threat to older people living in the community, and initiatives to prevent these falls should be a public health priority. Approximately one third of community-dwelling people aged 65 and over fall at least once a year (1-6). About one fifth of all falls result in an injury that requires medical attention, and about one tenth lead to serious physical consequences, such as fractures, joint dislocations and lacerations (6-9). In addition, falls can have considerable psychosocial consequences, like fear of falling, depression and social isolation (10-12). Together, these physical and psychosocial consequences are responsible for reduced physical activity (11, $13)$, early admission to hospital or nursing home $(2,14)$, increased mortality and morbidity $(14,15)$ and loss of autonomy $(2,10)$.

Close and colleagues developed a multidisciplinary fall prevention programme aimed at community-dwelling people aged 65 years and over who had visited the accident and emergency (A\&E) department because of a fall $(16,17)$. Although this programme showed promising effects in this British setting, this is no guarantee for its effectiveness in other healthcare settings. We therefore developed a Dutch version of this successful programme and tested its effect on falls and daily functioning by means of a randomised controlled trial (17). The results of this trial showed that the programme did not have any effect on falls or daily functioning (18). In parallel to this randomised controlled trial, we carried out a detailed process evaluation primarily aimed at assessing the feasibility of our multidisciplinary programme. The second aim of this process evaluation was to identify factors which might explain the lack of effectiveness of our programme. This paper presents the results of this process evaluation. We translated the two aims of our evaluation into the following four specific research questions:

1. To what extent was the fall prevention programme performed according to protocol?

2. What was the nature of the recommendations and referrals made to the participants?

3. What was the participants' self-reported compliance?

4. What are the participants' and practitioners' opinions about the programme? 


\section{METHODS}

\section{Fall prevention programme}

The fall prevention programme consisted of a medical and occupational-therapy assessment, followed by recommendations or further referral if indicated. The medical assessment consisted of examinations performed by a geriatrician, a geriatric nurse and a rehabilitation physician at the hospital (17). The assessment included a comprehensive general examination and a detailed assessment of vision, sense of hearing, locomotor apparatus, feet and footwear, peripheral nervous system, mobility, balance, anthropometry, cognition, affect, blood test if indicated and medication use. On completion of the medical assessment, the geriatrician evaluated the results and sent a written summary to the participant's general practitioner (GP). This letter included recommendations and/or referrals to relevant services, if necessary. The participants were advised to contact their GP to be informed of the results of the medical assessment and the recommendations and/or referrals to other services ensuing from it.

The occupational-therapy assessment was performed by an occupational therapist at the participant's home and comprised a functional and environmental assessment (17). On completion of this assessment, recommendations with regard to behavioural change, functional needs and safety within the home environment were immediately given to the patient. Recommendations and referrals concerning technical aids and adaptations or additional support to be provided by social and community services were implemented in accordance with the procedures prevailing in regular care. The participants received a letter with the recommendations and/or referrals, by way of reminder. A copy was sent to the participants' GPs, to inform them of the results of the assessment.

\section{Usual care}

The participants who were allocated to the control group of the randomised controlled trial and for that reason did not underwent the fall prevention programme, received usual care. During the trial, no standard approach to fall risk assessment was available for fallers presenting to the A\&E department and being discharged home. In usual care in the Netherlands, medical risks and other risk factors for falls, such as environmental hazards in the home and patients' risk behaviour, are not systematically registered and addressed by hospital physicians, medical specialists or general practitioners. Moreover, when people present to the A\&E department with the consequences of 
an injurious fall, in general no systematic attention is being paid to the specific consequences of that fall for daily functioning of individual patients in their unique situation.

\section{Study population}

The study population of this process evaluation can be divided into two groups:

1. All 166 participants allocated to the intervention group (referred to below as participants).

2. The medical and paramedical practitioners who performed the medical and occupationaltherapy assessments (one geriatrician, three geriatric nurses, two rehabilitation physicians and two occupational therapists) (referred to below as practitioners) (17).

\section{Data collection}

Table 1 shows the aspects of the intervention process that were assessed and the methods used. Data were collected from participants by means of self-administered questionnaires and structured interviews by telephone. Independent assistants asked the participants to fill out a questionnaire immediately after the medical assessment in order to assess their opinion about this assessment. For practical reasons and to avoid social desirable answers, the participants did not receive a questionnaire from the occupational therapist immediately after the occupational-therapy assessment. In order to assess the participants' opinion about the occupational-therapy assessment, detailed questions about this assessment were embedded in the structured telephone interviews which took place about six months after the recommendations and referrals had been sent to the GPs. These telephone interviews also comprised questions assessing participants' compliance with the referrals and recommendations and their overall opinion about the programme.

We used structured recording forms, structured face-to-face interviews and a plenary group discussion to collect data from the practitioners regarding the performance according to protocol, the nature of the recommendations and referrals, the compliance of the participants with the referrals and recommendations, and their opinion about the programme. The recording forms were filled out by the practitioners during or immediately after the assessments. The structured face-to-face interviews with the practitioners were scheduled immediately after all participants had undergone the assessments, and the plenary group discussion with the practitioners and the research team was carried out six months after all participants had undergone the assessments. 


\section{Table 1 - Outcome measures and measurement instruments of the process evaluation}

Events in chronological order $\rightarrow$

$\begin{array}{lllllll}R & Q & \mathrm{FI} & \mathrm{L} & \mathrm{T} & \mathrm{PD}\end{array}$

Performance of programme according to protocol

Deviations from protocol

Timing and duration of the assessments

Nature of recommendations and referrals from assessments

Participants' compliance with referrals and recommendations

Self-reported compliance with contacting GP

Self-reported compliance with referrals and

recommendations resulting from the medical assessment

Self-reported compliance with recommendations resulting

from the occupational-therapy assessment

Opinion about the programme

Benefit and satisfaction experienced by the participants

Practicability of the recording forms

Acceptability of the programme to participants

Recommendations (for implementation)

$\begin{array}{llll}x & & x & x \\ x & x & x & x\end{array}$

$x$

$X$

$X$

X $\quad x$

$x$

$X$

$x \quad x$

$\mathrm{R}=$ Structured recording forms for the practitioners regarding the medical and occupational-therapy assessments;

$Q=$ Self-administered questionnaires for all participants who underwent the medical assessment; $\mathrm{Fl}=$ Structured individual face-to-face interviews with the practitioners; $L=$ Letters written by the geriatrician and occupational therapists to GPs, listing recommendations and/or referrals; $T$ = Structured interviews by telephone with the participants who underwent the medical and/or the occupational-therapy assessment, about 6 months after the recommendations ensuing from the assessment(s) had been sent to the GP; PD = Plenary group discussion with the practitioners and the research team. 


\section{Data analysis}

Quantitative data (e.g. duration of the assessments, perceived benefit) were analysed by means of descriptive statistics. Qualitative data (i.e. answers to open questions in the selfadministered questionnaires, individual interviews and the plenary group discussion) were classified into categories, based on the content of the answers given.

\section{Ethical considerations}

The Medical Ethics Committee of Maastricht University and the University Hospital Maastricht approved this process evaluation, being a part of the randomised controlled trial (17).

\section{RESULTS}

\section{Attendance and response rate}

The flow of participants through the process evaluation is shown in figure 1. Of the 166 persons allocated to the intervention group, 28 (17\%) did not undergo any assessment because they withdrew from the study before the start of the assessments $(n=27)$ or had a problem with scheduling the assessments $(n=1)$. A total of 138 participants underwent at least one of the two assessments: 120 underwent both assessments, ten only the medical assessment and eight only the occupational-therapy assessment. Reasons for undergoing only one assessment were personal circumstances $(n=14)$ and withdrawal from the study before the occupational-therapy assessment was scheduled $(n=4)$. None of these reasons were related to the programme. All 130 participants who underwent the medical assessment received a self-administered evaluation questionnaire immediately after the medical assessment. The response to this questionnaire was $100 \%$. Of the 138 participants who underwent at least one assessment, thirteen withdrew from the study after completing the medical and/or occupational-therapy assessment. The remaining 125 participants were contacted for a structured interview by phone, about six months after the results of the assessments had been sent to the GPs. Two persons could not be contacted, resulting in a response of $98 \%$. Of these 123 participants, 116 had undergone the medical assessment and 117 had undergone the occupational-therapy assessment.

The practitioners filled in recording forms during the assessments for all 130 participants who underwent the medical assessment and for all 128 participants who underwent the 
Randomisation in randomised controlled trial $(n=333)$
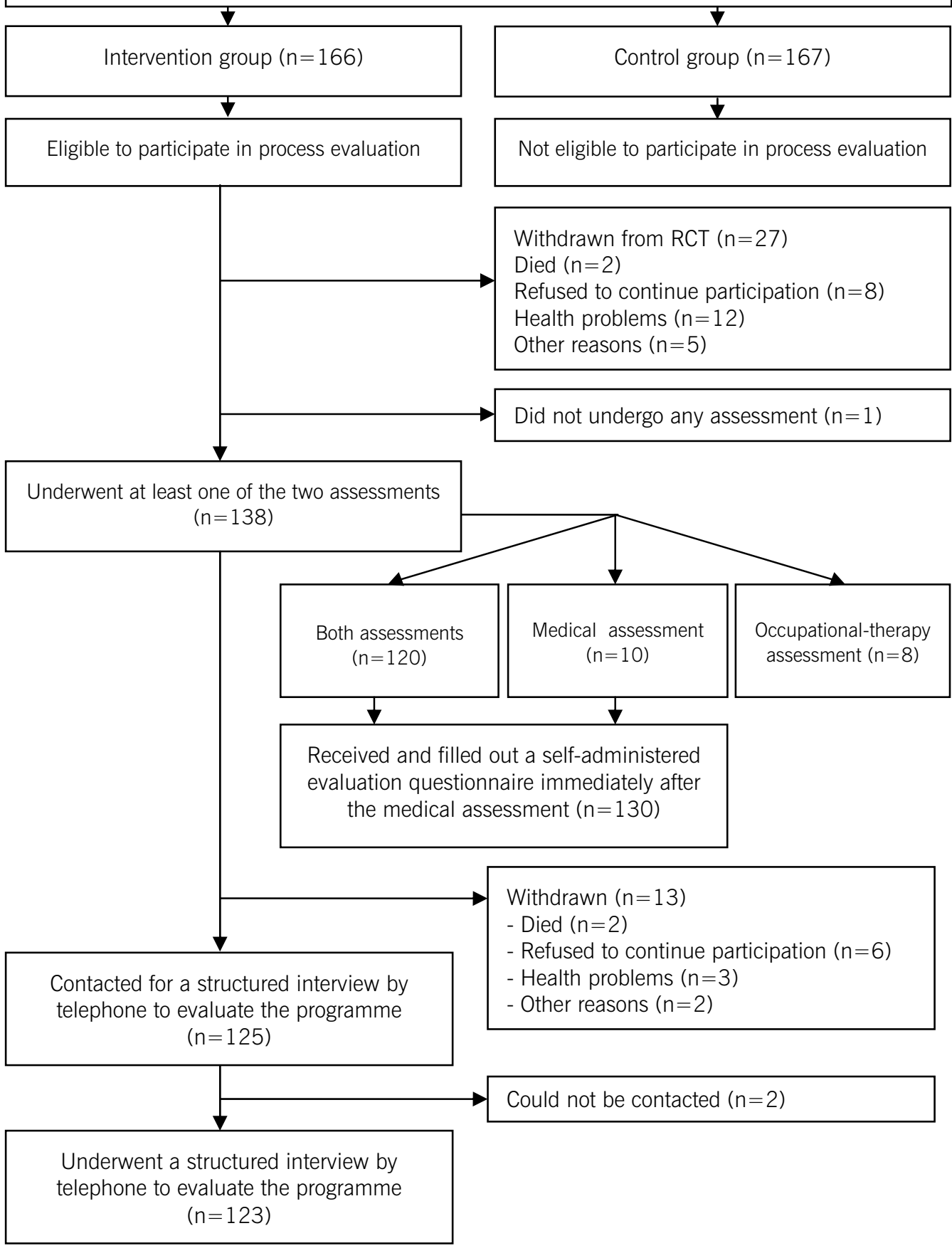

Figure 1 - Flow chart of participants 
occupational-therapy assessment. All but one practitioner (an occupational therapist) took part in the structured face-to-face interviews immediately after the implementation period of the programme. In addition, the practitioners, except one geriatric nurse and one rehabilitation physician, participated in the plenary group discussion six months after the last assessments.

\section{Performance of programme according to protocol}

\section{Protocol deviations}

The recording forms filled in by the practitioners showed that $97 \%$ of the protocol items were carried out according to protocol. Analyzing the recording forms revealed only one minor protocol deviation. During the medical assessment blood pressure was not measured in the erect position (in stead of measuring both sitting and in erect position) in 28 of the 130 participants (22\%). The information obtained from the forms was in agreement with the information gathered during the face-to-face interviews and the plenary group discussion.

\section{Duration of the assessments, time between baseline measurement and sending the letters with recommendations}

The geriatrician, the geriatric nurses and the rehabilitation physicians reported that it took 60 to 90 minutes to perform the medical assessment. The mean amount of time the geriatrician spent processing the referrals and recommendations to the GPs was estimated to be 15 minutes. The mean duration of each occupational-therapy assessment was 55 minutes and the mean time spent on processing a recording form was 21 minutes. The reported time needed for the medical and occupational-therapy assessments was in agreement with the protocol. The period between baseline measurement and sending letters to the GPs with recommendations was on average 3.5 months.

\section{Nature of the recommendations and/or referrals}

\section{Referrals and recommendations resulting from the assessments}

Table 2 shows the nature of the referrals and recommendations ensuing from the medical and occupational-therapy assessments. The referrals and recommendations made by the geriatrician comprised referrals to other specialists or therapists and recommendations concerning measures such as change of medication and orthopaedic footwear. The 
recommendations made by the occupational therapists can be subdivided into four categories: (1) adaptations to the home environment (e.g. installing hand rails, shower chair, raised toilet); (2) behavioural change (e.g. adapting speed of working, using antiskid mats, removing loose rugs, using hand rails); (3) health services (e.g. intake for assistive living, intake for a home for the elderly, GP consultation); and (4) assistive devices (e.g. walking device, lift chair).

As reported by the geriatrician, the medical assessments resulted in 50 referrals and 25 recommendationsfor the 130 participants, which is on average 0.58 referrals/recommendations per participant. Forty-three percent of the participants $(n=56)$ received at least one referral or recommendation, and $57 \%(n=74)$ received no referral or recommendation.

As reported by the occupational therapists, 128 participants received a total of 457 recommendations (3.57 per participant) during the occupational-therapy assessments. For $91 \%$ of the participants ( $n=117$ ), the occupational-therapy assessment resulted in at least one referral or recommendation. For $9 \%(n=11)$, it did not result in any referral or recommendation.

Overall, of the 138 participants who underwent at least one of the two assessments, 123 participants (89\%) received at least one recommendation or referral.

\section{Participants' compliance}

\section{Contact with GP}

Of the 123 persons interviewed by telephone, 7 had not undergone a medical assessment and could therefore not answer the question whether they had contacted their GP. Of the remaining 116 participants, about half $(n=61)$ had contacted their GP to ask for the outcomes of the medical assessment, 45\% $(n=52)$ had not contacted their GP and 3 persons (2\%) did not answer this question. Reasons for not contacting the GP were: forgotten $(n=28)$; not being aware of the possibility to contact the GP $(n=13)$; still intending to contact the GP $(n=6)$; not considering it necessary to contact the GP $(n=4)$ and death $(n=1)$. 
Table 2 - Referrals and recommendations resulting from the medical and occupationaltherapy assessments

Number of R/R resulting

from assessments

Referrals from Medical assessments $(n=130)$

Cardiologist

Osteoporosis examination 8

Orthopaedic shoemaker $\quad 25$

Orthopaedic instrument maker 1

Physiotherapist 4

Other referrals 4

$\begin{array}{ll}\text { Total } & 50\end{array}$

Recommendations from medical assessments $(n=130)$

$\begin{array}{ll}\text { Adjust medication } & 7\end{array}$

Adjust footwear 3

Further examination 8

Vitamin B supplementation 2

Other recommendations 5

$\begin{array}{ll}\text { Total } & 25\end{array}$

Recommendations from occupational-therapy assessments $(n=128)$

$\begin{array}{ll}\text { Adaptations to the home environment } & 134\end{array}$

$\begin{array}{ll}\text { Behavioural change } & 301\end{array}$

Health services $\quad 6$

$\begin{array}{lr}\text { Assistive devices } & 16\end{array}$

$\begin{array}{ll}\text { Total } & 457\end{array}$

$* \mathrm{R} / \mathrm{R}=$ referral / recommendation 


\section{Self-reported compliance with recommendations and referrals}

Figure 2 reports on the net implementation of the referrals and recommendations ensuing from the medical assessments. For 30 of the participants who contacted their GP ( $n=61$ ), the medical assessment resulted in 28 referrals and 14 recommendations. After the implementation period of the programme, 14 participants reported that 8 recommendations and 10 referrals had actually reached them through the GP and had been implemented. For 20 participants who did not contact their GP, the medical assessment resulted in 16 referrals

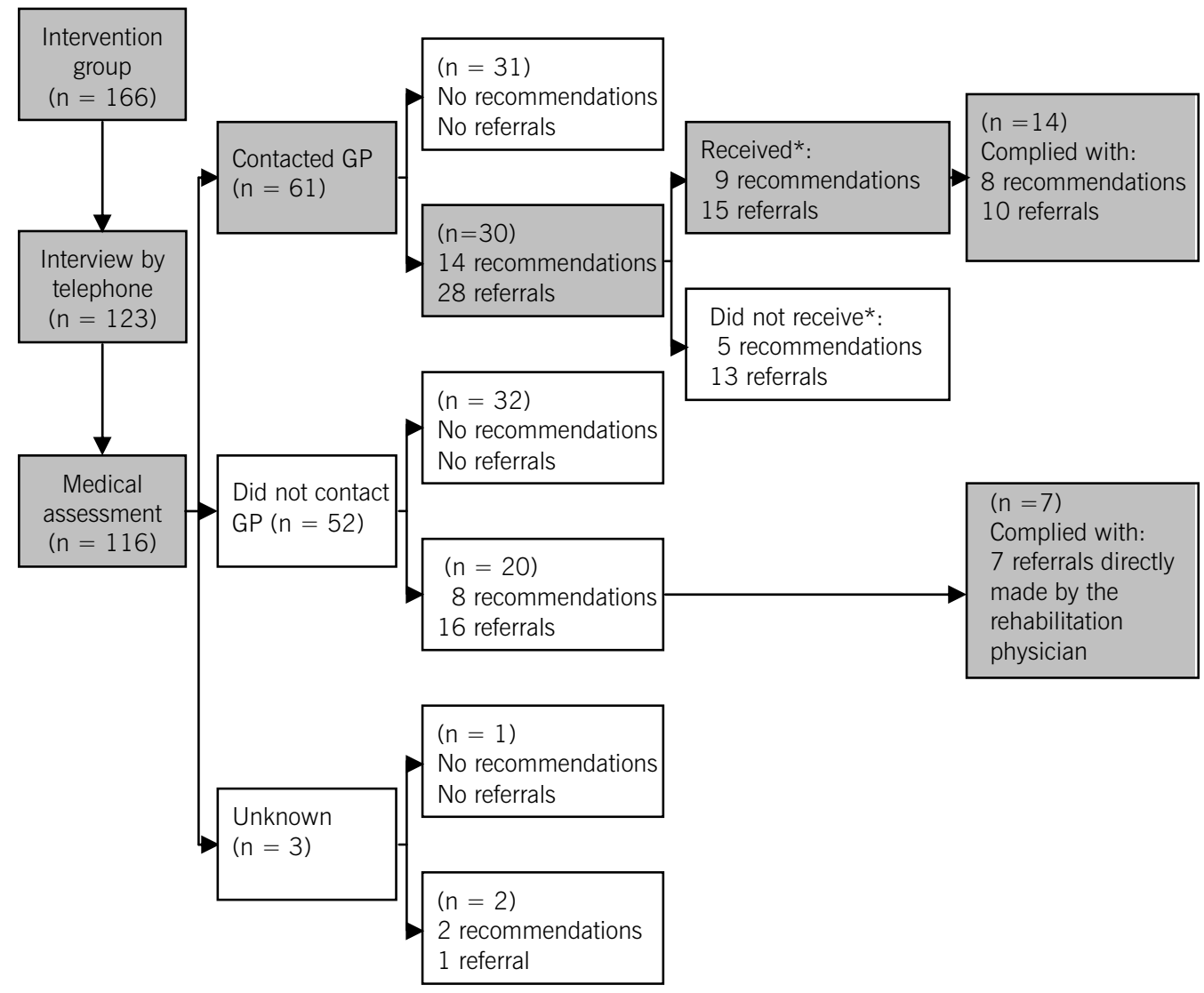

*no numbers of participants are presented due to overlap between categories

(some participants did only receive part of the referrals and/or recommendations).

Figure 2 - Net implementation of the referrals and recommendations ensuing from the medical assessment 
and 8 recommendations. Because these participants did not contact their GPs, these referrals and recommendations did not reach the participants. However, 7 participants complied with the referral to an orthopaedic shoemaker even though none of them had contacted their GP, because the referral was made directly by the rehabilitation physician during the medical assessment.

Figure 3 reports on the net implementation of the recommendations ensuing from the occupational-therapy assessments. A total of 108 participants received 420 recommendations. At the end the implementation period of the programme, 95 of these 108 participants reported that they had received and complied with 249 recommendations.

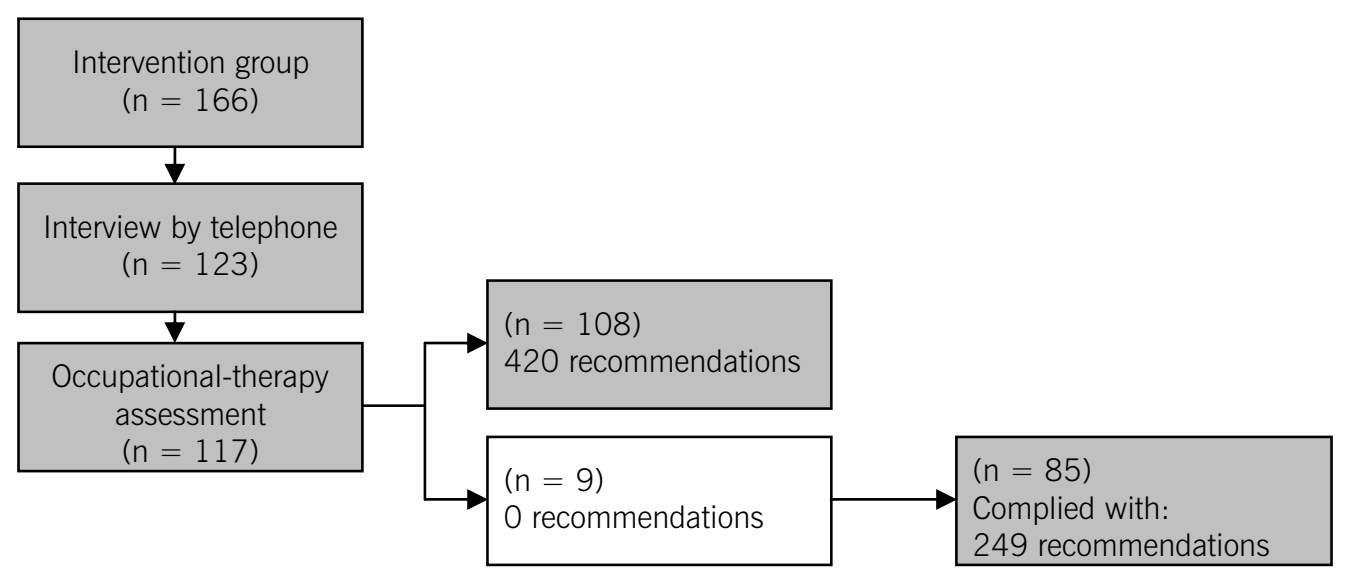

Figure 3 - Net implementation of the recommendations ensuing from the occupational-therapy assessment 
Table 3 shows the results on the nature of the referrals and recommendations ensuing from the medical assessment for those participants who called their GP and the participants' selfreported compliance. As it is not possible to comply with referrals and/or recommendation one did not receive, we calculated the compliance for those participants who actually called their GP and reported that they received referrals and/or recommendations from their GP. Overall, the participants who called their GP and received referrals and/or recommendations complied with 18 out of 24 referrals and recommendations, a compliance of $75 \%$.

Table 3 - Referrals and recommendations resulting from the medical assessment for those participants who called their GP, and participant's self-reported compliance

\begin{tabular}{|c|c|c|c|}
\hline & $\mathrm{R} / \mathrm{R}^{*}$ resulting from & $\mathrm{R} / \mathrm{R}^{*}$ received & Self-reported \\
\hline \multicolumn{4}{|l|}{ Referrals } \\
\hline Cardiologist & 5 & 3 & 3 \\
\hline Osteoporosis examination & 7 & 2 & 1 \\
\hline Orthopaedic shoemaker & 11 & 9 & 6 \\
\hline Orthopaedic instrument maker & 1 & 0 & - \\
\hline Physiotherapist & 2 & 1 & 0 \\
\hline Other referrals & 2 & 0 & - \\
\hline Total referrals & 28 & 15 & 10 \\
\hline \multicolumn{4}{|l|}{ Recommendations } \\
\hline Adjust medication & 3 & 3 & 3 \\
\hline Adjust footwear & 3 & 2 & 1 \\
\hline Further examination & 3 & 0 & - \\
\hline Vitamin B supplementation & 1 & 1 & 1 \\
\hline Other recommendations & 4 & 3 & 3 \\
\hline Total recommendations & 14 & 9 & 8 \\
\hline Total referrals and recommendations from & 42 & 24 & $18(75 \%)$ \\
\hline
\end{tabular}

* $\mathrm{R} / \mathrm{R}=$ referral / recommendation 
Table 4 shows the results on the nature of the recommendations ensuing from the occupational-therapy assessment and the participants' self-reported compliance. Overall, the participants reported having complied with $59 \%$ of the recommendations they had received from the occupational-therapy assessment.

Table 4 - Recommendations ensuing from the occupational-therapy assessment and self-reported compliance with these recommendations

\begin{tabular}{lcc}
\hline Recommendations & \\
Adaptations to the home environment & 124 & $68(55 \%)$ \\
Behavioural change & 279 & $174(62 \%)$ \\
Health services & 6 & $3(50 \%)$ \\
Assistive devices & 11 & $4(36 \%)$ \\
Total & 420 & $249(59 \%)$ \\
\hline
\end{tabular}

${ }^{*} \mathrm{R}=$ recommendation

\section{Participants' and practitioners' opinions about the programme}

\section{Participants' opinions about the programme}

During the telephone interviews, a majority of the participants reported that they had benefited from the assessments. This percentage was $82 \%$ for the medical assessments and $80 \%$ for the occupational-therapy assessments. Overall, $84 \%$ of the participants reported that they had perceived at least some benefit from the programme as a whole. Besides the perceived benefit, the participants were also asked whether they were satisfied with the medical and occupational-therapy assessments. Almost all participants were satisfied, viz. 97\% and 99\% for the medical and occupational-therapy assessments, respectively (ranging from somewhat satisfied to very satisfied). 


\section{Practitioners' opinions}

The practitioners were asked to give their opinion about whether the participants had benefited from the programme. They judged that most participants had benefited, particularly those who received recommendations for footwear, adaptations to the home environment, or assistive devices. In addition, they thought that in most cases the participants were satisfied with the programme. Although the practitioners were optimistic about the programme benefits, they reported that in their opinion a considerable proportion of the participants, i.e. those with only minor health problems, should not have been included in the trial. The practitioners considered it unlikely that these persons would benefit much from the programme.

The practitioners judged the programme to be feasible and considered all aspects included in the assessments relevant. They considered the recording forms to be easy to work with, although some aspects could be improved, such as the structure and layout of the forms. They also mentioned two aspects that should be added to the programme protocol: a preprinted list of medications that increase the risk of falling and an instrument to assess fear of falling.

The practitioners were also positive about their own role in the programme. However, they mentioned that there should be more interdisciplinary consultation and communication between the practitioners to agree on referrals and recommendations. Moreover, both assessments should be more closely tailored to the needs of individual patients and more assessments and training should be done in the home environment. To further optimise the programme, the practitioners recommended redistributing some of the assessment tasks between them, and to do some examinations more thoroughly.

\section{CONCLUSION \& DISCUSSION}

Overall, the programme turned out to be acceptable and feasible for both practitioners and participants. The results of our study show that the programme was largely performed according to protocol. The medical and occupational-therapy assessments led to an average of 3.85 recommendations and/or referrals per participant. However, the number of referrals and recommendations ensuing from the medical assessments was relatively small (on 
average 0.58) compared to the recommendations ensuing from the occupational-therapy assessments (on average 3.57). Participants' self-reported compliance with the advice to contact their GP to be informed of the recommendations and/or referrals from the medical assessment was low to moderate (53\%). Participants who were informed by their GP of the referrals and recommendations reported reasonable to good compliance $(75 \%)$ with these referrals and recommendations. Participants' self-reported compliance with the recommendations they received from the occupational therapists was moderate (59\%). Participants' overall compliance with the recommendations and/or referrals ensuing the medical and occupational-therapy assessments was $60 \%$. Both participants and practitioners judged the programme to be feasible. A large majority of participants reported that they had benefited from the programme.

This process evaluation has provided insight into process-related factors that may explain the lack of effectiveness of our programme. The main process-related factors that may be responsible for the lack of effectiveness are the relatively low numbers of referrals and recommendations ensuing from the medical assessments and participants' poor compliance with the suggestion to contact their GP to be informed of the recommendations and/or referrals resulting from the medical assessment.

The limited number of referrals and recommendations ensuing from the medical assessments may indicate that our study population possibly was relatively healthy and not at high risk for falls and/or already received sufficient medical care. The inclusion criteria of our study and the study of Close et al (16) were comparable, although we additionally excluded participants who were permanently bedridden, fully dependent on a wheelchair, and were not able to complete questionnaires or interviews by phone. Comparison of our population with the population of Close and colleagues(16) revealed that the number of recurrent fallers in our control group was comparable to the control group of Close and colleagues and other studies $(5,16,18,19)$. It is therefore unlikely that differences in population are the only explanation for the limited number of recommendations. It is possible that also differences in regular care in both countries can explain the limited number of recommendations. Possibly regular care in the Netherlands at the time of the study (2002-2005) was better than the regular care in the UK at the time of the study (1995-1998). 
There are various possible explanations for the participants' low compliance with contacting their GP. Participants reported that the most important reasons for not contacting their GP were forgetting to do so, not thinking it useful, and not being aware of the possibility. These reasons may be related to the relatively long period between randomization and the moment the GPs were informed of the results of the assessments (on average 3.5 months). Recommending the participants to contact their GP and sending a subsequent reminder to all participants was apparently not sufficient to stimulate the participants to contact their GP. For our programme, this implies that it is not efficient to let the GPs act as intermediaries between the practitioners doing the assessments and the participants. However, our reason for incorporating the GPs was that we wanted to make the programme fit in easily with regular healthcare. In the Netherlands, referrals to medical specialists are implemented through a patient's GP (20). In addition, GPs are familiar with the health status of their patients and can therefore act as supervisors to provide the best possible care. With hindsight, including GPs in the procedure seems to be an inefficient option, and is likely to have contributed to the lack of effectiveness of our trial. In the British version of the programme, Close and colleagues referred their patients directly to other services or a day hospital for further investigation, assessment or follow-up (16). In the UK, as in the Netherlands, rehabilitation services include examinations, treatment and counselling by medical specialists, paramedical staff and behavioural or rehabilitation therapists. The major advantage of the British day hospital approach is that it produces "a one-stop shop" for patients with complex needs, which would otherwise (like in the Netherlands) require multiple visits to different departments, or multiple visits to GP's, medical specialist and therapists (21).

The present study had some possible limitations. First, participants and practitioners may have given socially desirable answers. We tried to avoid this tendency among participants by gathering data anonymously and by informing them that their answers would not affect their future use of healthcare services. Among practitioners, we tried to avoid social desirable answering by stressing that their comments and recommendations would only be used to improve the programme and not to judge their professionalism. A second limitation of this study is that we did not collect data directly from the GPs. We may have missed relevant data concerning the role of the GPs in the programme, e.g. whether the GP agreed with the suggested referrals and recommendations, and whether the participants actually called them. 


\section{CONCLUSION}

Based on the results of this process evaluation and the lack of effectiveness of our programme we do not recommend implementing the programme in its present form in regular care. We recommend two major adjustments to the programme. Firstly, we recommend to screen the potential participants of the programme on their fall risk by a routinely performed short fall risk screening among patients who attend the A\&E department because of a fall (22-27). Hence it should be possible to discriminate between a low to moderate risk group and a high risk group among community dwelling fallers who attending the A\&E department. Focusing on fallers with a substantially increased risk of recurrent falls may improve the efficiency of the programme. Secondly, we aim to increase the efficiency of the programme by drastically decreasing the time between the patient attending the A\&E department and the implementation of the fall prevention measures. We therefore recommend to perform the medical assessment preferably within two weeks after attending the A\&E department for those directly discharged home, and around discharge for those admitted to hospital after the fall. Furthermore, the occupational-therapy assessment should be performed preferably within two weeks after the patient is being discharged home. To further increase the efficiency, the geriatrician who performs the medical assessment should be permitted to refer patients directly to relevant services in stead of having the GP implement the referrals. The geriatrician and occupational therapist should send the GP a comprehensive report on the outcomes of the assessments and the actions already taken. This would allow the GPs to continue and coordinate the fall prevention measures initiated or implemented by the geriatrician and occupational therapist. A follow-up consultation with the geriatrician and occupational therapist after 6 months is recommended to assess the patient's current risk profile, to increase long-term compliance with fall prevention measures, and to take additional fall prevention measures if necessary. However, whether the recommended adaptations to the programme will be realizable and feasible in Dutch healthcare should be thoroughly explored, because the proposed procedure deviates considerably from usual procedures in the Netherlands. We therefore strongly recommend that both the feasibility and (cost-) effectiveness of this adjusted programme should be studied before implementing it in Dutch regular care. 


\section{ACKNOWLEDGEMENTS AND FUNDING}

We would like to thank the Faculty of Health, Medicine and Life Sciences of Maastricht University, School for Public Health and Primary Care (Caphri), and the Netherlands Organisation for Health Research and Development (ZonMw), Committee on Health Care Efficiency Research Programme, grant 945-02-053, for funding this study. 


\section{REFERENCES}

1. Gillespie $L D$, Gillespie WJ, Robertson MC, Lamb SE, Cumming RG, Rowe BH. Interventions for preventing falls in elderly people. Cochrane Database Syst Rev. 2003(4):CD000340.

2. Chang JT, Morton SC, Rubenstein LZ, Mojica WA, Maglione M, Suttorp MJ, et al. Interventions for the prevention of falls in older adults: systematic review and meta-analysis of randomised clinical trials. Bmj. 2004;328(7441):680.

3. Kannus P, Sievanen H, Palvanen M, Jarvinen T, Parkkari J. Prevention of falls and consequent injuries in elderly people. Lancet. 2005 Nov 26;366(9500):1885-93.

4. Blake AJ, Morgan K, Bendall MJ, Dallosso H, Ebrahim SB, Arie TH, et al. Falls by elderly people at home: prevalence and associated factors. Age Ageing. 1988;17(6):365-72.

5. O'Loughlin J, Robitaille Y, Bolvin J-F, Suissa S. Incidence of and risk factors for falls and injurious falls among the community-dwelling elderly. Am J Epidemiol. 1993;137:342-54.

6. Tinetti ME, Speechley M, Ginter SF. Risk factors for falls among elderly persons living in the community. N Engl J Med. 1988;319(26):1701-7.

7. Nevitt MC, Cummings SR, Hudes ES. Risk factors for injurious falls: a prospective study. J Gerontol. 1991;46(5):M164-70.

8. Fuller GF. Falls in the elderly. Am Fam Physician. 2000;61(7):2159-68, 73-4.

9. Scaf-Klomp W, van Sonderen E, Sanderman R, Ormel J, Kempen GI. Recovery of physical function after limb injuries in independent older people living at home. Age Ageing. 2001 May;30(3):213-9.

10. Yardley L, Donovan-Hall M, Francis K, Todd C. Older people's views of advice about falls prevention: a qualitative study. Health Educ Res. 2006 Aug;21(4):508-17.

11. Vellas BJ, Wayne SJ, Romero LJ, Baumgartner RN, Garry PJ. Fear of falling and restriction of mobility in elderly fallers. Age Ageing. 1997;26:189-93.

12. Bloem BR, Boers, I., Cramer, M., Westendorp, R.G.J., Gerschlager, W. Falls in the elderly: identification of risk factors. Wiener Klinische Wochenschrift. 2001;113:352-62.

13. Kiel DP, O'Sullivan P, Teno JM, Mor V. Health care utilization and functional status in the aged following a fall. Med Care. 1991;29(3):221-8.

14. American Geriatrics Society BGS, and American Academy of Orthopaedic Surgeons Panel on Falls Prevention. Guideline for the prevention of falls in older persons. J Am Geriatr Soc. 2001;49(5):664-72.

15. Davison J, Bond J, Dawson P, Steen IN, Kenny RA. Patients with recurrent falls attending Accident \& Emergency benefit from multifactorial intervention--a randomised controlled trial. Age Ageing. 2005 March 1, 2005;34(2):162-8. 
16. Close J, Ellis M, Hooper R, Glucksman E, Jackson S, Swift C. Prevention of falls in the elderly trial (PROFET): a randomised controlled trial. Lancet. 1999 Jan 9;353(9147):93-7.

17. Hendriks MR, van Haastregt JC, Diederiks JP, Evers SM, Crebolder HF, van Eijk JT. Effectiveness and costeffectiveness of a multidisciplinary intervention programme to prevent new falls and functional decline among elderly persons at risk: design of a replicated randomised controlled trial [ISRCTN64716113]. BMC Public Health. $2005 \operatorname{Jan} 14 ; 5: 6$.

18. Hendriks MR, Bleijlevens MH, van Haastregt JC, Crebolder HF, Diederiks JP, Evers SM, et al. Lack of effectiveness of a multidisciplinary fall-prevention program in elderly people at risk: a randomized, controlled trial. J Am Geriatr Soc. 2008 Aug;56(8):1390-7.

19. Tinetti ME, Baker DI, McAvay G, Claus EB, Garrett P, Gottschalk M, et al. A multifactorial intervention to reduce the risk of falling among elderly people living in the community. N Eng/ J Med. 1994;331(13):821-7.

20. Kulu Glasgow I, Delnoij D, de Bakker D. Self-referral in a gatekeeping system: patients' reasons for skipping the general-practitioner. Health policy. 1998 Sep;45(3):221-38.

21. Black DA. The geriatric day hospital. Age Ageing. 2005 Sep;34(5):427-9.

22. Stalenhoef PA, Diederiks JP, Knottnerus JA, Kester AD, Crebolder HF. A risk model for the prediction of recurrent falls in community-dwelling elderly: a prospective cohort study. J Clin Epidemiol. 2002 Nov;55(11):1088-94.

23. Stel VS, Pluijm SM, Deeg DJ, Smit JH, Bouter LM, Lips P. A classification tree for predicting recurrent falling in community-dwelling older persons. J Am Geriatr Soc. 2003 Oct;51(10):1356-64.

24. Pluijm SM, Smit JH, Tromp EA, Stel VS, Deeg DJ, Bouter $L M$, et al. A risk profile for identifying community-dwelling elderly with a high risk of recurrent falling: results of a 3-year prospective study. Osteoporos Int. 2006;17(3):417-25.

25. Tromp AM, Pluijm SM, Smit JH, Deeg DJ, Bouter LM, Lips P. Fall-risk screening test: a prospective study on predictors for falls in community-dwelling elderly. J Clin Epidemiol. 2001 Aug;54(8):837-44.

26. Vassallo M, Stockdale R, Sharma JC, Briggs $R$, Allen S. A comparative study of the use of four fall risk assessment tools on acute medical wards. J Am Geriatr Soc. 2005 Jun;53(6):1034-8.

27. Kalula SZ, de Villiers L, Ross K, Ferreira M. Management of older patients presenting after a fall--an accident and emergency department audit. S Afr Med J. 2006 Aug;96(8):718-21. 


\section{LESSONS LEARNED FROM A MULTIDISCIPLINARY FALL PREVENTION PROGRAMME: THE OCCUPATIONAL-THERAPY PART}

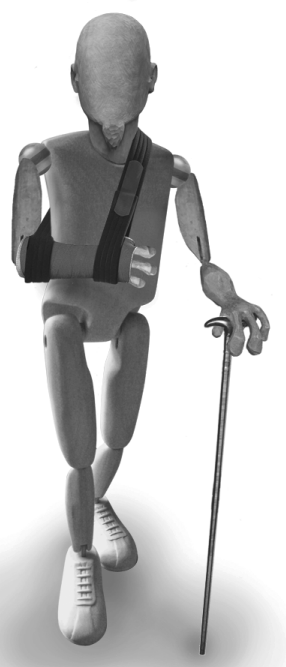

Michel HC Bleijlevens

Marike RC Hendriks Jolanda CM van Haastregt Harry FJM Crebolder Jacques ThM van Eijk 


\section{ABSTRACT}

\section{Objective}

To gain insight into the contribution of the occupational-therapy part of a multidisciplinary fall prevention programme towards the reduction of falls and functional decline.

\section{Design}

A descriptive and exploratory study

\section{Methods}

Data were collected in the context of a randomised controlled trial that found no effect of a multidisciplinary fall prevention programme. The study population comprised 166 participants, two occupational therapists (OTs), and one official from each of the five participating municipalities. We collected data on the recommendations arising from the OT part of the programme, the extent to which those recommendations were implemented and what OTs did to stimulate implementation of behaviour change.

\section{Results}

The occupational-therapy programme resulted in 457 recommendations; $65 \%$ of the recommendations regarding services and assistive devices were implemented. It took on average six months to implement recommended home modifications. Advice about behaviour change predominantly comprised recommendations to reduce risky behaviour.

\section{Conclusion}

To improve the occupational-therapy programme we suggest more rapid implementation of recommendations. Second, participants should be supported to achieve recommended changes. Furthermore, the occupational therapists should use theory-based techniques to stimulate behaviour change and use follow-up visits to promote maintenance of the desired behaviour. 


\section{INTRODUCTION}

Falls and their consequences have been recognised as a great strain on the well-being of older people. Each year, approximately $30 \%$ of people aged 65 years and older who are living in the community sustain a fall (1-5). Several studies have shown that the occurrence of falls is associated not only with intrinsic factors but also with extrinsic factors such as environmental hazards (6-11). It thus seems sensible to include home assessments followed by environmental modifications in fall prevention programmes (12). However, there is no clear evidence for the effectiveness of home assessment and modification in preventing falls (3, 13-19). Despite the ambiguous evidence, assessing and addressing environmental hazards has been embedded in numerous multifaceted fall prevention programmes for elderly people living in the community (1, 14-18, 20-22). Lord and colleagues (19) stated in their review that home hazard reduction is an effective strategy to prevent falls, provided it is aimed at older people with a history of falls and mobility impairments, but not for the general population of elderly people. They also stated that the effectiveness of home hazard modification depends mainly on behaviour change.

Recently, we evaluated the effectiveness of a multidisciplinary fall prevention programme in the Netherlands in a randomised controlled trial (RCT) (1). We assessed whether this programme was more effective than usual care in preventing falls and functional decline in community-dwelling elderly people who attended an accident and emergency department (A\&E department) after a fall. The programme consisted of a medical and occupationaltherapy assessment, followed by recommendations and further referral if indicated. The medical part of the programme consisted of a detailed medical risk assessment followed by recommendations and referrals (23). The occupational-therapy part consisted of a functional and environmental risk assessment resulting in recommendations in terms of services and assistive devices, and advice about behaviour change (23). The programme turned out not to be effective in preventing falls and functional decline (1). The results of a process evaluation which was performed alongside the trial (24) showed that the programme was considered feasible and acceptable by both participants and practitioners. The medical part of the programme resulted in relatively few recommendations (on average less than one per participant). In addition, a substantial part of these recommendations never reached the 
patient because $45 \%$ of the participants did not comply with the recommendation to contact their GP to discuss the results of the examinations. Hence, it is not surprising that this part of the programme failed to have a favourable effect on falls and daily functioning.

In contrast to the medical part of the programme, however, the occupational-therapy part resulted in a substantial number of referrals and recommendations (on average more than 3 per participant), which were directly communicated to the participants by the occupational therapists. Considering the number of recommendations and the fact that these reached the participants directly, it remains unclear why the programme, overall, did not lead to a reduction of falls and functional decline. Therefore, the role of the occupational-therapy part of the programme in preventing new falls and functional decline should be further examined.

In order to gain insight into the contribution of the occupational-therapy programme towards the reduction in falls and functional decline, this paper aims to examine (a) the number and nature of the recommendations ensuing from the occupational-therapy part of the programme, (b) the extent to which the recommendations with regard to services and assistive devices were actually implemented and (c) what was done by the occupational therapists to stimulate the implementation of the recommended behaviour changes.

\section{METHODS}

\section{Design}

The current paper reports on a descriptive and exploratory study in which both quantitative and qualitative data were gathered. The Medical Ethics Committee of Maastricht University/ University Hospital Maastricht approved the study. All participants signed an informed consent form.

\section{Occupational-therapy programme}

The occupational-therapy programme was provided by an occupational therapist at the participants' homes and comprised a functional and environmental evaluation to identify risk factors for new falls (23). Daily functioning was assessed by the 15-item Frenchay Activity 
Index (FAl) (25) and an occupational-therapy checklist (26, 27). In addition, the Falls Handicap Inventory (FHI) (28) was used to assess handicaps associated with repeated falls. Environmental hazards in and around the participants' homes were identified and recorded by means of a home-safety checklist (29).

The functional and environmental assessments resulted in recommendations for services and assistive devices, and instructions for behaviour change. These recommendations were given to the participants by the occupational therapists during the home visits. Afterwards, the participants received a letter with the recommendations by way of reminder. A copy of this letter was sent to the participant's GP to inform him/her of the results of the occupational therapy programme.

At the time of the study, some of the recommended services and assistive devices were provided under the Services for the Disabled Act (WVG) which was implemented by the municipal authorities (30). The occupational therapists administering the occupationaltherapy programme were authorised to advise the five municipalities entrusted with the implementation of the Act in the study region about the care needed. Subsequently, the municipal authorities decided whether or not to fund the recommended services and assistive devices. After a favourable decision from the municipal authorities had been received, the service and/or device could be provided.

Certain other recommended assistive devices, such as rollators and canes, had to be purchased by the participants themselves, but could often be partly or wholly refunded by their health insurance company.

\section{Study population}

The study population was derived from that of an RCT assessing the effectiveness and costeffectiveness of our multidisciplinary fall prevention programme (1) and comprised 166 older people, aged 65 years or over, who had attended the A\&E department and/or the outof-hours GP service offered at the hospital as a result of a fall. The study population also included the two occupational therapists who administered the programme as part of their normal working routine and one official from each of the five participating municipalities who was entrusted with the implementation of the WVG act. 


\section{Measurements}

\section{Number and nature of recommendations}

We recorded the number and nature of recommendations ensuing from the occupationaltherapy programme by collecting data from specially designed forms completed by the occupational therapists during the home visits.

\section{Implementation of recommendations for services and assistive devices provided under the WVG act.}

In order to examine the actual implementation of the recommendations with regard to services and assistive devices provided under the Services for the Disabled Act (WVG), we analysed the municipal authorities' existing implementation records. In addition, we recorded the time interval between the occupational-therapy assessment and the implementation of the ensuing recommendations. We used structured recording forms to collect data on the actual implementation and the time interval.

\section{Advice on behaviour change}

This information was gathered by means of structured recording forms completed by the therapists, as well as by structured face-to-face in-depth interviews with the occupational therapists and a plenary group discussion with all practitioners involved in the multidisciplinary fall prevention programme and the research team.

\section{Data analysis}

Quantitative data from the questionnaires and recording forms were analysed by means of descriptive statistics. All analyses were performed in SPSS 14.0. From both the in-depth interviews with the occupational therapists and the plenary group discussion minutes were taken. Based on the written reports of the in-depth interviews and the plenary group discussion, answers were thematically categorised and summarised. One researcher (MB) independently reviewed the answers given. In case of doubt a second researcher was consulted (MH). 


\section{RESULTS}

\section{Participation}

Of the 166 people included in this study, 28 did not undergo any part of the multidisciplinary fall prevention programme, because they withdrew from the study before the fall prevention programme started or had problems scheduling in the medical and occupational-therapy parts of the programme. Reasons for withdrawal were: death $(n=2)$; refusal to continue participation $(n=8)$; health problems $(n=12$; and other reasons $(n=5)$. Another ten participants underwent only the medical part of the programme, because of personal reasons $(n=6)$, or withdrew from the study before the occupational-therapy programme took place $(n=4)$. The remaining 128 participants (77\%) underwent the occupational-therapy programme. Of these 128 participants, 11 withdrew from the study after completing the programme. Reasons for withdrawal were: death $(n=3)$; refusal to continue participation $(n=4)$; health problems $(n=4)$.

\section{Number and nature of recommendations}

The occupational-therapy programme resulted in a total of 457 recommendations for the 128 participants (table 1 ), which is on average 3.6 recommendations per participant. These recommendations can be subdivided into three main categories: (1) Services and assistive devices provided under the Services for the Disabled Act (WVG); (2) assistive devices individually purchased; and (3) advice on behaviour change. Since 6 recommendations did not fit these three main categories, a fourth category was added, viz. referrals to other health services. Overall, about two thirds of all recommendations concerned instructions for behaviour change. In this category, almost half of the recommendations (46\%) were related to the correct use of home adaptations and assistive devices. The category of services and assistive devices provided under the WVG act accounted for $29 \%$ of the recommendations made by the occupational therapists. This category can be subdivided into home adaptations, assistive devices and moving house (table 1). Four percent of the recommendations concerned the category of assistive devices individually purchased, and the smallest category (1\%) comprised recommendations for referrals to other health services (e.g. homes for the elderly). 
Table 1 - Recommendations resulting from the occupational-therapy programme

Number of recommendations

Services and devices provided under the WVG act

Home adaptations

Installing hand rails

Constructional adaptations

Assistive devices

Shower chair

Chair lift

Raised toilet

Transfer bench

Bath lift

Toilet chair

Mobility scooter

5

Move house ${ }^{1}$

Total

Assistive devices not provided under the WVG act

Lift chair

Ergonomic work chair

Ankle-foot orthosis

Cane

Rollator

High/Low bed

Antiskid mat

Total

Instructions about behaviour change

Use assistive devices

Adjust working pace

Remove loose rugs / furniture

Increase self-confidence

Avoid dangerous situations

Use home adaptations

Total

Health services

Intake for admission to home for the elderly Intake for admission to sheltered accommodation

Consultation with General Practitioner

${ }^{1}$ If the recommended home adaptations were too expensive or were not feasible, participants received a recommendation to move to a specially adapted house. This was partly refunded under the WVG act. 


\section{Implementation of recommendations for services and assistive devices provided under the WVG act}

A total of 46 participants received 123 recommendations (2.7 per participant) with regard to services and assistive devices provided under the WVG act. After the assessment, 36 participants applied for 111 services for daily living and/or assistive devices. The other 10 participants did not apply to the municipal authorities for any services or assistive devices, but five of them implemented the recommendations themselves.

Of the 111 applications for services and assistive devices, 93 were approved by the municipal authorities. The officials of the five municipalities reported that 80 of the 93 approved recommendations for services for daily living and/or assistive devices had actually been implemented (Table 2).

Table 2 - Implementation of recommendations for services and assistive devices provided under the WVG act

\begin{tabular}{lcccc}
\hline $\begin{array}{c}\text { Number of } \\
\text { recommendations } \\
\text { ensuing from the } \\
\text { occupational-therapy } \\
\text { programme }\end{array}$ & $\begin{array}{c}\text { Number of } \\
\text { recommendations } \\
\text { applied for by the } \\
\text { participants }\end{array}$ & $\begin{array}{c}\text { Number of } \\
\text { recommendations } \\
\text { accepted by the } \\
\text { municipal }\end{array}$ & $\begin{array}{c}\text { Number of } \\
\text { recommendations } \\
\text { implemented } \\
\text { according to }\end{array}$ \\
\hline Home adaptations & 90 & 82 & 71 & municipal authorities \\
Assistive devices & 23 & 21 & 18 & 62 \\
Moving house & 10 & 8 & 4 & 2 \\
Total & 123 & 111 & 93 & 80 \\
\hline
\end{tabular}


We assessed the time interval between the occupational therapy programme and the implementation of the recommendations for home adaptations, using information provided by the municipal authorities. The average time interval between recommendations for home adaptations and their implementation was 6.2 months, whereas the intervals for recommendations for assistive devices and recommendations to move to other accommodation were 5.3 and 9.2 months, respectively. Of the 46 individuals who applied for a service or assistive device under the WVG act, 28 sustained a fall during one year of follow-up. However, only one of these people fell after the recommended recommendations had been implemented: 10 people had already fallen before they took part in the occupational-therapy programme and 17 people fell after they had received the programme, but before the recommendations had been implemented.

\section{Advice on behaviour change}

The structured recording forms, the structured in-depth interview with the occupational therapists and the plenary group discussion revealed that the therapists did not use theorybased strategies to promote behaviour change to reduce the risk of falls. The occupational therapists instructed the participants on how to change their risky behaviour, but the participants were not supported any further in order to achieve the recommended behaviour change. The occupational therapists indicated that they had serious doubts whether the instructions given were sufficient to achieve a lasting behaviour change. There were no follow-up visits to check whether the recommendations had actually been implemented, nor booster sessions to focus the participant's attention on the recommended changes again.

\section{DISCUSSION AND CONCLUSION}

The recommendations resulting from the occupational-therapy assessment can be divided into four main categories: (a) advice on behaviour change (66\%); (b) services and assistive devices provided under the Services for the Disabled Act (WVG) (29\%); (c) assistive devices individually purchased (4\%); and (d) referral to other health services (1\%). Advice on behaviour change was predominantly confined to recommendations to reduce risky behaviour, made during the home visit by the occupational therapists, but these were not supported by follow-up sessions. Of the recommendations regarding services and assistive devices covered 
by the WVG act, $65 \%$ were actually implemented, as reported by the municipal authorities. In view of the number of recommendations and the fact that these were directly communicated to participants, it remains unclear why this part of the programme did not significantly contribute to an overall reduction in falls and functional decline. Our in-depth analysis of the occupational-therapy programme furnished a number of possible explanations for the fact that the programme did not contribute to a reduction of falls and functional decline during the 12 months of follow-up. First, the ineffectiveness may be explained by the fact that $35 \%$ of the recommendations were not implemented during the follow-up period. However, as we reported in a previous paper (24), compliance with the recommendations was reasonable and comparable to the compliance rates reported by other studies in this domain $(15,17,31,32)$.

Second, the implementation of recommendations for services and assistive devices provided under the WVG act took almost six months. Of those who sustained another fall during the one year of follow-up $(n=28)$, all but one $(96 \%)$ fell before their WVG applications had been implemented. This suggests that it is very important to decrease the time that elapses between recommendation and implementation.

Third, Lord and colleagues reported that home hazard reduction is an effective fall prevention strategy if targeted at older people with a history of falls and mobility limitations (19). Our study population may not have met these criteria. Although we included persons who had recently experienced an injurious fall (and were thus considered to be at increased risk for recurrent falls) the occupational therapists stated that the people visited were relatively healthy and had, on average, few mobility impairments. This is supported by the participants' mean score on the Groningen Activity Restriction Scale (GARS), which measures activities of daily living (ADL) and instrumental activities of daily living (IADL) disabilities (33). The mean score ( \pm SD) for the 128 persons who took part in the occupational therapy programme was 17.5 ( \pm 7.06$)$ on a scale ranging from 11-44, where a low score indicates few or no limitations in terms of ADL and IADL.

Fourth, the occupational therapy programme resulted in 301 recommendations with regard to behaviour change. However, this aspect of the programme was limited to pointing out a person's fall-related risk behaviour during one home visit and suggesting a change in behaviour to reduce their fall risk in the future. It is doubtful whether this single contact is sufficient to result in the recommended behaviour change (34). 


\section{Lessons learned / Recommendations}

Based on the results of this study and the results reported in the previously published papers on the effects and feasibility of our multidisciplinary fall prevention programme $(1,24)$, we conclude that the occupational-therapy part of the programme should not be implemented in its current form in regular care.

Our findings suggest a number of recommendations to improve the programme. First, we should aim to increase the efficiency of the programme by drastically reducing the time between the occupational-therapy programme and the actual implementation of recommendations for services and assistive devices provided under the WVG act. Second, to increase compliance with the recommendations, participants should be supported over a period of time to achieve the recommended changes. This could include follow-up visits to check whether the services and assistive devices were actually implemented and were being correctly used. Furthermore, the occupational therapists should use theory-based techniques to stimulate behaviour change and use follow-up visits to encourage behaviour change and promote maintenance of the desired behaviour.

Finally, in order to recruit a population likely to derive most benefit from the programme, there should be a more stringent selection procedure. Participants should have a history of recurrent falls and moderate to severe mobility impairments.

\section{ACKNOWLEDGEMENTS}

We would like to thank the Faculty of Health, Medicine and Life Sciences of Maastricht University, School for Public Health and Primary Care (Caphri), and the Netherlands Organisation for Health Research and Development (ZonMw), Committee on Health Care Efficiency Research Programme, grant 945-02-053, for funding this study. In addition, we would like to thank Charlotte H. Mom-Schuurman for her contribution (CIZ Centre healthcare indications in the Netherlands). 


\section{REFERENCES}

1. Hendriks MR, Bleijlevens MH, van Haastregt JC, Crebolder HF, Diederiks JP, Evers SM, et al. Lack of effectiveness of a multidisciplinary fall-prevention program in elderly people at risk: a randomized, controlled trial. J Am Geriatr Soc. 2008 Aug;56(8):1390-7.

2. Gillespie LD, Gillespie WJ, Robertson MC, Lamb SE, Cumming RG, Rowe BH. Interventions for preventing falls in elderly people. Cochrane Database Syst Rev. 2003(4):CD000340.

3. Chang JT, Morton SC, Rubenstein LZ, Mojica WA, Maglione M, Suttorp MJ, et al. Interventions for the prevention of falls in older adults: systematic review and meta-analysis of randomised clinical trials. Bmj. 2004;328(7441):680.

4. Kannus P, Sievanen H, Palvanen M, Jarvinen T, Parkkari J. Prevention of falls and consequent injuries in elderly people. Lancet. 2005 Nov 26;366(9500):1885-93.

5. McClure R, Turner C, Peel N, Spinks A, Eakin E, Hughes K. Population-based interventions for the prevention of fall-related injuries in older people. Cochrane Database Syst Rev. 2005(1):CD004441.

6. Wyman JF, Croghan CF, Nachreiner NM, Gross CR, Hatch Stock H, Talley K, et al. Effectiveness of Education and Individualized Counseling in Reducing Environmental Hazards in the Homes of Community-Dwelling Older Women. J Am Geriatr Soc. 2007;55:1548-56.

7. Lach HW, Reed, T., Arfken, C.L., Miller, J.P., Paige, G.D. Falls in the elderly: reliability of a classification system. Journal of the American Geriatrics Society. 1991;39:197-202.

8. Hill K, Schwarz J, Flicker L, Carroll S. Falls among healthy, community-dwelling, older women: a prospective study of frequency, circumstances, consequences, and prediction accuracy. Australian and New Zealand Journal of Public Health. 1999;23:41-8.

9. Mackenzie L, Byles J, Higginbotham N. A prospective community-based study of falls among older people in Australia: frequency, circumstances, and consequences. OTJR. 2002;22(4):143-52.

10. Masud T, Morris, R.O. Epidemiology of falls. Age Ageing. 2001;30 (S4):3-7.

11. Berg WP, Alessio HM, Mills EM, Tong C. Circumstances and consequences of falls in independent community-dwelling older adults. Age Ageing. 1997;26(4):261-8.

12. Cumming RG, Thomas M, Szonyi G, Salkeld G, O'Neill E, Westbury C, et al. Home visits by an occupational therapist for assessment and modification of environmental hazards: a randomized trial of falls prevention. $J$ Am Geriatr Soc. 1999;47(12):1397-402.

13. American Geriatrics Society BGS, and American Academy of Orthopaedic Surgeons Panel on Falls Prevention. Guideline for the prevention of falls in older persons. J Am Geriatr Soc. 2001;49(5):664-72. 
14. Davison J, Bond J, Dawson P, Steen IN, Kenny RA. Patients with recurrent falls attending Accident \& Emergency benefit from multifactorial intervention--a randomised controlled trial. Age Ageing. 2005 March 1, 2005;34(2):162-8.

15. Day L, Fildes B, Gordon I, Fitzharris M, Flamer H, Lord S. Randomised factorial trial of falls prevention among older people living in their own homes. Bmj. 2002;325(7356):128.

16. Pardessus V, Puisieux F, Di Pompeo C, Gaudefroy C, Thevenon A, Dewailly P. Benefits of home visits for falls and autonomy in the elderly: a randomized trial study. Am J Phys Med Rehabil. 2002;81(4):247-52.

17. Nikolaus T, Bach M. Preventing Falls in Community-Dwelling Frail Older People Using a Home Intervention Team (HIT): Results From the Randomized Falls-HIT Trial. J Am Geriatr Soc. 2003;51:300-5.

18. Close J, Ellis M, Hooper R, Glucksman E, Jackson S, Swift C. Prevention of falls in the elderly trial (PROFET): a randomised controlled trial. Lancet. 1999 Jan 9;353(9147):93-7.

19. Lord SR, Menz HB, Sherrington C. Home environment risk factors for falls in older people and the efficacy of home modifications. Age Ageing. 2006 Sep;35 Suppl 2:ii55-iï.

20. Wijlhuizen GJ, du Bois P, van Dommelen P, Hopman-Rock M. Effect evaluation of a multifactor community intervention to reduce falls among older persons. Int J Inj Contr Saf Promot. 2007 Mar; 14(1):25-33.

21. Sjösten N, Salonoja M, Piirtola M, Vahlberg $T$, Isoaho R, Hyttinen $H$, et al. A multifactorial fall prevention programme in home-dwelling elderly people: A rondomized-controlled trial. Public Health. 2007; 121:308-18.

22. La Grow SJ, Robertson MC, Campbell AJ, Clarke GA, Kerse NM. Reducing hazard related falls in people 75 years and older with significant visual impairment: how did a successful program work? Inj Prev. 2006; 12:296-301.

23. Hendriks MR, van Haastregt JC, Diederiks JP, Evers SM, Crebolder HF, van Eijk JT. Effectiveness and costeffectiveness of a multidisciplinary intervention programme to prevent new falls and functional decline among elderly persons at risk: design of a replicated randomised controlled trial [ISRCTN64716113]. BMC Public Health. 2005 Jan 14;5:6.

24. Bleijlevens MH, Hendriks MR, van Haastregt JC, van Rossum E, Kempen GI, Diederiks JP, et al. Process factors explaining the ineffectiveness of a multidisciplinary fall prevention programme: a process evaluation. BMC Public Health. 2008 Sep 24;8(1):332.

25. Schuling J, de Haan R, Limburg M, Groenier KH. The Frenchay Activities Index. Assessment of functional status in stroke patients. Stroke. 1993 Aug;24(8):1173-7.

26. Rooken M, Van der Ven S. Standaard voor het afleggen van een huisbezoek door ergotherapeuten: Stroomschema en formulier. Utrecht: Nederlandse Vereniging voor Ergotherapie; 2001. 
27. Ummels C, Lahaye V. Ergotherapeutische registratielijst voor een ADL-zelf-verzorgingsobservatie: Handleiding en formulier. Utrecht: Nederlandse Vereniging voor Ergotherapie; 2001.

28. Rai GS, Kiniorns M, Wientjes H. Falls Handicap Inventory (FHI)--an instrument to measure handicaps associated with repeated falls. J Am Geriatr Soc. 1995;43(6):723-4.

29. Stalenhoef P, Diederiks J, Knottnerus A, de Witte L, Crebolder H. How predictive is a home-safety checklist for indoor fall risk in the elderly living in the community? Eur J Gen Pract. 1998;4:114-20.

30. Jedeloo S, De Witte LP, Linssen BA, Schrijvers AJ. Client satisfaction with service delivery of assistive technology for outdoor mobility. Disabil Rehabil. 2002 Jul 10;24(10):550-7.

31. Stevens M, Holman CD, Bennett N. Preventing falls in older people: impact of an intervention to reduce environmental hazards in the home. J Am Geriatr Soc. 2001 Nov;49(11):1442-7.

32. Cumming RG, Thomas M, Szonyi G, Frampton G, Salkeld G, Clemson L. Adherence to occupational therapist recommendations for home modifications for falls prevention. Am J Occup Ther. 2001 NovDec;55(6):641-8.

33. Kempen GI, Miedema I, Ormel J, Molenaar W. The assessment of disability with the Groningen Activity Restriction Scale. Conceptual framework and psychometric properties. Soc Sci Med. 1996 Dec;43(11):1601-10.

34. Gates S, Lamb SE, Fisher JD, Cooke MW, Carter YH. Multifactorial assessment and targeted intervention for preventing falls and injuries among older people in community and emergency care settings: systematic review and meta-analysis. Bmj. 2008 Jan 19;336(7636):130-3. 



\section{INJURIOUS FALLS: RELATIONSHIP BETWEEN LOCATION AND ACTIVITY}

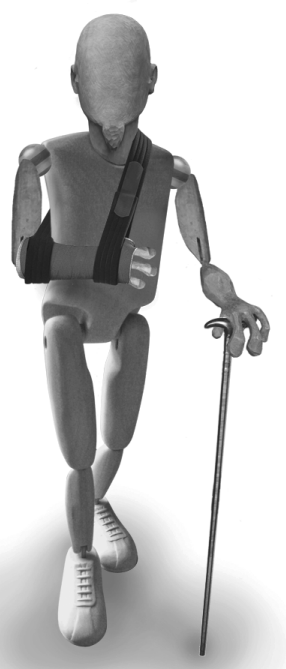

Michel HC Bleijlevens

Joseph PM Diederiks

Marike RC Hendriks Jolanda CM van Haastregt Harry FJM Crebolder Jacques ThM van Eijk 


\section{ABSTRACT}

\section{Objective}

We assessed whether older persons who sustain an injurious fall can be classified into specific fall types, based on a combination of fall location and activity up to the moment of the fall. In addition, we assessed whether specific injurious fall types are related to causes of the fall, consequences of the fall, socio-demographic characteristics, and health-related characteristics.

\section{Design}

An exploratory, cross-sectional study.

\section{Setting}

Accident \& Emergency department at a University Hospital, the Netherlands

\section{Participants}

333 community-dwelling Dutch elderly people aged 65 years or over who attended an accident and emergency department after a fall.

\section{Measurements}

All participants received a self-administered questionnaire after being discharged home. The questionnaire comprised items concerning circumstances of the injurious fall, causes of the fall, consequences of the fall, socio-demographic characteristics and health-related characteristics.

\section{Results}

We identified 4 injurious fall types: 1) Indoor falls related to lavatory visits (hall and bathroom); 2) Indoor falls during other activities of daily living; 3) Outdoor falls near the home during instrumental activities of daily living; 4) Outdoor falls away from home, occurring during walking, cycling, and shopping for groceries. These injurious fall types were significantly related to age, cause of the fall, activity avoidance and daily functioning.

\section{Conclusion}

Our data suggests that in case of a faller ( $<80$ year) who has fallen outside and a faller ( $\geq 80$ year) who has fallen inside we should have special attention for extrinsic causes and intrinsic causes respectively. However, the conclusions reached in this exploratory analysis are tentative and need to be validated in a separate dataset. 


\section{BACKGROUND}

Falls and fall-related injuries in the elderly constitute a significant problem for individuals as well as for society. One out of three elderly persons aged 65 years or older falls at least once a year (1-3). In half of all cases, a fall results in some kind of physical injury (4-6). Approximately $5 \%$ of all falls in community-dwelling elderly people result in a fracture. Another 5 to $10 \%$ of falls result in serious soft tissue injury, such as severe head injury and joint dislocations (3, 4, 7-12). In addition, falls can have considerable psychosocial consequences, like fear of falling, activity avoidance, and social isolation $(13,14)$.

Falls resulting in injuries require special attention, since these falls are responsible for increased levels of healthcare utilization and consequent costs $(6,15-20)$. Unless we undertake effective preventive measures, the societal and economic burden of falls and fallrelated injuries will increase in the coming decades as a result of the growing number of aged people. It therefore seems important to develop fall prevention measures to reduce injurious falls.

In recent decades, many interventions have been developed to prevent falls in older persons (21). Prevention programmes comprising multidisciplinary and multifactorial interventions that screen for health and environmental risk factors and address these factors are expected to be particularly effective in preventing falls (1-3, 21-24). Nevertheless, systematic reviews provide only modest benefit of multifactorial programmes in preventing falls (13, 22-24). Interventions to prevent fall-induced injuries, often aim to reduce the risk of fractures by taking single intervention measures like regular exercise, intake of nutritional supplements (calcium, vitamin D) or the use of hip protectors $(3,22)$. However, evidence for the effectiveness of these interventions is even more limited $(3,22)$. Therefore, we need to search for additional strategies to improve the effectiveness of these interventions. We should especially think of strategies to ensure less fall-related injuries if a fall does occur. For example, it may be useful to use energy-absorbent surfaces in high risk locations and hip protectors in order to decrease the impact of a fall. We may also think of the use of hip protectors., However, to be able to do this, we need insight in the circumstances of injurious falls. Knowledge about the circumstances under which injurious falls occur could provide 
healthcare workers with better tools to prevent falls and fall-related injuries. Several studies already reported on circumstances under which falls occur, such as the location of the fall and the activity the person was engaged in up to the moment of the fall, but did not the joint presence of these circumstances $(9,25-30)$. Therefore, the present study aims to answer the following questions:

1. Is it possible to establish a classification of injurious fall types based on fall location and activity up to the moment of the fall?

2. What is the relationship between injurious fall types on the one hand and sociodemographic characteristics, causes of the fall, consequences of the fall, and healthrelated characteristics on the other?

\section{METHODS}

\section{Design, participants, and setting}

We carried out an exploratory, cross-sectional study to identify injurious fall types based on location of the fall and activity up to the moment of the fall. The population of this study was derived from a randomised controlled trial (RCT) assessing the effectiveness and costeffectiveness of a multidisciplinary fall prevention programme (31). Injurious falls were defined as falls resulting in some kind of physical injury for which persons attended the Accident \& Emergency (A\&E) department. The study design and protocols were approved by the Medical Ethics Committee of Maastricht University and the University Hospital Maastricht. Eligible persons were community-dwelling elderly people aged 65 years and over living in Maastricht (the Netherlands) or its surrounding area. All persons had visited the A\&E department at the University Hospital Maastricht (which includes an out-of-hours GP service) for the consequences of a fall. Eligible persons were excluded if they were unable to communicate in Dutch, unable to complete questionnaires or interviews by telephone, cognitively impaired (a score of less than 4 on the Abbreviated Mental Test 4), admitted to a hospital or other institution for more than four weeks from the date of inclusion, permanently bedridden or fully dependent on a wheelchair. A total of 333 persons were included in the present study. 


\section{Measurements}

All participants received a self-administered questionnaire after being discharged home (i.e. immediately after treatment of the injuries resulting from the fall or after a period of hospitalization). This questionnaire comprised the following items:

- Circumstances of the injurious fall: location of the fall and the person's activity up to the moment of the fall. Participants were asked to indicate where they were at the moment they fell and if they could indicate what they were doing. Participants could choose from a list of thirteen pre-defined locations and nine pre-defined activities, or describe other locations and activities up to the moment of the fall. Two researchers (MB and JD) independently reviewed the answers to these two questions and classified the answers into two variables, fall location ( $n=10$ categories) and activity ( $n=9$ categories). Disagreements were resolved by consensus or by consulting a third party (MH).

- Causes of the fall: self-reported perceived cause of the fall. Participants were asked what, in their opinion, was the cause of their fall. They could choose from a list of thirteen predefined causes or describe other possible causes of their fall(s). More than one cause could be indicated. Two researchers ( $\mathrm{MB}$ and $\mathrm{MH}$ ) independently reviewed the answers to this question and classified the answers into two variables (intrinsic and extrinsic cause) based on two previous studies $(13,18)$. Disagreement was resolved by consensus or by consulting a third party (JD). The reported cause of a fall could be intrinsic, extrinsic, a combination of intrinsic and extrinsic, or unknown.

- Consequences of the fall: fear of falling (1 item, five-point Likert scale); activity avoidance due to fear of falling ( 1 item, five-point Likert scale), recuperation from the fall ( 1 item, fivepoint Likert scale); severity of the injury, defined as major or minor injury. Fractures, joint dislocations, and lacerations requiring sutures were considered major injuries. Lacerations without sutures, bruises, abrasions, sprains, and other minor soft tissue injuries were considered minor injuries. This classification is in accordance with the definition of major and minor injuries reported by Nevitt and colleagues (9). We asked a GP (HC) to assess all injuries that did not fit the definitions we used and to classify them into major or minor injury. 
- Socio-demographic characteristics: age; gender; living situation (living alone versus not living alone); level of education (primary school or less versus more than primary school).

- Health-related characteristics: health complaints (19 items), perceived health (first item of the RAND-36) (32), daily functioning (Frenchay Activities Index, FAI). The FAI measures participation in social and instrumental daily living activities and comprises 15 items covering three dimensions: domestic chores; work/leisure; and outdoor activities. Individual item responses capture frequency of participation ranging from 0 (never or none) to 3 (daily or weekly). Summary scores are derived by adding the items, with scores ranging from 0 (no activity) to 45 (very high participation) (33); activities of daily living disability (ADL subscale of the Groningen Activity Restriction Scale, GARS). This subscale measures disability in the domain of personal care and comprises 11 items. The items refer to what respondents are able to do and not to their actual performance. The theoretical minimum is 11 , indicating the absence of disability and the theoretical maximum 44 , indicating that a person is highly disabled(34).

\section{Statistics}

SPSS statistical software (version 13) was used for analyses. Injurious fall types were distinguished by analyzing data about fall location and activity up to the moment of the fall by means of HOMALS (homogeneity analysis by means of alternating least squares). HOMALS quantifies the nominal variables fall location (10 answer categories) and activity ( 9 answer categories) by assigning numerical values to each answer category of the two variables and to each person in the study. HOMALS identifies associations between fall location and activity in a two-dimensional plot. The outcome figure represents coordinates for every single person based on location and activity. Coordinates of persons with different answer patterns are positioned far apart, whereas persons with similar answer patterns are positioned in relatively close proximity. Persons who are located closely together in the plot constitute a homogeneous group. In this way we were able to identify injurious fall types (35).

If injurious fall types were identified we further investigated the relation between these injurious fall types on the one hand and socio-demographic characteristics, perceived cause of the fall, consequences of the fall, and health-related characteristics on the other by means of chi-square $(\alpha=0.05)$ and one-way ANOVA with Tukey's criterion for post-hoc pairwise comparisons $(\alpha=0.05)$. 


\section{RESULTS}

\section{Circumstances of the falls}

Table 1 shows the distribution of the fall locations. The majority of falls occurred outside the home. The location where most of the falls occurred was the street or sidewalk(38\%).

Table 2 shows the activities up to the moment of the fall. Not surprisingly, walking was the most prevalent activity up to the moment of a fall (21\%). A substantial proportion of the falls was mobility-related (about 45\%), while about 20\% were related to household activities.

\section{Types of injurious falls}

Figure 1 shows the distribution of persons within the two-dimensional HOMALS solution. It reduced the complexity of the available data, and yielded a two-dimensional solution with eigenvalues of 0.879 and 0.752 for the first and second dimension, respectively.

Table 1 - Distribution of fall locations $(n=333)$

\begin{tabular}{lcc}
\hline Location & Number & $(\%)$ \\
\hline Indoor locations (own home) & 36 & $(10,8)$ \\
Stairs & 31 & $(9,3)$ \\
Living room and studio at home & 18 & $(5,4)$ \\
Bedroom & 18 & $(5,4)$ \\
Hallway & 14 & $(4,2)$ \\
Bathroom & 12 & $(3,6)$ \\
Kitchen and cellar & & $(5.7)$ \\
Indoor locations (away from home) & 19 & $(10.5)$ \\
Shop, post office, church, bar, etc & & $(0,9)$ \\
Outdoor locations around one's home & 35 & \\
Access path, garden & 3 & $(100,0)$ \\
Other (balcony, terrace) & & \\
Outdoor locations away from home \\
Street or sidewalk, park, forest, pasture, playground, etc \\
Total
\end{tabular}


Table 2 - Distribution of activities up to the moment of the fall $(n=333)$

\begin{tabular}{lll}
\hline Activity & Number & $(\%)$ \\
\hline Instrumental activities of daily living (IADL) & 75 & $(22,5)$ \\
Walking & 71 & $(21,3)$ \\
Catching and moving things & 51 & $(15,3)$ \\
Activities of daily living (ADL) & 33 & $(9,9)$ \\
Lavatory visit & 22 & $(6,6)$ \\
Cycling & 19 & $(5,7)$ \\
Social activities (for example: visiting friends or family or voluntary work) & 16 & $(4,8)$ \\
Climbing stairs & 9 & $(2,7)$ \\
Other & 37 & $(11,1)$ \\
Total & 333 & $(100,0)$ \\
\hline
\end{tabular}

The first dimension represents the fall location ranging from outdoors (away from own home and around one's home) to indoors (indoor locations away from one's home and indoor in one's home (kitchen/cellar, stairs, living room/ studio at home, hallway, bedroom, bathroom). The second dimension represents the activities and ranges from lavatory visit, through outdoor activities (cycling, walking, social activities) to indoor activities (IADL, ADL, catching and moving things, and ascending and descending stairs).

We identified a group of injurious falls occurring in the bathroom/hall during lavatory visit (group 1), which is opposed to a group of outdoor falls during walking, cycling, and shopping (group 4). Furthermore, we distinguished a group of indoor falls during ADL (group 2) and a group outdoor falls around the respondents' home (garden) during IADL. This last group is located at the transition between outdoor locations and indoor locations (group 3). Based on these four groups of injurious falls, we defined the following four injurious fall types:

1. Indoor falls in the hall and bathroom, predominantly during lavatory visit

2. Indoor falls (at other locations than the hall and bathroom), predominantly during ADL

3. Outdoor falls near the home (garden, access path), predominantly during IADL

4. Outdoor falls away from home, occurring predominantly during walking, cycling, and shopping for groceries 


\section{Object Scores}

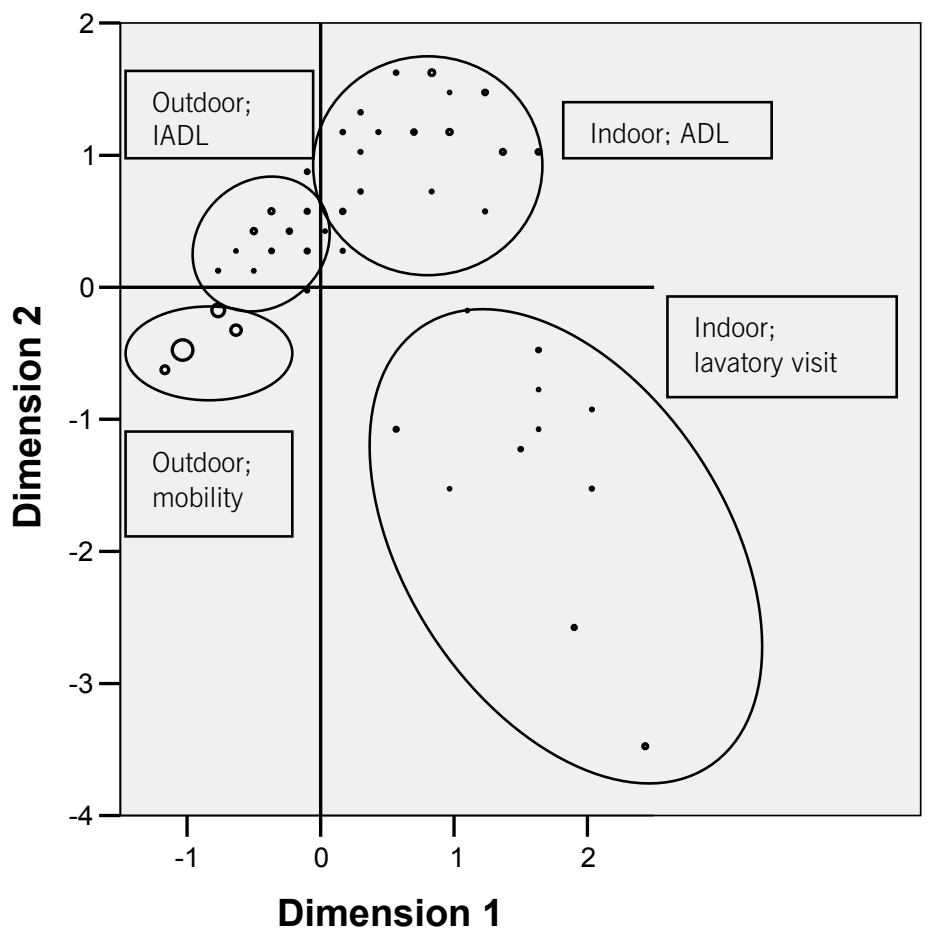

Cases weighted by number of objects.

Figure 1 shows the combined distribution of injurious falls based on the optimal quantifications for both the location of the fall and the activity up to the moment of the fall, and reveals four groups of falls. The size of the dots represents the number of subjects; the bigger a dot, the more subjects it represents.

Figure 1 - Injurious Fall Types in HOMALS Plot of Object Scores 


\section{Perceived causes and consequences of the fall}

The majority of the 333 respondents reported an extrinsic cause of their fall ( $n=169$, $51 \%$ ), whereas 112 respondents (34\%) reported an intrinsic cause of their fall. A total of 36 respondents ( $11 \%$ ) stated that the cause of their fall was a combination of intrinsic and extrinsic causes. One hundred and eighty respondents had sustained a fall resulting in a major injury (54\%). Fractures had occurred in 121 of the 333 respondents who sustained an injurious fall (36\%). About two third of the respondents experienced some fear of falling $(n=226)$, and about half $(n=183)$ avoided activities because they were afraid to fall during these activities. Recuperation after the fall was judged reasonable to good by 236 respondents $(71 \%)$.

\section{Socio-demographic characteristics}

All of the 333 participants were community-dwelling and ranged in age from 65 to 95 years, with a mean age 74.9 (SD 6.4). The majority of the study population was living with a partner at the time of the fall (77\%), had higher than primary school education (72\%), and was female (69\%).

\section{Health-related characteristics}

The 333 respondents had an average of 6 health complaints (SD 4.1) and had mean scores on the FAl and GARS of 23.5 (SD 8.7) and 17.2 (SD 6.7), respectively. A total of 302 (91\%) persons rated their health as good to excellent.

\section{Relationship between fall types and other characteristics}

Table 3 shows that intrinsic causes of falls were significantly more frequent for indoor than for outdoor locations (types 1 and 2 versus types 3 and 4). Moreover, type 4 fallers reported significantly more extrinsic causes than fallers in the other injurious fall types. We found no relationship between injurious fall type and the consequences of the fall, except for activity avoidance $(p=0.044)$. We found that persons who were younger than were predominantly involved in type 4 falls (table 4 ). Table 5 shows a number of significant differences in health-related characteristics between the four injurious fall types. We found a significant difference between type 3 and type 4 falls and between type 1 and type 4 falls in terms of the total number of health complaints. Type 4 fallers reported less health complaints. As regards the total FAl score, there was a significant 
difference between types 1 and 2 and between types 1 and 4 . Type 1 fallers had less favourable scores on the FAI. Finally, the GARS score was significantly different between type 3 and type 4 falls and between type 1 and type 4 falls. Type 4 fallers had more favourable scores on the GARS.

\section{DISCUSSION}

The circumstances under which injurious falls occur have been accurately described in previous studies $(9,25-30)$. Although fall location and activity were the most common reported circumstances in these studies, none of the studies assessed whether persons sustaining injurious falls can be classified into specific fall types based on a combination of fall location and activity up to the moment of the fall. By doing so we identified 4 injurious fall types in the present study:

1. Indoor falls in the hall and bathroom, predominantly during lavatory visits

2. Indoor falls (at other locations than the hall and bathroom), predominantly during ADL

3. Outdoor falls near the home (garden, access path), predominantly during IADL

4. Outdoor falls away from home, occurring predominantly during walking, cycling, and shopping for groceries.

We concluded that type 1 fallers (indoor fallers in the hall and bathroom during lavatory visits) proved to belong to the most inactive group (lowest FAI score), having more problems coping with activities of daily living (highest GARS score). Type 4 fallers (persons who experienced a fall away from home during mobility-related activities) predominantly were younger (aged < 80), more active and have the most favourable daily functioning (GARS) scores. This group seems to consist of those elderly people who are less frail and still venture outside. We did not find a significant difference between injurious fall types in terms of the consequences of the fall, except for activity avoidance after the fall. Indoor falls, with the exception of those in the hall and bathroom during ADL (type 2 fallers) led to fewer fractures than the other fall types (approximately 10\%). It has been suggested that indoor falls carry a lower risk of injury, because indoor surfaces may be more absorbing than outside ones (9), because persons who fall inside the house are more likely to fall on carpeted floors. Our data tend to support this suggestion. 
Table 3 - Relationship of causes and consequences of the fall with injurious fall types

\begin{tabular}{|c|c|c|c|c|c|}
\hline & Type $1^{*}$ & $\overline{T y p e ~} 2 \dagger$ & Type 3‡ & Type 4§ & \\
\hline Distribution of participants & $32(9.6)$ & $116(34.8)$ & $38(11.4)$ & $147(44.1)$ & \\
\hline Causes of the fall & & & & & 0.000 \\
\hline Intrinsic cause & $21(18.8)$ & $49(43.8)$ & $13(11.6)$ & $29(25.9)$ & \\
\hline Extrinsic cause & $3(1.8)$ & $50(29.6)$ & $20(11.8)$ & $96(56.8)$ & \\
\hline \multicolumn{6}{|l|}{ Consequences } \\
\hline Injury & & & & & 0.622 \\
\hline$\%$ Major injury & $16(8.9)$ & $58(32.2)$ & $22(12.2)$ & $84(46.7)$ & \\
\hline \% Minor injury & $16(10.5)$ & $58(41.2)$ & $16(10.5)$ & $63(41.2)$ & \\
\hline Injury & & & & & 0.172 \\
\hline$\%$ Fracture & $12(9.9)$ & $33(27.3)$ & 15 (12.4) & $61(50.4)$ & \\
\hline$\%$ No fracture & $20(9.4)$ & $83(39.2)$ & $23(10.8)$ & $86(40.6)$ & \\
\hline Recuperation from the fall & & & & & 0.755 \\
\hline$\% \geq$ reasonable & $21(8.9)$ & $83(35.2)$ & $25(10.6)$ & $107(45.3)$ & \\
\hline$\% \leq$ moderate & $11(11.3)$ & $33(34.0)$ & $13(13.4)$ & $40(41.2)$ & \\
\hline
\end{tabular}

*Type 1: Indoor falls in the hall and bathroom, during lavatory visit

†Type 2: Indoor falls (at other locations than the hall and bathroom), during ADL

‡Type 3: Outdoor falls near the home, predominantly during IADL

§Type 4: Outdoor falls away from home, occurring during mobility-related activities

Row totals add up to $100 \%$ for each of the categories listed 


\section{Table 4 - Relationship of socio-demographic characteristics and health-related Characteristics with injurious fall types}

\begin{tabular}{|c|c|c|c|c|c|}
\hline & Type $1 *$ & Type $2 \dagger$ & Type $3 \ddagger$ & Type $4 \S$ & P-value \\
\hline $\begin{array}{l}\text { Distribution of participants within } \\
\text { fall types }\end{array}$ & $32(9.6)$ & $116(34.8)$ & $38(11.4)$ & $147(44.1)$ & \\
\hline \multicolumn{6}{|l|}{ Socio-demographic characteristics } \\
\hline Age & & & & & 0.036 \\
\hline$\%<80$ vear & $22(8.6)$ & $82(32.0)$ & $28(10.9)$ & $124(48.4)$ & \\
\hline$\% \geq 80$ vear & $10(13.0)$ & $34(44.2)$ & $10(13.0)$ & $23(29.9)$ & \\
\hline Gender & & & & & 0.121 \\
\hline$\%$ Female & $21(9.2)$ & $(35.1)$ & $(8.8)$ & $(46.9)$ & \\
\hline$\%$ Male & $11(10.5)$ & $36(34.3)$ & $18(17.1)$ & $40(38.1)$ & \\
\hline Living situation & & & & & 0.850 \\
\hline \% Living alone & $14(9.7)$ & 48 (33.3) & $15(10.4)$ & $67(46.5)$ & \\
\hline \% Living with a partner & $18(9.6)$ & $68(36.2)$ & $23(12.2)$ & $79(42.0)$ & \\
\hline Level of education & & & & & 0.748 \\
\hline$\% \leq$ primarv school & $10(10.6)$ & $33(35.1)$ & $13(13.8)$ & $38(40.4)$ & \\
\hline$\%>$ primarv school & $22(9.2)$ & $83(34.7)$ & $25(10.5)$ & $109(45.6)$ & \\
\hline \multicolumn{6}{|l|}{ Health-related characteristics } \\
\hline Fear of falling & & & & & 0.981 \\
\hline$\% \geq$ sometimes & $22(9.7)$ & $80(35.4)$ & $26(11.5)$ & $98(43.4)$ & \\
\hline$\% \leq$ almost never & $10(9.3)$ & $36(33.6)$ & $12(11.2)$ & $49(45.8)$ & \\
\hline Activitv avoidance & & & & & 0.044 \\
\hline$\% \geq$ sometimes & $20(10.9)$ & $71(38.8)$ & $24(13.1)$ & $68(37.2)$ & \\
\hline$\% \leq$ almost never & $12(8.0)$ & $45(30.0)$ & $14(9.3)$ & $79(52.7)$ & \\
\hline Perceived health ( $\geq$ good) & & & & & 0.546 \\
\hline$\% \geq$ good & $31(10.3)$ & $105(34.8)$ & $33(10.9)$ & $133(44.0)$ & \\
\hline$\% \leq$ moderate & $1(3.2)$ & $11(35.5)$ & $5(16.1)$ & $14(45.2)$ & \\
\hline
\end{tabular}

*Type 1: Indoor falls in the hall and bathroom, during lavatory visit

†Type 2: Indoor falls (at other locations than the hall and bathroom), during ADL

$\ddagger$ Type 3: Outdoor falls near the home, predominantly during IADL

§Type 4: Outdoor falls away from home, occurring during mobility-related activities

Row totals add up to $100 \%$ for each of the categories listed 
Table 5 - ANOVA of health-related characteristics and injurious fall types

\begin{tabular}{|c|c|c|c|c|c|c|}
\hline & $\begin{array}{l}\text { Type } 1^{*} \\
(n=32)\end{array}$ & $\begin{array}{l}\text { Type } 2 \dagger \\
(n=116)\end{array}$ & $\begin{array}{l}\text { Type 3‡ } \\
(n=38)\end{array}$ & $\begin{array}{l}\text { Type 4\$ } \\
(n=147)\end{array}$ & $\begin{array}{l}\text { P-value } \\
\text { (ANOVA) }\end{array}$ & P-value \\
\hline Total health & 7.75 & 6.34 & 8.26 & 5.29 & 0.000 & 0.010 (types 1 and 4 ) \\
\hline complaints & & & & & & 0.000 (types 3 and 4) \\
\hline Total FAI|| score & 18.94 & 23.32 & 21.61 & 25.03 & 0.001 & 0.050 (types 1 and 2) \\
\hline$(0-45) \#$ & & & & & & 0.002 (types 1 and 4 ) \\
\hline Total GARS I score & 20.16 & 17.36 & 19.58 & 15.90 & 0.001 & 0.010 (types 1 and 4) \\
\hline$(11-44) \#$ & & & & & & 0.000 (types 3 and 4 ) \\
\hline
\end{tabular}

*Type 1: Indoor falls in the hall and bathroom, during lavatory visit

†Type 2: Indoor falls (at other locations than the hall and bathroom), during ADL

‡Type 3: Outdoor falls near the home, predominantly during IADL

§Type 4: Outdoor falls away from home, occurring during mobility-related activities

|| Frenchay Activities Index; đGroningen Activity Restriction Scale; \#the underlined score is the most favourable score

Our finding that a majority of the injurious falls took place outdoors is consistent with previous reports (5, 27-29). Walking accounted for the largest proportion of the activities respondents were engaged in, as was also reported from previous studies $(5,28,29,36)$. The younger age group was more often engaged in leisure activities and sustained more outdoor falls. The more frail older persons in our study tended to stay in their own house and predominantly fell during ADL and particularly during lavatory visits. These findings resemble the findings of previous studies, which found that vigorous persons were more likely to fall outside the home during displacement activities such as climbing ladders or engaging in sports, while frail older persons fell during routine daily activities at home (37-39). 
The present study has some limitations. First, all subjects in our sample sustained an injurious fall and attended the A\&E department of a hospital to get treatment for the consequences of their injurious falls. We did not include persons who visited their GP with the consequences of an injurious fall. Moreover, we also did not select those persons who did not seek medical attention at all for the consequences of the injurious fall. So, we included a group with serious injuries after a fall. Second, all data were self-reported. Although the accuracy of selfreport data remains unclear, older people are often the only witnesses of their fall events, so self-reports remain an important source of information about falls (40). Third, it should be noted that that the analyses are data-driven, meaning that there was no a priori hypotheses formulated. HOMALS was allowed to come up with the best partitioning between the four fall types.

In conclusion, we succeeded in classifying injurious falls based on fall location and activity up to the moment of the fall. The face validity of the injurious fall typology is obvious. We did not find any relationship between the four injurious fall types and severity of the consequences of the fall. However, there seems to be a difference in fall location and activity up to the moment of the fall between the younger and more active elderly, who still go outdoors, and the more frail older people who tend to stay indoors. Those persons who fell outdoors predominantly reported an extrinsic cause of their fall, whereas those persons who fell indoors reported an intrinsic cause. Our data suggests that in case of a faller ( $<80$ year) who has fallen outside and a faller ( $\geq 80$ year) who has fallen inside we should have special attention for extrinsic causes and intrinsic causes, respectively. However, the conclusions reached in this exploratory analysis are tentative and need to be validated in a separate dataset.

\section{ACKNOWLEDGEMENTS AND FUNDING}

We would like to thank Natasja van Veen for her contribution to the data collection and the original idea for this manuscript. This study was funded by the Netherlands Organization for Health Research and Development (ZonMw), grant number 945-02-053. 


\section{REFERENCES}

1. Gillespie LD, Gillespie WJ, Robertson MC, LambSE, Cumming RG, Rowe BH. Interventions for preventing falls in elderly people. Cochrane Database Syst Rev. 2003(4):CD000340.

2. Chang JT, Morton SC, Rubenstein LZ, Mojica WA, Maglione M, Suttorp MJ, et al. Interventions for the prevention of falls in older adults: systematic review and meta-analysis of randomised clinical trials. Bmj. 2004;328(7441):680.

3. Kannus $P$, Sievanen $H$, Palvanen $M$, Jarvinen $T$, Parkkari J. Prevention of falls and consequent injuries in elderly people. Lancet. 2005 Nov 26;366(9500):1885-93.

4. Resnick B. Falls in a community of older adults: putting research into practice. Clin Nurs Res. 1999 Aug;8(3):251-66.

5. Yasamura S, Haga H, Niino N. Circumstances of injurious falls leading to medical care among elderly people living in a rural community. Archives of Gerontology and Geriatrics. 1996;23:95-109.

6. King MB, Tinetti ME. Falls in community-dwelling older persons. J Am Geriatr Soc. 1995 Oct;43(10):1146-54.

7. Kannus P, Parkkari J, Koskinen S, Niemi S, Palvanen M, Jarvinen M, et al. Fall-induced injuries and deaths among older adults. JAMA. 1999 May 26;281(20):1895-9.

8. Luukinen H, Koski K, Honkanen R, Kivela SL. Incidence of injury-causing falls among older adults by place of residence: a population-based study. J Am Geriatr Soc. 1995 Aug;43(8):871-6.

9. Nevitt MC, Cummings SR, Hudes ES. Risk factors for injurious falls: a prospective study. J Gerontol. 1991;46(5):M164-70.

10. O'Loughlin J, Robitaille Y, Bolvin J-F, Suissa S. Incidence of and risk factors for falls and injurious falls among the community-dwelling elderly. Am J Epidemiol. 1993;137:342-54.

11. Tinetti ME, Speechley M, Ginter SF. Risk factors for falls among elderly persons living in the community. N Eng/ J Med. 1988;319(26):1701-7.

12. Hoidrup S, Sorensen TI, Gronbaek M, Schroll M. Incidence and characteristics of falls leading to hospital treatment: a one-year population surveillance study of the Danish population aged 45 years and over. Scand J Public Health. 2003;31(1):24-30.

13. Bloem BR, Boers, I., Cramer, M., Westendorp, R.G.J., Gerschlager, W. Falls in the elderly: identification of risk factors. Wiener Klinische Wochenschrift. 2001;113:352-62.

14. Vellas BJ, Wayne SJ, Romero LJ, Baumgartner RN, Garry PJ. Fear of falling and restriction of mobility in elderly fallers. Age Ageing. 1997;26:189-93.

15. Scuffham P, Chaplin S, Legood R. Incidence and costs of unintentional falls in older people in the United Kingdom. J Epidemiol Community Health. 2003 Sep; 57(9):740-4. 
16. Newton JL, Kyle P, Liversidge P, Robinson G, Wilton $K$, Reeve $P$. The costs of falls in the community to the North East Ambulance Service. Emerg Med J. 2006 Jun;23(6):479-81.

17. Meerding WJ, Mulder S, van Beeck EF. Incidence and costs of injuries in The Netherlands. Eur J Public Health. 2006 Jun; 16(3):272-8.

18. Bueno Cavanillas A, Padilla Ruiz F, Jimenez Moleon JJ, Peinado Alonso CA, Galvez Vargas R. Risk factors in falls among the elderly according to extrinsic and intrinsic precipitating causes. Eur J Epidemiol. 2000;16(9):849-59.

19. Carroll NV, Slattum PW, Cox FM. The cost of falls among the community-dwelling elderly. J Manag Care Pharm. 2005 May; 11(4):307-16.

20. Hendriks MR, Evers SM, Bleijlevens MH, van Haastregt JC, Crebolder HF, van Eijk JT. Cost-effectiveness of a multidisciplinary fall prevention program in community-dwelling elderly people: A randomized controlled trial (ISRCTN 64716113). Int J Technol Assess Health Care. 2008 Spring;24(2):193-202.

21. Tinetti ME. Multifactorial fall-prevention strategies: time to retreat or advance. J Am Geriatr Soc. 2008 Aug;56(8):1563-5.

22. McClure R, Turner C, Peel N, Spinks A, Eakin E, Hughes K. Population-based interventions for the prevention of fall-related injuries in older people. Cochrane Database Syst Rev. 2005(1):CD004441.

23. Gates S, Lamb SE, Fisher JD, Cooke MW, Carter YH. Multifactorial assessment and targeted intervention for preventing falls and injuries among older people in community and emergency care settings: systematic review and meta-analysis. Bmj. 2008 Jan 19;336(7636):130-3.

24. Beswick AD, Rees K, Dieppe P, Ayis S, Gooberman-Hill R, Horwood J, et al. Complex interventions to improve physical function and maintain independent living in elderly people: a systematic review and meta-analysis. Lancet. 2008 Mar 1;371(9614):725-35.

25. Nachreiner NM, Findorff MJ, Wyman JF, McCarthy TC. Circumstances and consequences of falls in community-dwelling older women. J Womens Health (Larchmt). 2007 Dec;16(10):1437-46.

26. Gazzola JM, Gananca FF, Aratani MC, Perracini MR, Gananca MM. Circumstances and consequences of falls in elderly people with vestibular disorder. Rev Bras Otorrinolaringol (Eng/ Ed). 2006 MayJun; 72(3):388-92.

27. Mackenzie L, Byles J, Higginbotham N. A prospective community-based study of falls among older people in Australia: frequency, circumstances, and consequences. OTJR. 2002;22(4):143-52.

28. Hill K, Schwarz J, Flicker L, Carroll S. Falls among healthy, community-dwelling, older women: a prospective study of frequency, circumstances, consequences, and prediction accuracy. Australian and New Zealand Journal of Public Health. 1999;23:41-8. 
29. Berg WP, Alessio HM, Mills EM, Tong C. Circumstances and consequences of falls in independent community-dwelling older adults. Age Ageing. 1997;26(4):261-8.

30. Campbell AJ, Borrie MJ, Spears GF, Jackson SL, Brown JS, Fitzgerald JL. Circumstances and consequences of falls experienced by a community population 70 years and over during a prospective study. Age Ageing. 1990;19(2):136-41.

31. Hendriks MR, van Haastregt JC, Diederiks JP, Evers SM, Crebolder HF, van Eijk JT. Effectiveness and costeffectiveness of a multidisciplinary intervention programme to prevent new falls and functional decline among elderly persons at risk: design of a replicated randomised controlled trial [ISRCTN64716113]. BMC Public Health. 2005 Jan 14;5:6.

32. van der Zee KI, Sanderman R. Het meten van de algemene gezondheidstoestand met de RAND-36: een handleiding [Measuring general health with the RAND-36:manual]. Groningen: Noordelijk Centrum voor Gezondheidsvraagstukken, Rijksuniversiteit Groningen; 1993.

33. Schuling J, de Haan R, Limburg M, Groenier KH. The Frenchay Activities Index. Assessment of functional status in stroke patients. Stroke. 1993 Aug;24(8):1173-7.

34. Kempen GI, Miedema I, Ormel J, Molenaar W. The assessment of disability with the Groningen Activity Restriction Scale. Conceptual framework and psychometric properties. Soc Sci Med. 1996 Dec;43(11):1601-10.

35. Baumert U, Golan I, Redlich M, Aknin JJ, Muessig D. Cleidocranial dysplasia: molecular genetic analysis and phenotypic-based description of a Middle European patient group. Am J Med Genet A. 2005 Dec 1;139(2):78-85.

36. Ryynanen OP, Kivela SL, Honkanen R. Times, places, and mechanisms of falls among the elderly. Zeitschrift fur Gerontologie. 1991;24:154-61.

37. Speechley M, Tinetti M. Falls and injuries in frail and vigorous community elderly persons. J Am Geriatr Soc. 1991;39(1):46-52.

38. Talbot LA, Musiol RJ, Witham EK, Metter EJ. Falls in young, middle-aged and older community dwelling adults: perceived cause, environmental factors and injury. BMC Public Health. 2005;5:86.

39. Stalenhoef PA, Diederiks JP, Knottnerus JA, de Witte LP, Crebolder HF. The construction of a patient record-based risk model for recurrent falls among elderly people living in the community. Fam Pract. 2000;17(6):490-6.

40. Dickens J, Jones M, Johansen A. Falls definition--reliability of patients' own reports. Age Ageing. 2006 Jul;35(4):450-1. 


\section{WILL AN INJURIOUS FALLER}

FALL AGAIN?

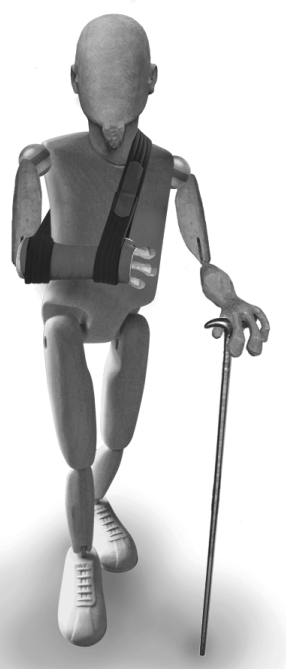

Michel HC Bleijlevens

Marike RC Hendriks Jolanda CM van Haastregt Harry FJM Crebolder Jacques ThM van Eijk 


\section{ABSTRACT}

\section{Objective}

We assessed which risk factors predict new falls among a group of community-dwelling injurious fallers. Furthermore, we aimed to develop a feasible fall risk screening tool in order to select those patients who are at most risk sustaining a new fall.

\section{Study design and Setting}

We explored which risk factors are associated with a new fall among community-dwelling elderly people aged $\geq 65$ years who attended the Accident \& Emergency department at a University Hospital in the Netherlands $(n=333)$.

\section{Results}

A total of 15 variables were associated with a new fall $(p<0.05)$, of which recurrent falls in previous years, consequences of previous falls, ADL dependency, mobility and age were most strongly associated. A multivariable backward logistic regression model resulted in a fall risk screening tool comprising: age ( $\geq 79$ year), recurrent falls in the previous year, experiencing more handicaps associated with previous fall(s), and having joint disorders. The total risk score of the fall risk screening tool ranges from 0 to 11 , which corresponds to a probability ranging from $15 \%$ to $90 \%$.

\section{Conclusion}

The results indicate that the developed fall risk screening tool can improve the efficiency to select people at high-risk for sustaining a new fall in the current study population. 


\section{INTRODUCTION}

Falls and their consequences are increasingly recognised as a serious threat to the health and independency of older people (1-3). Approximately one third of all community-dwelling people aged 65 years or over sustain at least one fall each year (2-7). As a result of the ageing population, the societal burden and costs related with falls will increase further unless we are able to take effective preventive measures.

It is supposed that reducing risk factors for falls prevents future fall incidents. In general, these risk factors can be classified as intrinsic and extrinsic risk factors. Intrinsic risk factors typically include factors such as balance and gait disorders, and cognitive impairment. Extrinsic risk factors are hazards in the living environment, such as slippery floors or loose carpets (8-10). A fall can rarely be contributed to a single intrinsic or extrinsic cause (1, 1116). In general a fall is the result of the interaction between several intrinsic and extrinsic risk factors (16). Therefore, fall prevention programmes should be aimed at reducing both intrinsic and extrinsic risk factors (5-7).

Multifactorial fall prevention programmes addressing a wide range of risk factors are considered to be the most promising strategy to prevent falls, especially if they are aimed at people at high-risk $(5-7,17,18)$. However, a recent systematic review concluded that the effectiveness of these multifactorial programmes is less convincing than previously assumed (3). When searching for possibilities to optimise the effectiveness of multifactorial programmes we may consider optimizing at least two aspects. First, the way these multifactorial programmes are delivered, and second the way the high-risk target populations are selected. The current paper focuses on the second aspect, the selection of persons at high-risk for falling. Fall prevention programmes often select high-risk populations based on risk factors such as having a history of recurrent or injurious falls, or having mobility impairments (19). However, there are indications that this is a rather inefficient procedure, because even when one aims to select high-risk persons based on these criteria (19), often a substantial number of relatively low risk persons are also included. Therefore it may be doubted whether the current criteria for high-risk status are sufficient. In order to increase the efficiency of multifactorial fall prevention programmes it is of utmost importance to develop more efficient methods to select older people at high risk for falls. This may substantially increase the effectiveness and efficiency of these programmes. 
We recently evaluated the effectiveness of a multifactorial fall prevention programme among community-dwelling people aged 65 years or over who have visited the A\&E department of a hospital with the consequences of a fall. The programme consists of a detailed medical and occupational-therapy assessment resulting in recommendation and/or referral to relevant services if indicated (20). Results of this randomised controlled trial showed no effects of this programme on the incidence of falls or daily functioning (21). A process evaluation revealed that the practitioners who performed the fall prevention programme experienced that the group of selected 'high-risk' persons with a history of an injurious fall a also comprises a substantial number of persons without a high fall risk (22). It is therefore important to explore whether an additional screening for increased fall risk within a (potentially) high-risk group of injurious fallers, can further improve the selection of high-risk persons, which may increase the efficiency of multifactorial fall prevention programmes. The primary aim of this paper is therefore to asses which risk factors predict new falls among a group of communitydwelling injurious fallers aged 65 years of over. Furthermore, dependent of the results we aim to develop a feasible fall risk screening tool for the involved healthcare professionals, like professionals working on the A\&E department of a hospital and general practitioners (GP's). In this way we hope to tailor the selection procedure more to patients who are at most in need for preventive action.

\section{METHODS}

\section{Design}

This study is a secondary analysis of data obtained from the trial mentioned above $(20,21)$. An explorative approach has been used to examine which variables predict new falls during the follow up period among this group of older people. Subsequently, a fall risk screening tool was developed in order to identify persons who actually are at high-risk for sustaining a new fall.

\section{Study population}

The study population was derived from the previously mentioned two-group randomised controlled trial assessing the effectiveness and cost-effectiveness of a multidisciplinary fall prevention programme among community-dwelling people, aged 65 years or over who 
attended the A\&E department of a hospital after an injurious fall $(20,21)$. Participants randomly allocated to the intervention group received a medical and occupational-therapy assessment followed by recommendations and/or further referral if indicated, while participants allocated to the control group received usual care. The trial had a follow-up period of 12 months $(20,21)$. A total of 333 community-dwelling elderly people aged 65 years and over living in Maastricht (the Netherlands) or its surrounding area were included in the RCT. As we did not find any effect of the multifactorial fall prevention programme on the incidence of falls or daily functioning, data of all 333 participants of the RCT were available for the current study.

\section{Measurements}

The participants received a baseline questionnaire which assessed the following variables:

- Socio-demographic characteristics: age; gender; living situation (living alone versus not living alone); and level of education (primary school or less versus more than primary school).

- Fall History: Recurrent falls in the previous year (yes/no).

- Handicaps associated with previous fall(s): Falls Handicap Inventory (FHI, 18 items, total score ranging from 0 to 72 where 0 is the most favourable score) (23).

- Health-related characteristics: psychoactive drug use (yes/no); number of self-reported diseases (20 items); number of self-reported health complaints (19 items). Both information on the total number of diseases $(n=20)$ and health complaints $(n=19)$ as well as the presence of 4 diseases and 7 health complaints individually was gathered (yes/no). These 4 diseases (lung disease, joint disorder, balance disorder, and history of stroke) and 7 health complaints (Dizziness, pain, foot problems, problems with keeping balance, consequences of stroke, poor vision, and poor hearing were taken into account individually because the were found to be a risk factor for falling in literature(24)

- Activity and mobility: daily functioning as measured with the Frenchay Activities Index (FAl, 15 items, total score ranging from 0 to 45 where 45 is the most favourable score) (25); activities of daily living (ADL) and instrumental ADL disability as measured with the Groningen Activity Restriction Scale (GARS, 11 items, total score ranging from 11 to 44 where 11 is the most favourable score) (26); mobility as measured with the first item of the European Quality of Life instrument (EuroQol, 1 item, three-point Likert scale, ranging from 1 (no problems walking about) to 3 (confined to bed)) $(27,28)$. 
- Psychosocial characteristics: fear of falling (1 item, five-point Likert scale ranging from 1 (never) to 5 (very often)); activity avoidance due to fear of falling ( 1 item, five-point Likert scale ranging from 1 (never) to 5 (very often)); and depression as measured with the Hospital Anxiety and Depression Scale (subscale depression HADS , 7 items, total score ranging from 0 to 21 where 0 is the most favourable score), using the recommended cut-off point of 11 (definite cases vs. doubtful/no cases) (29, 30);

- Life style characteristics: Alcohol consumption (Heavy alcohol consumption was defined as drinking 18 glasses or more per week);

- Other fall related variables: Presence of dogs or cats in the household (yes/no).

Furthermore, participants recorded their falls continuously on a fall calendar during twelve months after completing the baseline questionnaire. They were contacted once every month by telephone by an independent call centre to report the falls they noted on the calendar. A fall was defined as an event in which a person is coming to rest unintentionally on the ground or other lower level (31). Recurrent fallers were defined as fallers with two or more falls in the previous year.

\section{Data analysis}

First, the two categorical variables (fear of falling and activity avoidance) and six continuous variables (age, handicaps associated with previous fall(s), number of self-reported diseases, number of self-reported health complaints, daily functioning, $A D L$ and instrumental ADL disability, and depression were dichotomised (yes/no). For the HADS we used the recommended cut-off value of 11 (30). The cut-off values for the other 6 continues variables were determined based on sensitivity and specificity. Sensitivity and specificity were assessed at different cut-off values for each of the variables in order to determine the maximum sum of sensitivity and specificity as the statistical optimal cut-off value. Second, associations between sustaining a fall within one year follow up, as dependent variable and some of the possible predictors as independent variable were assessed by means of univariate logistic regression (Wald-test). Third, in order to come to the most appropriate model to predict a new fall, all possible predictors showing a significant association with a new fall $(p<0.05$ on the Waldtest), were entered simultaneously in a multivariable backward logistic regression model. Fourth, variables were deleted from the initial model on the basis of lack of significance on the Wald-test (threshold for significance was $p<0.05$ ), resulting in a model comprising the 
strongest predictors of a new fall. The goodness-of-fit of this model was tested using the Hosmer-Lemeshow test (32). Fifth, the probability of a new fall for all possible combinations of outcomes from the model were calculated (predicted probabilities). Sixth, to facilitate the use of the model in clinical practice, we transformed the regression coefficients of the identified predictors of a new fall in the model (multiplied with a factor 3 , rounded off to the nearest integer) into aggregated total risk scores which can be obtained easily by adding up the scores of each of the selected predictors. Finally, a receiver operating characteristic (ROC) curve was plotted to estimate the discriminating power of the model. This is a plot of the sensitivity against 1 -specificity at various cut-off points of the total risk score, indicating at what level of the score patients will be classified as fallers or non fallers. The area under the ROC curve (AUC) is an estimate of how well persons who fell will be discriminated from persons who did not fall. An AUC of 1 represents a perfect discrimination, whereas an AUC of 0.5 refers to a model with no discrimination at all (33). In addition, positive and negative predictive values (PV+ and PV-) were calculated.

\section{RESULTS}

\section{Participants}

Of the 333 persons included in the main study (RCT), 190 (57\%) completed the total follow up period. Therefore these will be used for the present analyses. For these 190 persons we have the complete fall history over the one year fall follow-up period available. The mean age of these 190 participants was $74.5 \pm 6.2$ years. The majority was female $(66.8 \%)$, lived together with someone (56.8\%) and had more then primary school education (75.3\%).

We compared the possible risk factors measured at baseline between the 190 persons who were enrolled in the current analyses and the 143 participants who were not enrolled. The latter group was more dependent (lower scores on the GARS at baseline, 16.6 versus 18.1, $\mathrm{p}=0.047$ ), reported more handicaps associated with previous fall(s) (higher scores on the $\mathrm{FHI}, 26.2$ versus 19.9, $\mathrm{p}=0.004$ ), reported more health complaints (6.8 versus $5.8, \mathrm{p}=$ $0.034)$, had more problems with walking (62.9\% versus $48.9 \%, p=0.011$ ), and avoided activities more because they experienced fear of falling when doing those activities $(63.6 \%$ versus $48.4 \%, p=0.006$ ). 


\section{Falls}

Within the one year follow-up, 86 of the 190 participants reported 267 falls. A total of 39 participants (20.5\%) reported one fall and 47 participants $(24.7 \%)$ reported two or more falls.

\section{Cut-off points}

Table 1 shows sensitivity and specificity of the optimal cut-off points for the categorical and continuous variables for which no cut-off points were reported in literature.

Table 1 - Sensitivity and specificity of the optimal cut-off points for the dichotomised categorical and continuous variables for which no cut-off point was reported in literature

\begin{tabular}{|c|c|c|c|}
\hline Jptimal cut-off point & SE & $\mathrm{SP}$ & $\bar{\Sigma}$ \\
\hline Age (65-78 vs. $\geq 79$ ) & 38.4 & 79.8 & 118.2 \\
\hline Jandicaps associated with previous falls ( $0-23$ vs. $\geq 24$ ) & 52.4 & 74.0 & 126.4 \\
\hline Vumber of self-reported diseases ((0-1 vs. $\geq 2)$ & 75.6 & 36.5 & 112.1 \\
\hline Vumber of self-reported health complaints ( $0-6$ vs. $\geq 7$ ) & 48.8 & 70.2 & 119.0 \\
\hline Jaily functioning (0-17 vs. $\geq 18$ ) & 30.2 & 87.5 & 117.7 \\
\hline$A D L$ and instrumental ADL disability (11-14 vs. $\geq 15$ ) & 62.8 & 65.4 & 128.2 \\
\hline =ear of falling (never vs. $\geq$ almost never) & 88.4 & 27.9 & 116.3 \\
\hline Activity avoidance (never, almost never vs. $\geq$ sometimes) & 59.3 & 60.6 & 119.9 \\
\hline
\end{tabular}

$\mathrm{SE}=$ sensitivity; $\mathrm{SP}=$ specificity; $\Sigma=$ sum of sensitivity and specificity

\section{Predictors of a new fall}

Table 2 presents the results of univariate logistic regression analyses. A total of 15 variables were associated with a new fall $(p<0.05)$ and were subsequently entered into a multivariable logistic regression model. In our population recurrent falls in previous years, consequences of previous falls, $A D L$ dependency, mobility and age are most strongly associated with a new fall. 


\section{Table 2 - Univariate odds ratios and 95\% confidence intervals for possible predictors} of a new fall $(n=190)$.

Possible predictor

Cut-off point for

$\mathrm{OR}(95 \% \mathrm{Cl})$

P-value

increased fall risk

\begin{tabular}{|c|c|c|c|}
\hline Age (<79 vs. $\geq 79)$ & $\geq 79$ & $2.46(1.29-4.70)$ & 0.006 \\
\hline Gender (women vs. men) & women & $1.55(0.84-2.87)$ & 0.163 \\
\hline Living situation (alone vs. not alone) & living alone & $1.53(0.86-2.72)$ & 0.151 \\
\hline Level of education & $\leq$ primary school & $0.77(0.39-1.50)$ & 0.443 \\
\hline Recurrent falls in previous year (no versus yes) & yes & $5.15(2.77-9.56)$ & 0.000 \\
\hline Handicaps associated with previous fall(s) & $\geq 24$ & $3.13(1.70-5.76)$ & 0.000 \\
\hline Lung disease (no vs. yes) & yes & $0.96(0.42-2.18)$ & 0.926 \\
\hline Joint disorder (no vs. yes) & yes & $2.15(1.17-3.92)$ & 0.013 \\
\hline Balance disorder (no vs. yes) & yes & $1.71(0.88-3.33)$ & 0.112 \\
\hline History of stroke (no vs. yes) & yes & $2.91(1.06-8.02)$ & 0.039 \\
\hline Dizziness (no vs. yes) & yes & $1.69(0.92-3.12)$ & 0.094 \\
\hline Pain (no vs. yes) & yes & $1.11(0.60-2.05)$ & 0.741 \\
\hline Foot problems (no vs. yes) & yes & $2.25(1.16-4.35)$ & 0.016 \\
\hline Problems with keeping balance (no vs. yes) & yes & $2.07(1.11-3.86)$ & 0.023 \\
\hline Consequences of stroke (no vs. yes) & yes & $4.05(1.26-13.07)$ & 0.019 \\
\hline Poor vision (no vs. yes) & yes & $1.80(0.94-3.45)$ & 0.077 \\
\hline Poor hearing (no vs. yes) & yes & $1.41(0.77-2.56)$ & 0.263 \\
\hline Number of self-reported diseases ((0-1 vs. $\geq 2$ ) & $\geq 2$ & $1.78(0.95-3.36)$ & 0.074 \\
\hline Number of self-reported health complaints & $\geq 7$ & $2.25(1.24-4.08)$ & 0.008 \\
\hline Psychoactive drug use (no vs. yes) & yes & $0.70(0.37-1.34)$ & 0.283 \\
\hline Daily functioning & $\geq 18$ & $3.03(1.45-6.37)$ & 0.003 \\
\hline Activities of daily living(11-14 vs. $\geq 15)$ & $\geq 15$ & $3.19(1.76-5.78)$ & 0.000 \\
\hline Mobility (no problems walking about vs. $\geq$ some & $\geq$ problems & $2.35(1.31-4.22)$ & 0.004 \\
\hline Fear of falling (never vs. $\geq$ almost never) & $\geq$ almost never & $2.94(1.34-6.45)$ & 0.007 \\
\hline Activity avoidance (never vs. $\geq$ almost never) & $\geq$ sometimes & $2.24(1.25-4.01)$ & 0.007 \\
\hline Depression & definite cases & $2.69(1.04-7.02)$ & 0.042 \\
\hline Alcohol consumption & $\geq 18$ glasses per & $0.23(0.03-2.03)$ & 0.187 \\
\hline Dogs or cats in household (no vs. yes) & yes & $0.62(0.28-1.37)$ & 0.235 \\
\hline
\end{tabular}

$\mathrm{OR}=$ odds ratio 


\section{Risk of a new fall}

All possible predictors showing an association of $p<0.05$ were entered simultaneously in a multivariable backward logistic regression model, resulting in a risk model comprising four predictors: age ( $\geq 79$ year), two or more falls in the previous year, experiencing more handicaps associated with previous fall(s) ( $F H I \geq 24$ ), and having joint disorders (see table 3 ). The Hosmer-Lemeshow goodness-of-fit test for the multivariable backward logistic regression was not significant $(p=0.72)$, indicating that the risk model fits the data quite well.

To facilitate the interpretation of a person's risk of a new fall, the regression coefficients of the identified predictors of a new fall in the model (table 3 ) are multiplied with a factor 3 and rounded off to the nearest integer. In this way we obtained simple scores that can be added up into a total risk score (table 4). In addition, table 4 shows that the total risk score of this tool ranges from 0 , when all predictors are absent, to 11 , when all predictors are present. The total risk scores and the predicted probabilities for a new fall for all 16 possible combinations derived from the 4 variables in the risk model are also presented in table 4 . The probability of a new fall ranged from $15 \%$ when all predictors were absent to $90 \%$ when they all were present. In addition, table 5 demonstrates that the statistically optimal cut-off point of the fall risk screening tool is reached at a total risk score of 5 . At this score, almost half of the participants will be classified into the high-risk group (49\%) and $67.4 \%$ of the participants with a score of 5 or higher are correctly diagnosed as a future faller $(P V+)$, whereas $73.1 \%$ of the participants with a score below 4 were correctly diagnosed as no fallers (PV-). The risk of a new fall in the entire study population is $45.3 \%$ (prior probability).

\section{Table 3 - Risk model for the prediction of new falls}

\begin{tabular}{lcc}
\hline Predictor & Regression coefficient (B) & OR (95\% Cl) \\
\hline Constant & -1.75 & \\
Age $(\geq 79)$ & 0.87 & $2.40(1.16-4.95)$ \\
Recurrent falls in previous year & 1.48 & $4.39(2.28-8.45)$ \\
Handicaps associated with previous falls $(>24)$ & 0.79 & $2.21(1.13-4.43)$ \\
Having joint disorders & 0.76 & $2.13(1.08-4.20)$ \\
\hline
\end{tabular}

$\mathrm{OR}=$ odds ratio; $\mathrm{Cl}=$ confidence interval 


\section{Table 4 - Fall risk screening tool for the prediction of a new fall}

\begin{tabular}{|c|c|c|}
\hline Predictors & Total risk score & $\mathrm{PP}(\%)$ \\
\hline None & 0 & 15 \\
\hline A (Having joint disorders) & 2 & 27 \\
\hline B (Experiencing more handicaps associated with previous fall(s) (FHI $\geq 24)$ ) & 2 & 28 \\
\hline C (Age ( $\geq 79$ year)) & 3 & 29 \\
\hline D (Two or more falls in the previous year) & 4 & 43 \\
\hline AB & 4 & 45 \\
\hline $\mathrm{AC}$ & 5 & 47 \\
\hline $\mathrm{BC}$ & 5 & 48 \\
\hline AD & 6 & 62 \\
\hline $\mathrm{BD}$ & 6 & 63 \\
\hline$C D$ & 7 & 65 \\
\hline$A B C$ & 7 & 66 \\
\hline$A B D$ & 8 & 78 \\
\hline ACD & 9 & 79 \\
\hline $\mathrm{BCD}$ & 9 & 80 \\
\hline$A B C D$ & 11 & 90 \\
\hline
\end{tabular}

$\mathrm{PP}=$ predicted probability

The predicted probability of a new fall can be estimated according the formula: $\mathrm{P}=1 /[1+\mathrm{e}-\mathrm{g}]$

Where $g=\left(\beta 0+\beta 1 x^{1}+\beta 2 x^{2}+\beta 3 x^{3}+\beta 4 x 4\right), \beta 0$ is the constant and $\beta 1, \beta 2, \beta 3$ and $\beta 4$, are the regressi coefficients for each of the predictors $x 1, \times 2, \times 3$ and $\times 4$, each coded as 1 if present and 0 if absent in a pers Example: a 85 year old person, with no recurrent falls in the previous year, a FHI score of 30 and having a joint disorder, has a probability of a new fall within one year of: $1 /[1+\mathrm{e}-(-1.75+0.87+0.79+0.76)]=0.66$ 
Table 5 - Sensitivity, specificity and positive and negative predictive values of the fall risk screening tool for new falls at different cut-off points

\begin{tabular}{llccccc}
\hline $\begin{array}{l}\text { Cut-off points in total } \\
\text { risk score }\end{array}$ & Participant in high-risk group (\%) & SE & SP & $\Sigma$ & PV+ & PV- \\
\hline 0 versus $\geq 2$ & & $(\%)$ & $(\%)$ & $(\%)$ & $(\%)$ & $(\%)$ \\
$0-2$ versus $\geq 3$ & 82.6 & 94.2 & 34.6 & 128.8 & 54.4 & 87.8 \\
$0-3$ versus $\geq 4$ & 66.7 & 83.7 & 52.9 & 136.6 & 59.5 & 79.7 \\
$0-4$ versus $\geq 5$ & 41.3 & 79.1 & 59.6 & 138.7 & 61.8 & 77.5 \\
$0-5$ versus $\geq 6$ & 42.9 & 67.4 & 73.1 & $140.5^{*}$ & 67.4 & 73.1 \\
$0-6$ versus $\geq 7$ & 26.7 & 60.5 & 76.9 & 137.4 & 68.4 & 70.2 \\
$0-7$ versus $\geq 8$ & 21.0 & 39.5 & 88.5 & 128.0 & 73.9 & 63.9 \\
$0-8$ versus $\geq 9$ & 13.2 & 32.6 & 95.2 & 127.8 & 84.9 & 63.1 \\
$0-10$ versus $\geq 11$ & 4.8 & 19.8 & 97.1 & 116.9 & 85.0 & 59.4 \\
\hline
\end{tabular}

$\mathrm{SE}=$ sensitivity; $\mathrm{SP}=$ specificity; $\Sigma=$ sum of sensitivity and specificity; $\mathrm{PV}+=$ positive predicted value; PV- $=$ negative predicted value; ${ }^{*}=$ maximum $\Sigma$

Figure 1 shows a ROC curve for the current screening instrument. The AUC is 0.77 (95\% $\mathrm{Cl}$ : 0.70-0.83) indicating that when we draw at random one person out of the low risk group and one person out of the high-risk group, in $77 \%$ of the cases we were able to discriminate correctly between the two groups.

\section{Fall risk screening tool}

Based on the risk model it is possible to develop a simple fall risk screening tool. The four predictors comprising the screening tool can be easily assessed. Information with regard to age, recurrent falls in the previous year, and whether the faller suffers from joint disorders can be gathered by means of three simple questions. To assess handicaps associated with the last sustained fall a self-administered questionnaire has to be completed. This can easily be done by the patient and/or nurse (practitioner) at the A\&E department or GP when a faller presents with an in injury resulting from a fall. With this information it can easily be determined what risk a patient runs for falling again (table 4). 


\section{ROC Curve}

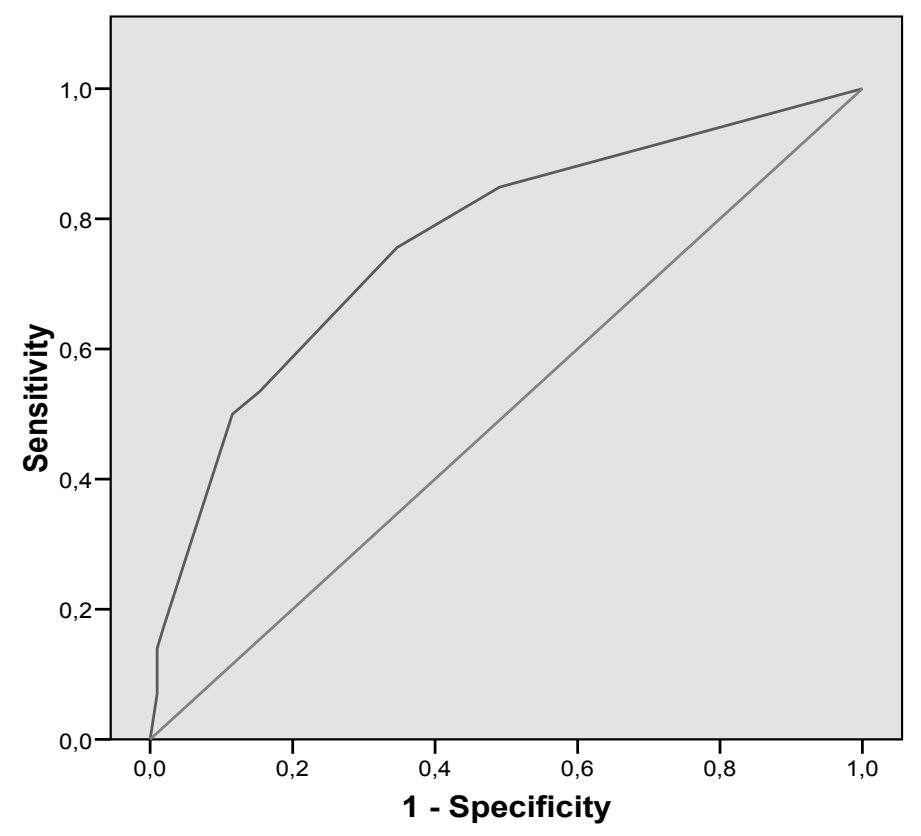

Figure 1 - ROC curve for the screening tool to predict a new fall; AUC $=0.71(0.70-0.83)$

\section{DISCUSSION}

\section{Main results}

The current explorative study resulted in 15 risk factors which contribute to the prediction of a new fall among community-dwelling people aged 65 years or over who have visited the A\&E department of a hospital with the consequences of a fall. Based on these 15 risk factors a screening tool was developed which predicts the risk of sustaining a new fall among a group of community-dwelling injurious fallers aged 65 years of over by 4 predictors which can be easily assessed: age ( $\geq 79$ year), two or more falls in the previous year, experiencing handicaps associated with the last sustained fall(s) ( $F H I \geq 24$ ), and having joint disorders. After assessing whether these predictors are present or not, it can easily be determined what risk a patient runs for falling again. The total risk score of the fall risk screening tool ranges 
from 0 to 11 , which corresponds to a probability ranging from $15 \%$ to $90 \%$. The optimal cut-off point of the fall risk screening tool is reached at a total risk score of 5 . For this cut-off point, the percentage of persons that were classified correctly as faller (sensitivity) was $67.4 \%$ $(n=68)$. The percentage of persons who did not fall again that were classified correctly as non faller (specificity) was $73.1 \%(n=76)$. The results indicate that the developed fall risk screening tool in this group can improve the efficiency to select people at high-risk for sustaining a new fall.

\section{Methodological considerations}

Some limitations of the present study should be discussed. First, because this study was not initially intended to construct a screening tool, we did not measure mobility impairment, which is generally considered to be an important risk factor for falls $(2,18,34-40)$. However we measured a number of mobility related variables (such as mobility related questions of het GARS, FAl en EuroQol) (25-28). Therefore it should be doubted whether we would have achieved a more sensitive screening tool when we had included a measure for mobility impairment. Second, the number of participants used in the analyses of the current study was relatively small $(n=190)$, because only those participants with complete follow-up data on falls were included. It might be that by including only those persons with complete data in the analyses, we excluded persons with a high-risk for falling again. This assumption may be correct since those persons were more ADL dependent, reported more health complaints, and had more problems with walking. However, repetition of the analysis including also people with incomplete data (for whom at least 9 of the 12 months of the fall data were available, missing data were replaced by the individual mean of valid data, $n=258$ ), does provide the same results. However, further validation of our fall risk screening tool in other populations of injurious fallers is suggested. In addition, the role of mobility impairments as predictor of future falls may be investigated more extensively.

\section{Implications for practice}

As stated, the optimal cut-off point of the fall risk screening tool is reached at a total risk score of 5 . However, when we decrease or increase the cut-off point the sensitivity respectively increases or decreases and the specificity respectively decreases or increases. The decision whether to choose for a higher sensitivity and lower specificity or vice versa depends on what seems to be most acceptable: missing people at risk or including people without risk in the 
high risk group. The solution depends among others from the available (effective) prevention strategies and the related costs.

According to the present model, healthcare workers on an A\&E department or GP's should primarily focus on four risk factors for sustaining a new fall among persons who experienced an injurious fall: age ( $\geq 79$ year), two or more falls in the previous year, experiencing handicaps associated with previous fall(s) fall(s) ( $F H I \geq 24)$, and having joint disorders. Based on the outcome of this risk assessment patients should be offered an effective and feasible fall prevention programme. 


\section{REFERENCES}

1. Wyman JF, Croghan CF, Nachreiner NM, Gross CR, Hatch Stock H, Talley K, et al. Effectiveness of Education and Individualized Counseling in Reducing Environmental Hazards in the Homes of Community-Dwelling Older Women. J Am Geriatr Soc. 2007;55:1548-56.

2. Rubenstein LZ. Falls in older people: epidemiology, risk factors and strategies for prevention. Age Ageing. 2006 Sep;35 Suppl 2:ii37-ii41.

3. Gates S, Lamb SE, Fisher JD, Cooke MW, Carter YH. Multifactorial assessment and targeted intervention for preventing falls and injuries among older people in community and emergency care settings: systematic review and meta-analysis. Bmj. 2008 Jan 19;336(7636):130-3.

4. Hauer K, Lamb SE, Jorstad EC, Todd C, Becker C. Systematic review of definitions and methods of measuring falls in randomised controlled fall prevention trials. Age Ageing. 2006 Jan;35(1):5-10.

5. Kannus P, Sievanen H, Palvanen M, Jarvinen T, Parkkari J. Prevention of falls and consequent injuries in elderly people. Lancet. 2005 Nov 26;366(9500):1885-93.

6. Chang JT, Morton SC, Rubenstein LZ, Mojica WA, Maglione M, Suttorp MJ, et al. Interventions for the prevention of falls in older adults: systematic review and meta-analysis of randomised clinical trials. Bmj. 2004;328(7441):680.

7. Gillespie LD, Gillespie WJ, Robertson MC, Lamb SE, Cumming RG, Rowe BH. Interventions for preventing falls in elderly people. Cochrane Database Syst Rev. 2003(4):CD000340.

8. Stalenhoef PA, Diederiks JP, Knottnerus JA, de Witte LP, Crebolder HF. The construction of a patient record-based risk model for recurrent falls among elderly people living in the community. Fam Pract. 2000; 17(6):490-6.

9. Bueno Cavanillas A, Padilla Ruiz F, Jimenez Moleon JJ, Peinado Alonso CA, Galvez Vargas R. Risk factors in falls among the elderly according to extrinsic and intrinsic precipitating causes. Eur J Epidemiol. 2000; 16(9):849-59.

10. Cesari M, Landi F, Torre S, Onder G, Lattanzio F, Bernabei R. Prevalence and risk factors for falls in an older community-dwelling population. J Gerontol A Biol Sci Med Sci. 2002 Nov;57(11):M722-6.

11. Berg WP, Alessio HM, Mills EM, Tong C. Circumstances and consequences of falls in independent community-dwelling older adults. Age Ageing. 1997;26(4):261-8.

12. Mackenzie L, Byles J, Higginbotham N. A prospective community-based study of falls among older people in Australia: frequency, circumstances, and consequences. OTJR. 2002;22(4):143-52.

13. Masud T, Morris, R.O. Epidemiology of falls. Age Ageing. 2001;30 (S4):3-7. 
14. Lach HW, Reed, T., Arfken, C.L., Miller, J.P., Paige, G.D. Falls in the elderly: reliability of a classification system. Journal of the American Geriatrics Society. 1991;39:197-202.

15. Hill K, Schwarz J, Flicker L, Carroll S. Falls among healthy, community-dwelling, older women: a prospective study of frequency, circumstances, consequences, and prediction accuracy. Australian and New Zealand Journal of Public Health. 1999;23:41-8.

16. Lord SR, Menz HB, Sherrington C. Home environment risk factors for falls in older people and the efficacy of home modifications. Age Ageing. 2006 Sep;35 Suppl 2:ii55-ii9.

17. Gillespie L. Preventing falls in elderly people. Bmj. 2004 Mar 20;328(7441):653-4.

18. Todd C, Skelton D. What are the main risk factors for falls among older people and what are the most effective interventions to prevent falls? Copenhagen: WHO Regional Office for Europe (Health Evidence Network report); 2004 March 2004. Report No.: E82552.

19. Ganz DA, Bao Y, Shekelle PG, Rubenstein LZ. Will my patient fall? JAMA. 2007 Jan 3;297(1):77-86.

20. Hendriks MR, van Haastregt JC, Diederiks JP, Evers SM, Crebolder HF, van Eijk JT. Effectiveness and costeffectiveness of a multidisciplinary intervention programme to prevent new falls and functional decline among elderly persons at risk: design of a replicated randomised controlled trial [ISRCTN64716113]. BMC Public Health. 2005 Jan 14;5:6.

21. Hendriks MR, Bleijlevens MH, van Haastregt JC, Crebolder HF, Diederiks JP, Evers SM, et al. Lack of effectiveness of a multidisciplinary fall-prevention program in elderly people at risk: a randomized, controlled trial. J Am Geriatr Soc. 2008 Aug;56(8):1390-7.

22. Bleijlevens $M H$, Hendriks MR, van Haastregt JC, van Rossum E, Kempen GI, Diederiks JP, et al. Process factors explaining the ineffectiveness of a multidisciplinary fall prevention programme: a process evaluation. BMC Public Health. 2008 Sep 24;8(1):332.

23. Rai GS, Kiniorns M, Wientjes H. Falls Handicap Inventory (FHI)--an instrument to measure handicaps associated with repeated falls. J Am Geriatr Soc. 1995;43(6):723-4.

24. CBO KvdG. Richtlijn Preventie van valincidenten bij ouderen. Alphen aan den Rijn: Van Zuiden Communications B.V.; 2004.

25. Schuling J, de Haan R, Limburg M, Groenier KH. The Frenchay Activities Index. Assessment of functional status in stroke patients. Stroke. 1993 Aug;24(8):1173-7.

26. Kempen GI, Miedema I, Ormel J, Molenaar W. The assessment of disability with the Groningen Activity Restriction Scale. Conceptual framework and psychometric properties. Soc Sci Med. 1996 Dec;43(11):1601-10.

27. Brooks R. Euroqol:the current state of play. Health policy. $1996 \mathrm{Jul} ; 37(1): 53-72$. 
28. Drummond MF, Sculpher MJ, Thorrance GW, O'Brien BJ, Stoddart GL. Methods for the Economic Evaluation of Health Care Programmes. Third ed. New York: Oxford University Press; 2005.

29. Spinhoven P, Ormel J, Sloekers PP, Kempen GI, Speckens AE, Van Hemert AM. A validation study of the Hospital Anxiety and Depression Scale (HADS) in different groups of Dutch subjects. Psychol Med. 1997 Mar;27(2):363-70.

30. Crawford JR, Henry JD, Crombie C, Taylor EP. Normative data for the HADS from a large non-clinical sample. Br J Clin Psychol. 2001;40(Pt 4):429-34.

31. Kellogg International Work Group on the Prevention of Falls by the Elderly. The prevention of falls in later life. Dan Med Bull. 1987;34 Supp/ 4:1-24.

32. Hosmer DW, Taber S, Lemeshow S. The importance of assessing the fit of logistic regression models: a case study. Am J Public Health. 1991 Dec;81(12):1630-5.

33. Apfel CC, Laara E, Koivuranta M, Greim CA, Roewer N. A simplified risk score for predicting postoperative nausea and vomiting: conclusions from cross-validations between two centers. Anesthesiology. 1999 Sep;91(3):693-700.

34. Campbell AJ, Borrie MJ, Spears GF. Risk factors for falls in a community-based prospective study of people 70 years and older. J Gerontol. 1989;44(4):M112-7.

35. Davis JW, Ross PD, Nevitt MC, Wasnich RD. Risk factors for falls and for serious injuries on falling among older Japanese women in Hawaii. J Am Geriatr Soc. 1999;47:792-98.

36. Luukinen H, Koski K, Honkanen R, Kivela SL. Incidence of injury-causing falls among older adults by place of residence: a population-based study. J Am Geriatr Soc. 1995 Aug;43(8):871-6.

37. Nevitt MC, Cummings, S.R., Kidd, S., Black, D. Risk factor for recurrent nonsyncopal falls. JAMA. 1989;261:2663-8.

38. O'Loughlin J, Robitaille Y, Bolvin J-F, Suissa S. Incidence of and risk factors for falls and injurious falls among the community-dwelling elderly. Am J Epidemiol. 1993;137:342-54.

39. Russell MA, Hill KD, Blackberry I, Day LL, Dharmage SC. Falls risk and functional decline in older fallers discharged directly from emergency departments. J Gerontol A Biol Sci Med Sci. 2006 Oct;61(10):1090-5.

40. Stalenhoef PA, Diederiks JP, Knottnerus JA, Kester AD, Crebolder HF. A risk model for the prediction of recurrent falls in community-dwelling elderly: a prospective cohort study. J Clin Epidemiol. 2002 Nov;55(11):1088-94. 


\section{GENERAL DISCUSSION}

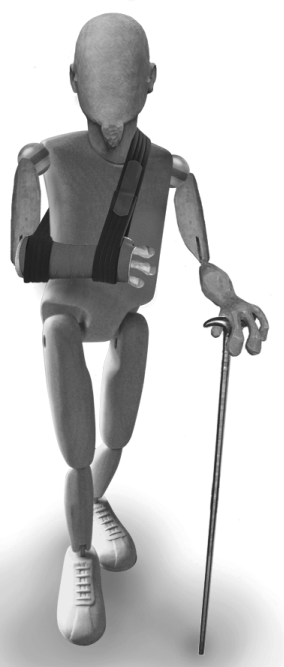




\section{INTRODUCTION}

Falls and their consequences are a major health problem for both individuals and society. Fall prevention is necessary since falls are associated with increased mortality and morbidity. In addition, falls are a burden to older people and their families. Falls, especially injurious falls, are also associated with an increase in healthcare utilization. In view of the individual and societal impact of falls, the need to decrease the number of falls and their adverse consequences is obvious.

In the Netherlands, at the start of our research project in 2002, there was a clear need for effective fall prevention programmes among older people. In the preceding year (2001), Gillespie and colleagues had concluded in their systematic review that health professionals considering fall prevention programmes should consider health screening of older persons at risk for falling, followed by interventions targeted at both intrinsic and extrinsic risk factors of individuals (1). Furthermore, in the years 2000 and 2001, guidelines for the prevention of falls were introduced in the UK and USA respectively, recommending that multi-component fall prevention programmes aimed at high risk populations of older persons should be performed $(2,3)$. An example of such a multi-component fall prevention programme was a programme developed by Close and colleagues (4). This multidisciplinary fall prevention programme consisted of a medical and occupational-therapy assessment aimed at elderly people attending the Accident and Emergency (A\&E) department with injuries resulting from a fall. The programme had been evaluated in a randomised controlled trial (PROFET) in the UK (4). The PROFET study demonstrated that this multidisciplinary intervention, applied to people at risk, was highly effective in reducing the number of recurrent falls and associated injuries [4]. However, proven effectiveness of this programme in an experimental setting in the UK is no guarantee that it will be effective when implemented in other healthcare settings. Therefore, we decided to evaluate this successful British programme within a Dutch healthcare setting, after adapting it for use in the Dutch setting $(5,6)$.

The main objective of the current study is to assess the effectiveness and feasibility of a medical and occupational therapy programme to prevent falls and functional decline among elderly people at risk, compared to the usual healthcare in the Netherlands. The study comprised five parts, whose objectives are described below: 
1. To assess the effectiveness of a multifactorial medical and occupational-therapy fall prevention programme among elderly people at risk, in terms of falls, functional decline, and a number of secondary outcomes.

2. To assess the feasibility of this fall prevention programme for elderly people as well as the medical and paramedical practitioners who performed the assessments.

3. To assess the role of the occupational-therapy part of the fall prevention programme in preventing new falls and functional decline.

4. To assess whether it is possible to establish a classification of injurious fall types based on fall location and activity up to the moment of the fall.

5. To assess which risk factors predict new falls among a group of community-dwelling injurious fallers aged 65 years or over, in order to achieve a better selection of older people at high risk for falling.

In this chapter, the main findings of the studies described in the previous chapters of this thesis will be discussed. This will be followed by a consideration of the current state of the art with regard to fall prevention research and some theoretical and methodological considerations. Finally, implications for practice and future research will be presented.

\section{MAIN FINDINGS}

\section{Effects of the programme}

The RCT showed that the multifactorial fall prevention programme had no effect on the primary outcome measures falls (i.e. falls, recurrent falls, injurious falls, and time to the first fall) and daily functioning, nor on the secondary outcome measures (recuperation from the fall, health complaints, perceived health, activities of daily living (ADL), and instrumental activities of daily living (IADL), disability, mental health, fear of falling, activity avoidance, social participation, and quality of life). The per-protocol analyses in which the subjects who received the complete intervention were compared with the control group, and the complete case analyses including those participants with complete data, resulted in comparable outcomes. 


\section{Feasibility of the programme}

The process evaluation revealed that the overall programme was judged acceptable and feasible for both practitioners and participants. Moreover, the results of this study show that the programme was largely performed according to protocol. Although a large majority of the participants reported that they had benefited from the programme, the number of referrals and recommendations ensuing from the medical assessments was smaller than expected (0.58 per participant), especially when compared to the recommendations ensuing from the occupational-therapy assessments (3.57 per participant). Participants' self-reported compliance with the advice to contact their general practitioner (GP) to be informed about the recommendations and referrals resulting from the medical assessment was low to moderate. However, participants who did call their GPs and were informed by their GPs with regard to referrals and recommendations reported reasonable to good compliance with these referrals and recommendations.

\section{In-depth analysis of the occupational-therapy part of the programme}

The in-depth analysis of the occupational-therapy programme revealed that the recommendations resulting from the occupational-therapy assessment mainly comprised instructions about behaviour change and services and assistive devices provided under the Services for the Disabled Act (WVG). The instructions about behaviour change were most of the time confined to recommendations to reduce risky behaviour. These recommendations were made during the home visit by the occupational therapist (OT) but actual implementation was not checked by the therapist afterwards. Although, two thirds of the recommendations regarding services and assistive devices covered by the WVG were actually implemented as reported by the participants, the implementation of these recommendations took on average almost half a year. These findings resulted in a number of recommendations to improve the occupational-therapy part of the programme. First, we recommend the realization of a reduction in the time between the home visit and the actual implementation of recommendations. Second, participants should be supported by the OT to implement recommended changes. Third, the OT should use theory-based techniques to stimulate behaviour change and use follow-up visits to promote maintenance of the desired behaviour. 


\section{Circumstances under which falls occur}

The exploratory study on the circumstances under which injurious falls occur revealed that injurious falls in this study can be classified into specific fall types based on the combination of fall location and activity up to the moment of a fall. We identified four injurious fall types:

1. Indoor falls in the hall and bathroom, predominantly during lavatory visits.

2. Indoor falls at locations other than the hall and bathroom, predominantly during ADL.

3. Outdoor falls near the home (garden, access path), predominantly during IADL.

4. Outdoor falls away from home, occurring predominantly during walking, cycling, and shopping for groceries.

There seems to be a difference in fall location and activity up to the moment of the fall between the younger and more active older persons, who still go outdoors, and the more frail older people who tend to stay indoors. Those persons who fell outdoors predominantly reported an extrinsic cause of their fall, whereas those persons who fell indoors reported an intrinsic cause. We found no difference between the four injurious fall types in terms of the consequences of the fall, except for avoidance of activity after the fall (persons who fell outside during mobility related activities were less avoidant).

\section{Risk factors for new falls among injurious fallers}

The exploratory study which assessed which risk factors predict new falls among a group of community-dwelling injurious fallers aged 65 years or over yielded 15 risk factors which contribute to the prediction of a new fall. The 15 risk factors have been used to develop a screening tool which predicts the risk of sustaining a new fall among community-dwelling injurious fallers aged 65 years or over. This resulted in a tool comprising four predictors which can be easily assessed: age ( $\geq 79$ years), two or more falls in the previous year, experiencing handicaps associated with the previous fall(s) (score on the Falls Handicap Inventory $\geq 24$ ), and having joint disorders. Assessing the presence of these predictors in injurious fallers helps to predict new falls. The area under the curve (AUC) is 0.77 (95\% $\mathrm{Cl}$ : $0.70-0.83$ ) indicating that in $77 \%$ of the cases we were able to discriminate correctly between those who experienced a new fall and those who did not. 


\section{CONSIDERATIONS}

Some theoretical and methodological issues should be considered in order to facilitate interpretation of the results of the studies described in this thesis. First we relate our results to the present state of the art in the field of fall prevention. Second we discuss possible explanations of the lack of effectiveness of our intervention, and third we discuss some methodological issues of our studies.

\section{Fall prevention: the state of the art}

In the past decades several systematic reviews and meta-analyses were performed to assess the effects of fall prevention interventions (7-12). In 2004, Chang and colleagues (7) reported in their meta-analyses that multifactorial fall risk and management programmes were effective in reducing both the risk of falling (proportion of fallers) and the monthly rate of falling for people at risk of falling (7). In addition, they found that exercise programmes were effective in reducing the risk of falling. A subsequent meta-analysis by Weatherall in 2004 (11) showed that there was moderate evidence of efficacy of fall prevention particularly for multiple intervention strategies. The meta-regression done by Campbell and colleagues (10) showed that multifactorial fall prevention interventions were effective in reducing the rate of falls among selected populations. In addition, Campbell and colleagues found that interventions addressing a single risk factor are as effective in reducing falls as interventions with multiple components (10). In 2008, Gates and colleagues reported on a systematic review of multifactorial assessment and intervention programmes. They agreed with the previous mentioned systematic reviews and meta-analyses that fall risk assessment and intervention appear to be an attractive solution for fall prevention. However, Gates and colleagues only found evidence of a significant reduction in the number of fallers in higher intensity interventions (interventions that actively provide treatments directly aimed at reducing risk factors). They suggest that multifactorial interventions that provide direct treatment, rather than only referral and knowledge, may be more effective (higher intensity subgroup risk ratio 0.84 (95\% Cl: 0.74 to 0.96) (9). Very recently (July 2009), Gillespie and colleagues have updated their systematic review (12). Gillespie and colleagues conclude that multifactorial interventions integrating assessment with individualised intervention, usually involving a multi-professional team, are effective in reducing the number of falls but not in reducing the proportion of persons sustaining a fall, $(0.75,95 \% \mathrm{Cl}$ : 0.65 to 0.86$)$ 
and $(0.95,95 \% \mathrm{Cl}: 0.88$ to 1.02$)$ respectively (12). In contrast to Gates and colleagues they found no evidence that assessment and direct intervention (high intensity) are more effective than assessment and referral/knowledge alone. They also found no evidence that multifactorial interventions are more effective in participants selected as being at higher risk of falling (12). Five systematic reviews came to the conclusion that multifactorial risk assessment and management programmes are likely to be effective in reducing the number of falls and/or the proportion of fallers among older people (7-12). However, the evidence on which these conclusions are based is moderate and sometimes conflicting. This also concerns those multifactorial programmes which comprise direct, high intensity treatments. The outcomes reported by Gillespie and colleagues (12) regarding the subgroup analysis by intensity of the intervention failed to confirm the finding of Gates and colleagues (9), which is possibly due to differences in inclusion criteria and the number of completed trials available for inclusion in both reviews.

\section{Why was our fall prevention programme not effective?}

As stated before, the multifactorial fall prevention programme described in this thesis was based on a multi-component programme to prevent falls among elderly people developed by Close and colleagues (4). The programme was also in line with the recommendations resulting from two fall prevention guidelines from the UK and USA $(3,13)$ and the results of the systematic review done by Gillespie et al in 2003 (1). At the start of our project this was the best available evidence regarding how to prevent falls among older persons who attended the A\&E department as a result of a fall. Despite the promising results from the PROFET study in the UK (4), our effect evaluation showed that the adapted Dutch version of this programme had no favourable effects on the number of fallers in the Netherlands (14). Therefore, a number of aspects of our multifactorial fall prevention programme need to be considered in order to gain insight into the possible reasons for the lack of effectiveness: a) the adaptations made to the intervention in order to integrate it into the Dutch health system; b) the small numbers of recommendations and referrals resulting from the medical assessment; and c) the content of the intervention programme. 


\section{GENERAL DISCUSSION}

a) The adaptations made to the intervention

In order to facilitate future implementation of the intervention programme in Dutch regular healthcare, we involved the GPs in our intervention programme and decided that services and assistive devices recommended by the occupational therapist should be provided according to the WVG, which was implemented by the municipal authorities of the research region (15). With hindsight, involving the participants' GPs and the fact that recommended services had to be provided according to the WVG had several negative consequences for the effectiveness and feasibility of our programme.

First, involving the GP resulted in more time expiring between the moment the patients received referrals and/or recommendations resulting from the medical assessment and the actual implementation of those referrals and/or recommendations. It may therefore be possible that participants already sustained a new fall before the risk factors assessed during the medical assessment were addressed.

Second, participants had to call their GPs to be informed about the results of the medical assessment. However, half of the participants did not call their GPs and therefore were not informed about possible recommendations and/or referrals.

Third, in line with this, it is possible that the GPs did not follow up all the recommendations made by the geriatrician who was responsible for the medical assessment. A possible reason for not following the geriatrician's suggested referrals and/or recommendations may be the GP did not agree with the recommendation(s). Involving the GP in the intervention programme turned out to be an inefficient procedure and is likely to have contributed to the lack of effectiveness of our programme.

Finally, the decision that services and assistive devices recommended by the OT should be provided according to the WVG resulted in a relatively long period (on average 6 months) between the home visit by the OT and the actual implementation of the recommended services and assistive devices. Due to this time delay, it is possible that persons participating in our study sustained a new fall before the risk factors which were assessed during the medical and occupational-therapy assessments had been addressed. 
b) The small numbers of recommendations and referrals

The lack of effectiveness of our programme may also be explained by the relatively small number of referrals and recommendations ensuing from the medical assessment. This may be the result of several factors.

First it is possible that we did not select the high-risk group we intended to select. A highrisk group for falling is often identified by a history of recurrent falls, a fall requiring medical attention, or an abnormality of gait, balance, or both $(2,16)$. The population selected for our study meets a least two of these criteria, since the participants attended the A\&E department of a hospital and had a history of recurrent falls. However, the process evaluation revealed that the practitioners who implemented the programme found that the group we selected for participation also comprised a substantial number of persons who were not at high risk for falls (17). The argument that the small number of referrals and recommendations may be related to this factor seems to be supported by the fact that the population in our trial was somewhat younger compared with that in the PROFET study of Close and colleagues (74.9 versus 78.2 years). In addition, only a small number of participants died during our study compared with the PROFET study ( $n=6,2 \%$ versus $n=46,12 \%$ ). Finally, none of our participants moved into a home for the elderly or nursing home during the study in contrast to 36 participants (18\%) in the PROFET study. Based on this, it seems that our population was in a better condition, which is likely to be related to a lower risk of falls. However, when we observe the recurrent falls sustained by persons in our control group during the trial period, the number of (recurrent) falls is comparable to the number of (recurrent) falls reported in the study of Close and colleagues and other comparable studies $(4,18,19)$.

Second, the small number of referrals and recommendations resulting from the assessments may be explained by the possibility that our population already received sufficient care, meaning that possible risk factors were assessed and addressed during regular care. This would mean that our intervention does not add extra treatment possibilities to regular care, which has resulted in a lack of contrast between our intervention and regular care. However, the fact that the number of recurrent falls in our control group was comparable to that in the study of Close and other studies does not indicate that usual care in our study was better in preventing falls than usual care in those other studies. 
c) The content of the intervention programme.

Finally the lack of effectiveness of the intervention programme may be explained by the fact that the programme did not include an exercise component. Several systematic reviews revealed that exercise is an intervention component with proven effectiveness $(7,8,12,20)$. The process evaluation revealed that only four of our participants received a recommendation that may be related to exercise (referral to physiotherapist).

\section{Methodological issues}

Below we discuss a number of methodological issues regarding the studies presented in this thesis.

First, due to the design of our study it is possible that the GPs involved in our trial had both intervention and control persons in their medical practices. Therefore, our intervention programme may have influenced the regular treatment of patients in the control group. However, the referrals and recommendations for the patients in the intervention group resulted from an individualised assessment and were tailor made. Moreover, the GPs did not know which patients participated in the control group of our study. Therefore, we assume that a possible contamination effect is negligible.

Second, with regard to the process evaluation (Chapter 3), the outcomes may have been influenced by socially desirable answers given by both participants and practitioners involved. Although we have tried to avoid desirable answers by emphasizing that their answers would not affect their future treatment or work, we cannot exclude the possibility that both participants and practitioners suppressed some negative aspects they perceived. We also did not involve the GPs in our process evaluation. Since we did not gather data directly from the GPs, we do not have information regarding their opinions about the fall prevention programme and their motives for not complying with all recommendations and/or referrals resulting from the medical assessments.

Third, according to the in-depth analysis of the occupational-therapy part of our programme (Chapter 4), the assessment resulted in 301 recommendations with regard to behaviour change. However, recommendations on behaviour change were limited to pointing out 
a person's fall-related risk behaviour during one home visit and suggesting a change in behaviour to reduce their fall risk in the future. It is doubtful whether this single contact is sufficient to result in an actual change of the behaviour as recommended (9)

Fourth, regarding our study of the relationship between location and activity in injurious falls (Chapter 5), it should be mentioned that all persons in our sample attended the A\&E department of a hospital with the consequences of injurious falls. We did not select persons who visited their own GPs and those persons who did not seek medical attention at all for the consequences of their falls. So, we only included a group with serious injuries resulting from a fall. This may have compromised the representativeness of our study population for this part of our study. Furthermore, it should be noted that the analyses are data-driven, meaning that no a priori hypotheses were formulated. The homogeneity analysis by means of alternating least squares (HOMALS) was allowed to determine the best partitioning between the four fall types. Therefore, the results should be replicated in other populations.

Finally, it should be mentioned that our study assessing risk factors to predict new falls among a group of community-dwelling injurious fallers aged 65 years or over (Chapter 6 ) was initially not intended to construct a screening tool. Therefore, we did not measure mobility impairment, which is generally considered to be an important risk factor for falls $(18,21$ 28). However, mobility impairment was assessed by means of self-report using a number of mobility related variables (such as mobility related questions of the Groningen Activity Restriction Scale (GARS), the Frenchay Activities Index (FAI) and the European Quality of Life instrument (EuroQol)) (29-32). Therefore it is doubtful whether we would have achieved a more sensitive screening tool if we had included a measure for mobility impairment. 


\section{CONCLUSION AND RECOMMENDATIONS}

\section{Conclusion}

Our fall prevention programme comprising medical and occupational-therapy assessment did not have favourable effects on falls and daily functioning among older persons of 65 years and over who had sustained an injurious fall. Nevertheless, the programme proved to be feasible and acceptable for both the participants and the practitioners who performed the medical and occupational-therapy assessments. There still remains a great need for effective fall prevention strategies, because falls bring about substantial mortality, morbidity, and suffering for older people and their relatives, and considerable healthcare costs (28). Based on the findings and experiences from the current study, we do not recommend implementation of our fall prevention programme in its current form. It is of utmost importance to improve several aspects of the programme and to assess whether these adaptations will result in greater effectiveness and efficiency.

\section{Recommendations for clinical practice}

We recommend three major adjustments to improve the efficiency and effectiveness of our fall prevention programme: a) adding an exercise component; b) improving the selection of the target population; and c) decreasing barriers to implementation.

\section{a) Adding an exercise component}

We recommend the addition of an exercise component to our multifactorial fall prevention programme, as this has proven to be the most effective single fall prevention intervention component $(7,33)$.

b) Improving the selection of the target population.

In order to increase the efficiency of our programme it should be aimed at persons who are at relatively high risk of sustaining a new fall. We therefore recommend using the screening tool we developed (Chapter 5).

\section{c) Decreasing barriers to implementation}

In order to improve fall prevention in clinical practice, possible barriers to implementation should be overcome (34-37). These barriers can be subdivided into logistical and systematic factors $(34,38)$, patient factors $(34,35)$, and physician/GP factors $(35,37,39)$. Below these three factors are described in more detail. 


\section{Logistical and systematic factors}

The present study revealed that the time gap between a person attending the A\&E department after a fall and actual action taken in order to prevent future falls may have negatively influenced the effectiveness and efficiency of our intervention programme. It therefore seems very important that the factors causing this time delay are addressed in the future. We recommend performing the medical assessment within two weeks after attending the A\&E department for those directly discharged home, and around discharge for those admitted to hospital after the fall. Moreover, the occupational-therapy assessment should be performed within two weeks after the patient is discharged home. To further increase the efficiency, the geriatrician under whose guidance the medical assessment is performed should be permitted to refer patients directly to relevant services instead of having the GP implement the referrals. To inform the GP, the geriatrician and occupational therapist should send the GP a comprehensive report on the outcomes of the assessments and the actions already taken. This would allow the GPs to continue and coordinate the fall prevention measures initiated or implemented by the geriatrician and occupational therapist. In order to realise these recommended adaptations, it is important that all professionals directly or indirectly involved in the implementation of the programme have regular contact in order to facilitate successful implementation of the adapted programme. Furthermore, integrating the adapted programme in a falls clinic could also be considered, because within a falls clinic the lines between the different disciplines are short and referrals can be made directly. To our knowledge, there have been a few studies that reported on the outcomes of falls clinics $(40,41)$. Overall, these studies have indicated substantial reductions (between 35\% and $77 \%$ ) in falls in high falls-risk populations $(40,41)$. However, further research to assess (cost-) effectiveness is needed.

\section{Patient factors}

Patient compliance with the referrals and recommendations resulting from both the medical and occupational-therapy assessments needs to be improved. To increase compliance with the referrals and recommendations, patients should be supported over a period of time to comply with the recommended referrals and to achieve the recommended changes. Referrals and recommendations resulting from the medical assessment should be given extra attention during subsequent consultations. For the recommendations resulting from the occupationaltherapy assessment, follow-up visits should be performed to check whether the services and 
assistive devices are actually implemented and are being correctly used. Furthermore, the occupational therapists should use theory-based techniques to stimulate behaviour change and use follow-up visits to encourage behaviour change and promote maintenance of the desired behaviour.

\section{Physician/GP factors}

In the Netherlands, in the education of (future) physicians/GPs, more attention should be paid to the aetiology and prevention of falls in older persons. Fall prevention should be increasingly integrated in regular healthcare. Guidelines for the prevention of falls should be formulated and implemented based on the current state of the art. This integration of fall prevention in regular care may increase the chances of successful implementation of effective (evidence based) fall prevention strategies in the future.

\section{Recommendations for future research}

First, we recommend exploration of whether our conclusion that injurious fallers can be subdivided into four injurious fall types can be confirmed in other populations of injurious fallers.

Second, further validation of our fall risk screening tool is needed to confirm our hypothesis that this tool can distinguish between injurious fallers with low and high fall risks.

Third, we recommend that in the Netherlands fall prevention research should focus more on implementation research, in order to gain additional insight into how to disseminate successful fall prevention strategies in regular healthcare. The reason for this is that in the past several fall prevention strategies which were effective in other healthcare settings (4, $19,42-44)$, turned out to be ineffective in the Netherlands $(14,45,46)$. We need to create additional insight into factors explaining this lack of effectiveness. It is therefore important to include a detailed process-evaluation in fall prevention studies in the Netherlands and to pay more attention to the qualitative evaluation of implementation processes in the field of fall prevention in the Netherlands. 


\section{REFERENCES}

1. Gillespie LD, Gillespie WJ, Robertson MC, Lamb SE, Cumming RG, Rowe BH. Interventions for preventing falls in elderly people. Cochrane Database Syst Rev. 2001(3):CD000340.

2. Guideline for the prevention of falls in older persons. American Geriatrics Society, British Geriatrics Society, and American Academy of Orthopaedic Surgeons Panel on Falls Prevention. J Am Geriatr Soc. 2001 May;49(5):664-72.

3. Feder G, Cryer C, Donovan S, Carter Y. Guidelines for the prevention of falls in people over 65. The Guidelines' Development Group. Bmj. 2000 Oct 21;321(7267):1007-11.

4. Close J, Ellis M, Hooper R, Glucksman E, Jackson S, Swift C. Prevention of falls in the elderly trial (PROFET): a randomised controlled trial. Lancet. 1999 Jan 9;353(9147):93-7.

5. Hendriks MR, Bleijlevens MH, van Haastregt JC, de Bruijn FH, Diederiks JP, Mulder WJ, et al. A multidisciplinary fall prevention program for elderly persons: a feasibility study. Geriatr Nurs. 2008 MayJun;29(3): 186-96.

6. Hendriks MR, van Haastregt JC, Diederiks JP, Evers SM, Crebolder HF, van Eijk JT. Effectiveness and costeffectiveness of a multidisciplinary intervention programme to prevent new falls and functional decline among elderly persons at risk: design of a replicated randomised controlled trial [ISRCTN64716113]. BMC Public Health. 2005 Jan 14;5:6.

7. Chang JT, Morton SC, Rubenstein LZ, Mojica WA, Maglione M, Suttorp MJ, et al. Interventions for the prevention of falls in older adults: systematic review and meta-analysis of randomised clinical trials. Bmj. 2004;328(7441):680.

8. Gillespie LD, Gillespie WJ, Robertson MC, Lamb SE, Cumming RG, Rowe BH. Interventions for preventing falls in elderly people. Cochrane Database Syst Rev. 2003(4):CD000340.

9. Gates S, Lamb SE, Fisher JD, Cooke MW, Carter YH. Multifactorial assessment and targeted intervention for preventing falls and injuries among older people in community and emergency care settings: systematic review and meta-analysis. Bmj. 2008 Jan 19;336(7636):130-3.

10. Campbell AJ, Robertson MC. Rethinking individual and community fall prevention strategies: a metaregression comparing single and multifactorial interventions. Age Ageing. 2007 Nov;36(6):656-62.

11. Weatherall M. Prevention of falls and fall-related fractures in community-dwelling older adults: a metaanalysis of estimates of effectiveness based on recent guidelines. Intern Med J. 2004 Mar;34(3):102-8.

12. Gillespie $L D$, Robertson MC, Gillespie WJ, Lamb SE, Gates S, Cumming RG, et al. Interventions for preventing falls in older people living in the community. Cochrane Database Syst Rev. 2009(2):CD007146. 
13. American Geriatrics Society BGS, and American Academy of Orthopaedic Surgeons Panel on Falls Prevention. Guideline for the prevention of falls in older persons. J Am Geriatr Soc. 2001;49(5):664-72.

14. Hendriks MR, Bleijlevens MH, van Haastregt JC, Crebolder HF, Diederiks JP, Evers SM, et al. Lack of effectiveness of a multidisciplinary fall-prevention program in elderly people at risk: a randomized, controlled trial. J Am Geriatr Soc. 2008 Aug;56(8):1390-7.

15. Jedeloo S, De Witte LP, Linssen BA, Schrijvers AJ. Client satisfaction with service delivery of assistive technology for outdoor mobility. Disabil Rehabil. 2002 Jul 10;24(10):550-7.

16. Ganz DA, Bao Y, Shekelle PG, Rubenstein LZ. Will my patient fall? JAMA. 2007 Jan 3;297(1):77-86.

17. Bleijlevens MH, Hendriks MR, van Haastregt JC, van Rossum E, Kempen GI, Diederiks JP, et al. Process factors explaining the ineffectiveness of a multidisciplinary fall prevention programme: a process evaluation. BMC Public Health. 2008 Sep 24;8(1):332.

18. O'Loughlin J, Robitaille Y, Bolvin J-F, Suissa S. Incidence of and risk factors for falls and injurious falls among the community-dwelling elderly. Am J Epidemiol. 1993;137:342-54.

19. Tinetti ME, Baker DI, McAvay G, Claus EB, Garrett P, Gottschalk M, et al. A multifactorial intervention to reduce the risk of falling among elderly people living in the community. N Eng/ J Med. 1994;331(13):821-7.

20. Kannus P, Sievanen H, Palvanen M, Jarvinen T, Parkkari J. Prevention of falls and consequent injuries in elderly people. Lancet. 2005 Nov 26;366(9500):1885-93.

21. Campbell AJ, Borrie MJ, Spears GF. Risk factors for falls in a community-based prospective study of people 70 years and older. J Gerontol. 1989;44(4):M112-7.

22. Davis JW, Ross $P D$, Nevitt MC, Wasnich RD. Risk factors for falls and for serious injuries on falling among older Japanese women in Hawaii. J Am Geriatr Soc. 1999;47:792-98.

23. Luukinen H, Koski K, Honkanen R, Kivela SL. Incidence of injury-causing falls among older adults by place of residence: a population-based study. J Am Geriatr Soc. 1995 Aug;43(8):871-6.

24. Nevitt MC, Cummings, S.R., Kidd, S., Black, D. Risk factor for recurrent nonsyncopal falls. JAMA. 1989;261:2663-8.

25. Rubenstein LZ. Falls in older people: epidemiology, risk factors and strategies for prevention. Age Ageing. 2006 Sep;35 Suppl 2:ii37-ii41.

26. Russell MA, Hill KD, Blackberry I, Day LL, Dharmage SC. Falls risk and functional decline in older fallers discharged directly from emergency departments. J Gerontol A Biol Sci Med Sci. 2006 Oct;61(10):1090-5.

27. Stalenhoef PA, Diederiks JP, Knottnerus JA, Kester AD, Crebolder HF. A risk model for the prediction of recurrent falls in community-dwelling elderly: a prospective cohort study. J Clin Epidemiol. 2002 Nov; 55(11):1088-94. 
28. Todd C, Skelton D. What are the main risk factors for falls among older people and what are the most effective interventions to prevent falls? Copenhagen: WHO Regional Office for Europe (Health Evidence Network report); 2004 March 2004. Report No.: E82552.

29. Brooks R. Euroqol:the current state of play. Health policy. $1996 \mathrm{Jul} ; 37(1): 53-72$.

30. Drummond MF, Sculpher MJ, Thorrance GW, O'Brien BJ, Stoddart GL. Methods for the economic evaluation of health care programmes. Third ed. New York: Oxford University Press; 2005.

31. Kempen GI, Miedema I, Ormel J, Molenaar W. The assessment of disability with the Groningen Activity Restriction Scale. Conceptual framework and psychometric properties. Soc Sci Med. 1996 Dec;43(11):1601-10.

32. Schuling J, de Haan R, Limburg M, Groenier KH. The Frenchay Activities Index. Assessment of functional status in stroke patients. Stroke. 1993 Aug;24(8):1173-7.

33. Gillespie LD, Gillespie WJ, Robertson MC, Lamb SE, Cumming RG, Rowe BH. Interventions for preventing falls in elderly people. Cochrane Database Syst Rev. 2009(2):CD000340.

34. Chou WC, Tinetti ME, King MB, Irwin K, Fortinsky RH. Perceptions of physicians on the barriers and facilitators to integrating fall risk evaluation and management into practice. J Gen Intern Med. 2006 Feb;21(2):117-22.

35. Fortinsky RH, lannuzzi-Sucich M, Baker DI, Gottschalk M, King MB, Brown CJ, et al. Fall-risk assessment and management in clinical practice: views from healthcare providers. J Am Geriatr Soc. 2004 Sep;52(9):1522-6.

36. Tinetti ME. Multifactorial fall-prevention strategies: time to retreat or advance. J Am Geriatr Soc. 2008 Aug;56(8):1563-5.

37. Tinetti ME, Gordon C, Sogolow E, Lapin P, Bradley EH. Fall-risk evaluation and management: challenges in adopting geriatric care practices. Gerontologist. 2006 Dec;46(6):717-25.

38. Yarnall KS, Pollak KI, Ostbye T, Krause KM, Michener JL. Primary care: is there enough time for prevention? Am J Public Health. 2003 Apr;93(4):635-41.

39. Lin MK, Marsteller JA, Shortell SM, Mendel P, Pearson M, Rosen M, et al. Motivation to change chronic illness care: results from a national evaluation of quality improvement collaboratives. Health Care Manage Rev. 2005 Apr-Jun;30(2):139-56.

40. Hill KD, Moore KJ, Dorevitch MI, Day LM. Effectiveness of falls clinics: an evaluation of outcomes and client adherence to recommended interventions. J Am Geriatr Soc. 2008 Apr;56(4):600-8.

41. Puisieux F, Pollez B, Deplanque D, Di Pompeo C, Pardessus V, Thevenon A, et al. Successes and setbacks of the falls consultation: report on the first 150 patients. Am J Phys Med Rehabil. 2001 Dec;80(12):909-15. 
42. Li F, Harmer P, Fisher KJ, McAuley E, Chaumeton N, Eckstrom E, et al. Tai Chi and fall reductions in older adults: a randomized controlled trial. J Gerontol A Biol Sci Med Sci. 2005 Feb;60(2):187-94.

43. Voukelatos A, Cumming RG, Lord SR, Rissel C. A randomized, controlled trial of tai chi for the prevention of falls: the Central Sydney tai chi trial. J Am Geriatr Soc. 2007 Aug;55(8):1185-91.

44. Wolf SL, Barnhart HX, Kutner NG, McNeely E, Coogler C, XU T. Reducing frailty and falls in older persons: an investigation of Tai Chi and computerized balance training. Atlanta FICSIT Group. Frailty and injuries: cooperative studies of intervention techniques. J Am Geriatr Soc. 1996 May;44(5):489-97.

45. Logghe IH, Zeeuwe PE, Verhagen AP, Wijnen-Sponselee RM, Willemsen SP, Bierma-Zeinstra SM, et al. Lack of effect of Tai Chi Chuan in preventing falls in elderly people living at home: a randomized clinical trial. J Am Geriatr Soc. 2009 Jan;57(1):70-5.

46. van Haastregt JC, Diederiks JP, van Rossum E, de Witte LP, Voorhoeve PM, Crebolder HF. Effects of a programme of multifactorial home visits on falls and mobility impairments in elderly people at risk: randomised controlled trial. Bmj. 2000;321(7267):994-8. 


\section{SUMMARY}

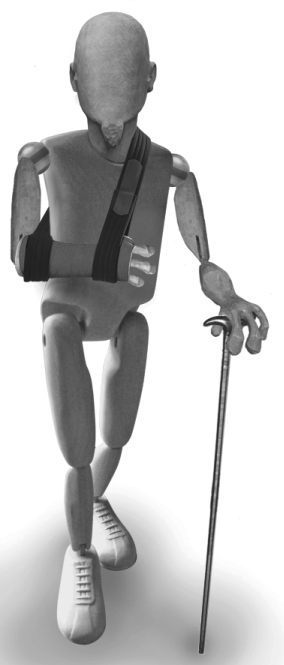


Falls severely threaten the health of older persons and can have a considerable impact on older persons and their relatives. Furthermore, falls place a heavy burden on the healthcare systems in many countries. About one third of community-dwelling older people aged 65 years and over fall at least once each year. Fallers who have sustained a fall are more likely to sustain further falls. In approximately $20 \%$ of falls, medical attention is needed, and about $10 \%$ of the falls experienced by people 65 years and over result in major injuries (e.g. fractures, joint distortions and dislocations, and severe head injuries). Persons who have sustained a fall show increased morbidity and healthcare utilization, resulting in increased healthcare costs. Even if a fall does not result in any kind of physical injury, it can have psychosocial consequences such as fear of falling and associated avoidance of activity.

In view of the impact and consequences of falls, it is important to develop fall prevention strategies. Due to the multifactorial origin of falls, a multifactorial fall prevention strategy, aimed at more than one risk factor simultaneously, seems to be beneficial. One such multi-faceted programme to prevent falls among elderly people attending the Accident and Emergency (A\&E) department after an injurious fall is the multidisciplinary programme developed by Close and colleagues. This programme, comprising a medical and occupational-therapy assessment, was highly effective in reducing the number of recurrent falls and associated injuries in London (United Kingdom). Since characteristics of the participants and healthcare setting appear to be critical, it cannot be automatically assumed that when a fall prevention programme is effective in a specific healthcare setting, this will be also the case in another healthcare setting. We therefore evaluated the effectiveness and feasibility of this intervention in a randomised controlled trial (RCT) in the Netherlands.

Chapter 1 comprises a general introduction to the research subject. It reports on the epidemiology and consequences of falls, causes and risk factors of falls, and the prevention of falls. The main objective of this thesis is to assess the effectiveness and feasibility of a medical and occupational-therapy programme to prevent falls and functional decline in elderly people who have sustained an injurious fall. In addition it explores opportunities to improve the prevention of injurious falls. The aims of this study are:

1. To assess the effectiveness of a multifactorial medical and occupational-therapy fall prevention programme among elderly people at risk, in terms of falls, functional decline, and a number of secondary outcomes. 
2. To assess the feasibility of this fall prevention programme for elderly people as well as the medical and paramedical practitioners who performed the assessments.

3. To assess the role of the occupational-therapy part of the fall prevention programme in preventing new falls and functional decline.

4. To assess whether it is possible to establish a classification of injurious fall types based on fall location and activity up to the moment of the fall.

5. To assess which risk factors predict new falls among a group of community-dwelling injurious fallers aged 65 years or over, in order to achieve a better selection of people at high risk for falling.

Chapter 2 presents the results of the two-group RCT assessing the effects of the multifactorial fall prevention programme. A total of 333 participants who were eligible to participate in this study were randomly allocated to the intervention $(n=166)$ and control $(n=167)$ groups. Results showed no significant favourable differences between the two groups in terms of falls, daily functioning, or any other secondary outcome after four and twelve months followup. Since our multidisciplinary fall prevention programme among community-dwelling older persons was not effective in preventing falls and functional decline in this Dutch healthcare setting, we do not recommend implementing the programme in its current form in the Netherlands.

Chapter 3 describes the process evaluation which was performed alongside the trial in order to assess the feasibility of our fall prevention programme. In addition, we tried to identify factors which might explain the lack of effectiveness of our programme. Data were collected from all participants allocated to the intervention group $(n=166)$ and the practitioners who performed the medical and occupational-therapy assessments $(n=8)$. This process evaluation revealed that $97 \%$ of the protocol items were carried out according to protocol. The number of referrals and recommendations ensuing from the medical assessment was relatively small. Participants' self-reported compliance as regards contacting their GPS to be informed of the recommendations and/or referrals was low to moderate. However, whenever the participants actually received the recommendations and/or referrals, selfreported compliance was reasonable to good. Both participants and practitioners judged the programme to be feasible and a large majority of participants reported they had benefited from the programme. The relatively low numbers of referrals and recommendations and the 
participants' disappointing compliance with the advice to contact their GPs are likely to be the main factors which are responsible for the lack of effectiveness of our programme.

Chapter 4 reports on the in-depth analysis of the occupational-therapy part of the multidisciplinary fall prevention programme, in order to gain insight into the contribution of the occupational-therapy part of the programme to the reduction in falls and functional decline. In contrast to the medical part of the programme, the occupational-therapy part resulted in a substantial number of referrals and recommendations which were directly communicated to the participants. The study population comprised 166 participants allocated to the intervention group of the RCT, two occupational therapists (OTs), and one official from each of the five participating municipalities. The occupational-therapy programme resulted in a total of 457 recommendations. Overall, about two thirds of all recommendations concerned advice about behaviour change, predominantly comprising recommendations to reduce risk. Sixty-five per cent of the recommendations regarding services and assistive devices were implemented. It took on average half a year to implement the recommended home modifications. To improve the occupational-therapy programme we suggest more rapid implementation of recommendations. Second, participants should be supported to achieve recommended changes. Furthermore, the occupational therapists should use theory-based techniques to stimulate behaviour change and use follow-up visits to promote maintenance of the desired behaviour.

Chapter 5 reports on a study which assessed whether it is possible to establish a classification of injurious fall types based on fall location and activity up to the moment of the fall. We carried out an exploratory, cross-sectional study to identify injurious fall types based on location of the fall and activity up to the moment of the fall. HOMALS (homogeneity analysis by means of alternating least squares) resulted in a classification of four injurious fall types: 1) Indoor falls related to lavatory visits (hall and bathroom); 2) Indoor falls during other activities of daily living; 3) Outdoor falls near the home during instrumental activities of daily living; 4) Outdoor falls away from home, occurring during walking, cycling, and shopping for groceries. There was no relationship between the four injurious fall types and severity of the consequences of the fall. However, there seems to be a difference in fall location and activity up to the moment of the fall between the younger and more active elderly persons, who still go outdoors, and the more frail older people who tend to stay indoors. Those persons who 
fell outdoors predominantly reported an extrinsic cause of their fall, whereas those persons who fell indoors reported an intrinsic cause. This could imply that persons who sustain an injurious fall should be offered a tailor-made intervention, based on the characteristics of the injurious fall typology.

Chapter 6 reports on an explorative study which assesses which risk factors predict new falls among a group of community-dwelling injurious fallers aged 65 years or over. The study population was derived from the trial assessing the effectiveness of the fall prevention programme. In this population ( $n=333$ ), a total of 15 variables were associated with a new fall $(p<0.05)$ and were subsequently entered into a multivariable logistic regression model. Recurrent falls in previous years, consequences of previous falls, ADL dependency, mobility, and age are most strongly associated with a new fall. All 15 possible predictors were entered simultaneously in a multivariable backward logistic regression model, resulting in a risk model comprising four predictors: age ( $\geq 79$ year), two or more falls in the previous year, experiencing more handicaps associated with previous fall(s) ( $F H I \geq 24)$, and having joint disorders. Based on the risk model we were able to develop a fall risk screening tool to determine what risk a patient runs for falling again. The area under the curve (AUC) of the receiver operating characteristic (ROC) curve was 0.77 (95\% Cl: 0.70-0.83), indicating that in $77 \%$ of the cases we were able to discriminate correctly between those who experienced a new fall and those who did not. Based on this study we recommend that healthcare workers in an A\&E department and GPs should primarily focus on four risk factors for sustaining a new fall among persons who have experienced an injurious fall. Based on the outcome of this risk assessment, healthcare workers can decide whether patients at sufficient risk will be offered an effective and feasible fall prevention programme.

Chapter 7 discusses the main findings of the studies described in the previous chapters (2-6). This is followed by a consideration of the current state of the art with regard to fall prevention research and some theoretical and methodological considerations. Finally, implications for practice and future research are given. Our multidisciplinary fall prevention programme comprising medical and occupational-therapy assessment did not have any favourable effects on falls and daily functioning among older persons of 65 years and over who had sustained an injurious fall. Nevertheless, it proved to be feasible and acceptable for both participants as well as the practitioners who performed the medical and occupational- 
therapy assessments. Considering the results of the studies described in this thesis, there remains a great need for effective fall prevention strategies. Therefore, fall prevention should remain a public health priority. We do not recommend the implementation of our fall prevention programme in its current form in regular care. It is of utmost importance to improve several aspects of the programme and assess whether these adaptations will result in greater effectiveness and efficiency. We recommend three major adjustments to improve the efficiency and effectiveness of our multidisciplinary fall prevention programme: a) adding an exercise component; b) improving the selection of the target population; and c) decreasing barriers to implementation. We recommend that in the Netherlands, fall prevention research should focus more on implementation research in order to gain additional insight into how to disseminate successful fall prevention strategies in regular healthcare. We need to create additional insight into factors explaining this lack of effectiveness. It is therefore important to include a detailed process-evaluation in fall prevention studies in the Netherlands and to pay more attention to the qualitative evaluation of implementation processes in the field of fall prevention in the Netherlands. 


\section{SAMENVATIING}

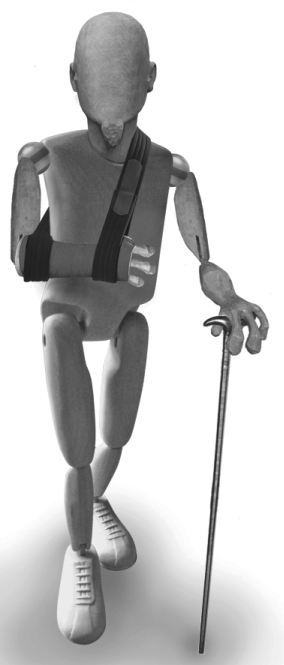


Valincidenten vormen een ernstige bedreiging voor de gezondheid van ouderen en hebben een aanzienlijke impact hebben op ouderen en hun familieleden. Bovendien vormen valincidenten in veel landen een zware last voor de gezondheidszorg. Ongeveer een derde van de zelfstandig wonende ouderen van 65 jaar en ouder valt tenminste één keer per jaar. Ouderen die reeds eerder gevallen zijn, hebben een verhoogd risico om opnieuw te vallen. In ongeveer 20 procent van de valincidenten is medische hulp noodzakelijk en ongeveer 10 procent van de valincidenten bij ouderen van 65 jaar en ouder resulteert in ernstige verwondingen (bijvoorbeeld breuken, verstuikingen, ontwrichtingen en ernstig hoofdletsel). Personen die zijn gevallen, hebben een verhoogde morbiditeit en een toename van het gezondheidszorggebruik, resulterend in hogere kosten voor de gezondheidszorg. Zelfs als een valincident niet leidt tot enige vorm van lichamelijk letsel, kan er sprake zijn van psychosociale gevolgen zoals valangst en het vermijden van activiteiten.

Om de impact en gevolgen van valincidenten te verminderen, is het belangrijk om valpreventie strategieën te ontwikkelen. Vanwege de multifactoriële oorsprong van valincidenten lijkt een multifactoriële valpreventie strategie, gericht op meerdere risicofactoren tegelijkertijd, aangewezen. Een van deze veelzijdige programma's om vallen te voorkomen bij ouderen, is het multidisciplinaire programma ontwikkeld door Close en collega's. Dat programma was gericht op ouderen die zich met verwondingen ten gevolge van een valincident hadden gemeld op de spoedeisende hulp (SEH). Dit programma, bestaande uit een medische en ergotherapeutische evaluatie, was zeer effectief in het verminderen van het aantal herhaalde valincidenten en daaraan gerelateerde verwondingen in Londen (Verenigd Koninkrijk). Het programma van Close werd toegepast op een specifieke groep ouderen woonachtig in Londen binnen de context van de gezondheidszorg in het centrum van deze metropool. Om die reden kan niet automatisch worden aangenomen dat dit in deze context effectief gebleken valpreventieprogramma, ook zonder meer effectief is in de context van de Nederlandse gezondheidszorg. Daarom hebben we het programma van Close aangepast aan de Nederlandse situatie en de effectiviteit en uitvoerbaarheid hiervan in de Nederlandse situatie opnieuw geëvalueerd door middel van een gerandomiseerd en gecontroleerd experimenteel onderzoek (RCT).

Hoofdstuk 1 omvat een algemene inleiding op het onderwerp en rapporteert over de epidemiologie, de gevolgen van valincidenten, oorzaken en risicofactoren van 
valincidenten en het voorkomen van valincidenten. Het belangrijkste doel van dit proefschrift is het vaststellen van de effectiviteit en uitvoerbaarheid van een medisch en ergotherapeutisch programma, gericht op het voorkomen van valincidenten en functionele achteruitgang bij ouderen, die een valincident met letsel hebben doorgemaakt en daarvoor de SEH of Huisartspost van een ziekenhuis hebben bezocht. Daarnaast worden de mogelijkheden onderzocht om de preventie van valincidenten met letsel te verbeteren. De doelstellingen van deze studie zijn:

1. Evaluatie van de effectiviteit van een multifactorieel medisch en ergotherapeutisch valpreventieprogramma onder ouderen met een verhoogd valrisico in termen van vallen, functionele achteruitgang en een aantal secundaire uitkomsten.

2. Evaluatie van de uitvoerbaarheid van dit valpreventieprogramma voor zowel ouderen, als ook de (para)medici die de evaluaties uitvoeren.

3. Evaluatie van de rol van het ergotherapeutische deel van valpreventieprogramma om nieuwe valincidenten en functionele achteruitgang te voorkomen.

4. Onderzoek naar de mogelijkheden om een classificatie van valincidenten met letsel vast te stellen op basis van vallocatie en de activiteit die werd ondernomen op het moment van het valincident.

5. Onderzoek naar welke risicofactoren nieuwe valincidenten voorspellen in een groep van zelfstandig wonende ouderen van 65 jaar of ouder die een valincident met letsel hebben doorgemaakt, met het oog op een betere selectie van mensen met een verhoogd valrisico.

Hoofdstuk 2 presenteert de resultaten van een gerandomiseerd experimenteel onderzoek (RCT) met twee groepen naar de effecten van een multifactorieel valpreventieprogramma. De geselecteerde deelnemers $(n=333)$ werden willekeurig aan een interventiegroep $(n=166)$ of aan een controlegroep $(n=167)$ toegewezen. Noch na vier noch na twaalf maanden follow-up waren er significante verschillen te zien tussen de twee groepen ten aanzien van het aantal valincidenten, het dagelijks functioneren, en de overige uitkomstmaten. Om deze reden adviseren wij dit programma in zijn huidige vorm niet in Nederland te implementeren.

Hoofdstuk 3 beschrijft de procesevaluatie die parallel aan de effectevaluatie werd uitgevoerd om de uitvoerbaarheid van ons valpreventieprogramma te onderzoeken. Daarnaast hebben we geprobeerd factoren te identificeren die het gebrek aan effectiviteit zouden kunnen verklaren. Gegevens werden verzameld bij alle deelnemers van de interventie groep $(n=166)$ en 
uitvoerders van de medisch en ergotherapeutische evaluatie $(n=8)$. Uit de analyse bleek dat $97 \%$ van de protocol items werden uitgevoerd volgens protocol. Het aantal verwijzingen en aanbevelingen op grond van de medische evaluatie was gering. Het aantal deelnemers dat het advies opvolgde om contact op te nemen met de huisarts om geïnformeerd te worden over de aanbevelingen en/of verwijzingen, was gering tot matig. Dat advies werd naar eigen zeggen wel redelijk tot goed opgevolgd, door de groep deelnemers die daadwerkelijk aanbevelingen en/of verwijzingen ontvingen. Zowel de deelnemers, als de uitvoerders van de evaluaties beoordeelden het programma als uitvoerbaar en een grote meerderheid van de deelnemers rapporteerde baat bij het programma. Het relatief lage aantal verwijzingen en aanbevelingen en geringe aantal deelnemers dat het advies om contact op te nemen met de huisarts opvolgde, zijn waarschijnlijk verantwoordelijk zijn voor de ineffectiviteit van het programma.

Hoofdstuk 4 beschrijft een analyse van het ergotherapeutisch onderdeel van het multidisciplinair valpreventieprogramma, met als doel het verkrijgen van inzicht in de specifieke bijdrage van dit deel aan de mogelijke vermindering van valincidenten en functionele achteruitgang. In tegenstelling tot het medische deel van het programma, resulteerde het ergotherapeutisch deel wel in een groot aantal verwijzingen en aanbevelingen, die rechtstreeks werden meegedeeld aan de deelnemers. De populatie bestond uit 166 deelnemers die waren toegewezen aan de interventie groep van het RCT, twee ergotherapeuten, en verder een ambtenaar van elk van de vijf bij het onderzoek betrokken gemeentelijke Wet Voorzieningen Gehandicapten (WVG) afdelingen. Dit deel van het programma resulteerde in 457 aanbevelingen. Ongeveer twee derde van alle aanbevelingen waren adviezen met betrekking tot de verandering van gedrag, voornamelijk bestaande uit aanbevelingen om risicogedrag te verminderen. Uit de zelfrapportage bleek dat vijfenzestig procent van de aanbevelingen met betrekking tot diensten en hulpmiddelen werd opgevolgd. Het duurde gemiddeld een half jaar om de aanbevolen woningaanpassingen te implementeren. Ter verbetering van het ergotherapeutisch programma wordt een snellere uitvoering van de aanbevelingen aangeraden. Ten tweede dienen de deelnemers beter ondersteund te worden om aanbevolen veranderingen in het dagelijks leven in te passen. Bovendien dienen de ergotherapeuten gebruik te maken van technieken om gedragsverandering te stimuleren en follow-up bezoeken uit te voeren, om behoud van het gewenste gedrag te realiseren. 
Hoofdstuk 5 rapporteert over een studie waarin werd onderzocht of het mogelijk is om een classificatie van valincidenten met letsel, vast te stellen op basis van vallocatie en de activiteit op het moment van het valincident. Het betreft een explorerend, cross-sectioneel onderzoek. De statistische techniek HOMALS (homogeniteit analyse door middel van alternerende kleinste kwadraten) resulteerde in een indeling van vier typen valincidenten met letsel: 1) Valincidenten binnenshuis gerelateerd aan toiletbezoek (hal en badkamer, 2) Valincidenten binnenshuis tijdens andere activiteiten van het dagelijks leven, 3) Valincidenten buitenshuis in de buurt van de eigen woning tijdens instrumentele activiteiten van het dagelijks leven, 4) Valincidenten binnenshuis niet in de buurt van de eigen woning, zoals tijdens wandelen, fietsen en/of boodschappen doen. $\mathrm{Er}$ is geen relatie gevonden tussen de vier typen valincidenten met letsel en de ernst van de gevolgen van het valincident. Echter, er lijkt een verschil te zijn wat betreft vallocatie en activiteit op het moment van het valincident tussen de jongere en meer actieve ouderen, die nog altijd buitenshuis komen, aan de ene kant en de meer kwetsbare ouderen met de neiging om binnenshuis te blijven. De mensen die buitenshuis zijn gevallen, rapporteerden voornamelijk een extrinsieke oorzaak van hun val, terwijl degenen die binnenshuis vielen een intrinsieke oorzaak aangaven. Dit zou kunnen betekenen dat personen met een valincident met letsel, op maat gemaakte interventies moeten worden aangeboden, gebaseerd op de kenmerken van de typologie.

Hoofdstuk 6 doet verslag van een explorerende prospectieve studie, waarin wordt onderzocht welke risicofactoren nieuwe valincidenten voorspellen in een groep van zelfstandig wonende ouderen van 65 jaar of ouder, die een valincident met letsel hebben doorgemaakt. De onderzoekspopulatie bestaat uit de totale groep deelnemers aan het RCT. In deze populatie $(n=333)$ waren 15 variabelen gerelateerd aan een nieuw valincident $(p<0,05)$ en deze werden vervolgens ingevoerd in een multivariabel logistisch regressiemodel. Herhaalde valincidenten in voorgaande jaren, de gevolgen van een eerdere val, algemene dagelijks levensverrichtingen (ADL) afhankelijkheid, mobiliteit, en leeftijd zijn het sterkst gerelateerd aan een nieuw valincident. Alle 15 mogelijke voorspellers werden tegelijkertijd opgenomen in een multivariabel logistisch regressie model met achterwaartse selectie procedure, wat resulteerde in een risicomodel bestaande uit vier voorspellers: leeftijd ( $\geq 79$ jaar), twee of meer valincidenten in het voorgaande jaar, ervaren nadelen gerelateerd aan eerdere valincidenten ( $F H I \geq 24)$, en het lijden aan gewrichtsaandoeningen. Op basis van dit model konden we een valrisico screenings instrument ontwikkelen, om te bepalen in welke mate 
een patiënt risico loopt om opnieuw te vallen. Het gebied onder de curve (AUC) van de 'Receiver Operating Characteristic' (ROC) curve was 0,77 (95\% Cl: 0.70-0.83), waaruit blijkt dat in $77 \%$ van de gevallen een correct onderscheid gemaakt kan worden tussen degenen die opnieuw vielen en degenen die niet opnieuw vielen. Op basis hiervan wordt aanbevolen dat behandelaars op de spoedeisende hulp en huisartsen zich primair dienen te richten op deze vier risicofactoren. Op grond daarvan kunnen behandelaars dan beslissen of patiënten een valpreventieprogramma wordt aangeboden.

Hoofdstuk 7 bespreekt de belangrijkste bevindingen van de studies beschreven in de vorige hoofdstukken (2-6). Dit wordt gevolgd door een beschouwing van de huidige stand van zaken met betrekking tot valpreventieonderzoek en een aantal theoretische en methodologische overwegingen. Ten slotte worden implicaties voor de praktijk en toekomstig onderzoek aangegeven. Ons multidisciplinair valpreventieprogramma had geen effect op vallen en het dagelijks functioneren van oudere personen van 65 jaar en ouder die een valincident met letsel hadden doorgemaakt. Wel bleek het programma uitvoerbaar en aanvaardbaar voor zowel de deelnemers als de uitvoerders van de medische en ergotherapeutische evaluaties.

De resultaten van ons onderzoek maken duidelijk dat er nog steeds een grote behoefte bestaat aan effectieve strategieën voor valpreventie. Daarom moet valpreventie hoog op de volksgezondheid agenda blijven staan. Het is van groot belang dat verschillende onderdelen van het programma worden verbeterd en dat geëvalueerd wordt of deze aanpassingen wel leiden tot de beoogde grotere effectiviteit en efficiency. Drie belangrijke aanpassingen bevelen we aan om de efficiëntie en effectiviteit van ons valpreventieprogramma te verbeteren: a) toevoeging van een "exercise" component (oefenprogramma); b) verbetering van de selectie van de doelgroep, en c) vermindering van belemmeringen bij de implementatie.

Met betrekking tot valpreventie onderzoek in Nederland moet meer nadruk gelegd worden op implementatie onderzoek, om zodoende meer inzicht te krijgen in de wijze waarop succesvolle valpreventie strategieën in de reguliere gezondheidszorg kunnen worden toegepast. We moeten meer inzicht verkrijgen in de factoren die het gebrek aan effectiviteit kunnen verklaren. Het is daarom belangrijk om een gedetailleerde procesevaluatie toe te voegen aan valpreventie studies en om meer aandacht te besteden aan de kwalitatieve evaluatie van implementatie processen op het gebied van valpreventie. 


\section{CO-AUTHORS \& AFFILIATIONS}

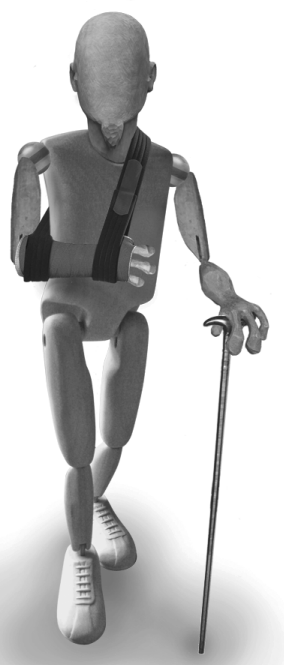


Harry FJM Crebolder, MD, PhD

Maastricht University, Faculty of Health, Medicine and Life Sciences, Department of General Practice, School for Public Health and Primary Care (Caphri), Maastricht, The Netherlands

Joseph PM Diederiks, PhD

School for Public Health and Primary Care (Caphri), Maastricht, The Netherlands

Jacques ThM van Eijk, PhD

Maastricht University, Faculty of Health, Medicine and Life Sciences, Department of Social Medicine, School for Public Health and Primary Care (Caphri), Maastricht, The Netherlands

Silvia MAA Evers, PhD

Maastricht University, Faculty of Health, Medicine and Life Sciences, Department of Health Organization, Policy and Economics, School for Public Health and Primary Care (Caphri), Maastricht, The Netherlands

Jolanda CM van Haastregt, PhD

Maastricht University, Faculty of Health, Medicine and Life Sciences, Department of Health Care and Nursing Science, School for Public Health and Primary Care (Caphri), Maastricht, The Netherlands

Marike RC Hendriks, PhD

Maastricht University, Faculty of Health, Medicine and Life Sciences, Department of Health Organization, Policy and Economics and Department of Clinical Epidemiology and Medical Technology Assessment, School for Public Health and Primary Care (Caphri), Maastricht, The Netherlands

Gertrudis IJM Kempen, PhD

Maastricht University, Faculty of Health, Medicine and Life Sciences, Department of Health Care and Nursing Science, School for Public Health and Primary Care (Caphri), Maastricht, The Netherlands 
Wubbo J Mulder, MD, PhD

Maastricht University Hospital, Department of Internal Medicine, Division Medicine and Geriatrics, Maastricht, the Netherlands

Erik van Rossum, PhD

Zuyd University of Applied Sciences, Research Centre on Autonomy and Participation of chronically ill patients, Heerlen and Maastricht University, Faculty of Health, Medicine and Life Sciences, Department of Health Care and Nursing Science, School for Public Health and Primary Care (Caphri), Maastricht, The Netherlands

Joop M Ruijgrok, MD, PhD

Maastricht University Hospital, Department of Internal Medicine, Division Medicine and Geriatrics, Maastricht, the Netherlands

Paul A Stalenhoef, MD, PhD

Maastricht University, Faculty of Health, Medicine and Life Sciences, Department of General Practice, School for Public Health and Primary Care (Caphri), Maastricht, The Netherlands 

PUBLICATIONS

PUBLICATIONS

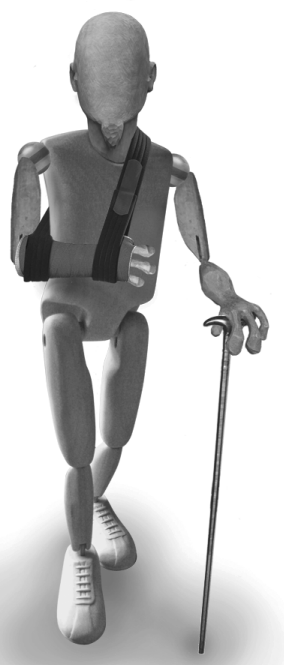




\section{PUBLICATIONS}

Gulpers MJM, Bleijlevens MHC, van Rossum E, Capezuti E, Hamers JPH. Belt restraint reduction in nursing homes: Design of a quasi-experimental study. BMC Geriatrics. 2010; In press.

Bleijlevens MH, Hendriks MR, Van Haastregt JC, Crebolder HF, Van Eijk JT. Lessons learned from a multidisciplinary fall-prevention programme: The occupational-therapy part. Scand J Occup Ther. 2009 Dec 14.

Hamers JPH, Gulpers MJM, Bleijlevens M, Huizing AR, Scherder EJ, Houweling H, Van Rossum E. Het reduceren van vrijheidsbeperking in verpleeghuizen. Tijdschrift voor Ouderengeneeskunde. 2009;5: 156-9.

Bleijlevens MH, Hendriks MR, van Haastregt JC, van Rossum E, Kempen GI, Diederiks JP, et al. Process factors explaining the ineffectiveness of a multidisciplinary fall prevention programme: a process evaluation. BMC Public Health. 2008;8:332.

Hendriks MR, Bleijlevens MH, van Haastregt JC, Crebolder HF, Diederiks JP, Evers SM, et al. Lack of effectiveness of a multidisciplinary fall-prevention program in elderly people at risk: a randomized, controlled trial. J Am Geriatr Soc. 2008 Aug;56(8):1390-7.

Hendriks MR, Bleijlevens MH, van Haastregt JC, de Bruijn FH, Diederiks JP, Mulder WJ, et al. A multidisciplinary fall prevention program for elderly persons: a feasibility study. Geriatr Nurs. 2008 May-Jun;29(3):186-96.

Hendriks MR, Evers SM, Bleijlevens MH, van Haastregt JC, Crebolder HF, van Eijk JT. Costeffectiveness of a multidisciplinary fall prevention program in community-dwelling elderly people: a randomized controlled trial (ISRCTN 64716113). Int J Technol Assess Health Care. 2008 Spring;24(2):193-202. 


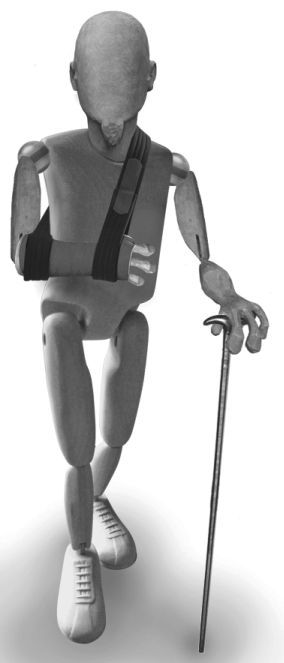


En dan is de tijd aangebroken om los te laten. lets loslaten, de regie uit handen geven, is voor mij allerminst makkelijk. Als rasechte controlefreak heb ik in de afgelopen 5 jaar geprobeerd zoveel mogelijk de controle te houden over datgene wat uiteindelijk geleid heeft tot dit proefschrift. Uiteraard was me dat zonder de kennis, kunde en ondersteuning van u, jou, jullie nooit gelukt, waarvoor mijn welgemeend dank je wel! Een aantal mensen wil ik echter apart noemen, omdat zij op een speciale wijze hebben bijgedragen aan het tot stand komen van dit proefschrift.

Op de eerste plaats wil ik alle deelnemers aan het onderzoek bedanken, bij de deelnemers beter bekend als "Interval". Zonder uw bereidwillige deelname, geen gegevens, geen proefschrift. Dit geldt uiteraard ook voor de medewerking van alle bij dit onderzoek betrokken organisaties (azM, Regionaal Indicatie Orgaan Maastricht, gemeente Maastricht, gemeente Valkenburg, gemeente Meerssen, gemeente Margraten, gemeente Eijsden en het Memic (met name Jack Berben, Annemie Mordant, Hilde Sielhorst en Marlene Ronner)).

Dan mijn promotoren en copromotor Jacques van Eijk, Harry Crebolder en Jolanda van Haastregt. Met al jullie capaciteiten vormen jullie een uitgebalanceerd begeleidingsteam, samen altijd richting het einddoel sturend. Ik heb me vanaf dag één bij jullie op mijn gemak gevoeld. Dank voor jullie vertrouwen in mijn kunnen, ook op de momenten dat ik daar zelf aan twijfelde. Jacques, jouw rol als "advocaat van de duivel" speelde je met verve. Je kritische opmerkingen gaven stof tot nadenken, resulterend in een verbeterde versie van een artikel. Ook dank voor je niet aflatende interesse in zowel de voortgang, als ook zaken die mij naast het werk bezig hielden. Harry, ik hoop nog veel mails van jou te ontvangen met de afsluitende zin: "Goede moed". Dank voor je altijd positieve insteek en kritische opmerkingen. Jij stond misschien wat meer op de achtergrond, maar was altijd meteen beschikbaar als ik met je wilde overleggen. Ook de snelheid waarmee je stukken van commentaar voorzag heb ik gewaardeerd: " En nu de snelheid erin houden"! Jolanda, auteur van de "oranje bijbel". Ik bewonder je manier van commentaar geven. Duidelijk, helder, maar bovenal opbouwend. Jij kunt moeilijke zinsconstructies als geen ander vereenvoudigen. Dank voor al je suggesties en je kritische houding. Ik heb enorm veel van je geleerd. Ondanks je overvolle agenda was je altijd beschikbaar om over van alles en nog wat van gedachten te wisselen. Ik waardeer dat nog steeds. 
Alle coauteurs en/of leden van de Interval projectgroep wil ik van harte bedanken voor alle ondersteuning. Ruud Kempen, Luc de Witte, Paul Stalenhoef, Peter Brink, Wubbo Mulder, Joop Ruygrok en in het bijzonder Erik van Rossum en Jos Diederiks. Erik, sinds het begin van mijn academische carrière kan ik op jou rekenen. Jij hebt me als mogelijke kandidaat voor het Interval project naar voren geschoven. Het lijkt wel of we met een touwtje aan elkaar vastzitten. Telkens weer komt het tot een voor mij uitermate leerzame en prettige samenwerking. Jos, jij hebt mij benaderd voor een sollicitatiegesprek op aanraden van Erik. Dank dat je destijds de telefoon gepakt hebt. Als begeleider van het eerste uur wil ik je bedanken voor je inzet. Jouw statistische kennis en kunde heb ik regelmatig nodig gehad. Alle mensen die het valpreventieprogramma in de praktijk hebben uitgevoerd: Ludo Scheres, Ron Warnier, Tanja de Bie, Charlotte Mom, Evelien Cratzborn en nogmaals Wubbo en Joop. Dat dit programma hoge ogen gooit wat betreft uitvoerbaarheid is mede aan jullie te danken!

De leden van de beoordelingscommissie onder voorzitterschap van Prof. dr. G.J. Dinant wil ik bedanken voor het beoordelen van het manuscript.

Al mijn (oud) collega's bij Medische Sociologie, Praxis für physiotherapie Houben Kemperink, Sociale Geneeskunde, Stichting Land van Horne, Verpleging en Verzorging en Sevagram. Hartelijk dank voor jullie interesse in mijn onderzoeksactiviteiten, jullie ontelbare adviezen en jullie gezelligheid. Dit laatste is vooral van toepassing op Mieke, Liesbeth, Gonnie, Vivian, Ramon, Math en Marike, allemaal kamergenoten die ik tijdens mijn vele verhuizingen van de afgelopen 5 jaar binnen de UNS40 West beter heb leren kennen.

Lieve vrienden, dank voor de broodnodige ontspanning middels prachtige vakanties, weekendjes weg, heerlijke etentjes, het bezoeken van voetbalwedstrijden, het bezoeken van festivals, geweldige (hoedjes)feestjes, carnaval (vooral in 2009: wat woar dat sjun!), het organiseren van het mooiste criterium van Nederland (de Profronde Heerlen) en het behalen van de wereldtitel op het Wereld Muziek Concours (WMC). Ik ben trots op jullie en op onze vriendschap. 
Pat, ik waardeer de manier waarop jij invulling geeft aan onze vriendschap. Ik ken niemand die meer voor een ander klaar staat dan jij. Vele mooie dingen hebben we al samen mogen beleven, vele zullen er ongetwijfeld nog volgen. Het betekent veel voor me dat jij op 16 april naast me staat. Marike, samen hebben we het Interval project nu tot een goed einde gebracht. Onze intensieve samenwerking mis ik regelmatig, vooral de manier waarop wij elkaar aanvullen. Jij sprak ooit over de eigen taal die we ontwikkeld hebben. Die bestaat echt! Dank je wel dat jij aan de andere kant naast me komt staan. Pat en Marike, ik ben er trots op dat jullie mijn paranimfen zijn.

Mijn ouders en schoonouders wil ik bedanken voor de goede zorgen tijdens mijn gehele promotietraject. Jullie stonden altijd voor me klaar en hebben mij en Jill heel wat werk uit handen genomen. Pap en Mam, toch wil ik jullie nog bijzonder bedanken. Jullie vormen het fundament onder alles wat ik doe. Jullie hebben mij altijd het vertrouwen geschonken en de ruimte gegeven om mijn eigen weg te gaan. Jullie liefde en oprechte belangstelling is mij veel waard.

Lieve Jill, het feit dat jij er altijd voor me bent, zorgt ervoor dat ik kan zijn wie ik ben. Jouw steun en onvoorwaardelijke liefde wil ik nooit meer missen. 


\section{CURRICULUM VITAE}

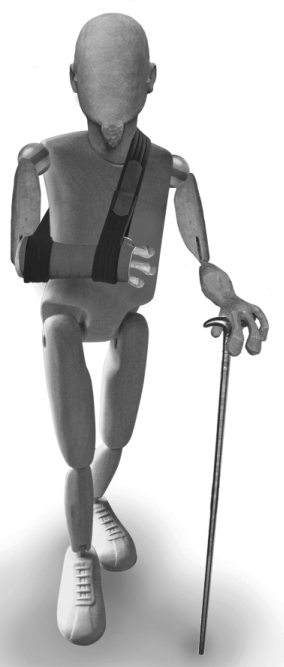



Michel Bleijlevens was born in 1978 in Heerlen. He attended secondary school (VWO) at the Katholiek Gymnasium Rolduc in Kerkrade. After his graduation in 1997 he studied Health Sciences at Maastricht University (Maastricht) and Physiotherapy at Zuyd University of Applied Sciences (Heerlen). He completed both his master degree in Movement Science and bachelor degree in Physiotherapy in 2004. In November 2004 he started as a research assistant in a fall prevention project at the Faculty of Health, Medicine and Life Sciences, and School for Public Health and Primary Care (Caphri), of Maastricht University. In March 2005 he was appointed as a PhD researcher on this project which included a randomised controlled trial to assess the effects and feasibility of a multidisciplinary fall prevention programme aimed at community-dwelling elderly people at risk for falling. Since October 2008 he is affiliated with the same faculty and research school as a research fellow. As such, he is involved in a quasi-experimental study on the reduction of belt use in nursing homes (EXBELT). His current research activities are part of the CAPHRI research programme "Innovations in the care for the elderly". Since 2004 he combined his (PhD) research with clinical work as physiotherapist in various health care settings (primary care in KerpenSindorf, Germany, and nursing home care in Weert). At present he still combines his research activities with clinical work. He is physiotherapist and project manager of an innovation programme aimed at physical activities of nursing home residents at Sevagram in Heerlen. 
